\title{
Standard Propagation Channel Models for MIMO Communication Systems
}

\author{
Agbotiname Lucky Imoize $\mathbb{D}^{\mathbb{D}},{ }^{1,2}$ Augustus Ehiremen Ibhaze $\mathbb{D}^{2},{ }^{2}$ Aderemi A. Atayero $\mathbb{D}^{3}{ }^{3}$ \\ and K. V. N. Kavitha ${ }^{4}{ }^{4}$ \\ ${ }^{1}$ Wireless@VT, Bradley Department of Electrical and Computer Engineering, Virginia Polytechnic Institute and State University, \\ Blacksburg, 24061 VA, USA \\ ${ }^{2}$ Department of Electrical and Electronics Engineering, University of Lagos, Akoka, 100213 Lagos, Nigeria \\ ${ }^{3}$ Department of Electrical and Information Engineering, Covenant University, Ota, 112233 Ogun State, Nigeria \\ ${ }^{4}$ Department of Communication Engineering, School of Electronics Engineering (SENSE), Vellore Institute of Technology, Vellore, \\ 632014 Tamil Nadu, India
}

Correspondence should be addressed to Agbotiname Lucky Imoize; aimoize@vt.edu

Received 30 September 2020; Revised 14 January 2021; Accepted 19 January 2021; Published 16 February 2021

Academic Editor: Daniele Pinchera

Copyright (c) 2021 Agbotiname Lucky Imoize et al. This is an open access article distributed under the Creative Commons Attribution License, which permits unrestricted use, distribution, and reproduction in any medium, provided the original work is properly cited.

\begin{abstract}
The field of wireless communication networks has witnessed a dramatic change over the last decade due to sophisticated technologies deployed to satisfy various demands peculiar to different data-intensive wireless applications. Consequently, this has led to the aggressive use of the available propagation channels to fulfill the minimum quality of service (QoS) requirement. A major barometer used to gauge the performance of a wireless communication system is the spectral efficiency (SE) of its communication channels. A key technology used to improve SE substantially is the multiple input multiple output (MIMO) technique. This article presents a detailed survey of MIMO channel models in wireless communication systems. First, we present the general MIMO channel model and identified three major MIMO channel models, viz., the physical, analytical, and standardized models. The physical models describe the MIMO channel using physical parameters. The analytical models show the statistical features of the MIMO channel with respect to the measured data. The standardized models provide a unified framework for modern radio propagation architecture, advanced signal processing, and cutting-edge multiple access techniques. Additionally, we examined the strengths and limitations of the existing channel models and discussed model design, development, parameterization, implementation, and validation. Finally, we present the recent 3GPP-based 3D channel model, the transitioning from $2 \mathrm{D}$ to $3 \mathrm{D}$ channel modeling, discuss open issues, and highlight vital lessons learned for future research exploration in MIMO communication systems.
\end{abstract}

\section{Introduction}

Over the last decade, the use of wireless communication devices and applications has increased exponentially. The widespread adoption of these devices places a stringent requirement on the existing wireless network infrastructure to be strengthened in terms of robustness, capacity, and coverage to meet the rapidly growing mobile services demands. Currently, wireless network service improvements require the aggressive deployment of dense access points to increase the spectral efficiency (SE) and energy efficiency (EE) $[1,2]$.
To achieve high gains in spectral, energy, and hardware efficiency in wireless communication systems, the deployment of multiple input multiple output (MIMO) technology presents enormous potentials as a leading candidate. Toward this end, the concept of cellular networks and ways to improve spectral efficiency with practical examples are proposed in [3]. The monograph covers critical areas such as spatial signal processing, channel estimation, power, and spatial resource allocation. It also emphasized that massive MIMO can deliver remarkable improvements in spectral and energy efficiencies. Also, several research works [4-7] 
have proposed the deployment of multiple antennas, also known as MIMO, as a candidate technology. This is because MIMO technology has promising capabilities to achieving the desired high data rates and very high network reliability, thereby enhancing the quality of service (QoS) for mobile subscribers to sustain their vast data-hungry applications.

Rappaport [8] presents an overview of the radio propagation channel, and an introduction to the wireless channel model and channel statistics is given in [9]. Similarly, a detailed description of propagation channel modeling is discussed in [10], and a thorough review of propagation measurements, parameterization, and model validation is presented in $[11,12]$. In related reports, an overview of MIMO channel propagation models, including an extensive description of modern signal processing techniques for single-user and multiuser systems, is presented in [13, 14]. Also, the fundamentals of massive MIMO have been presented in [15]. Furthermore, the performance limitations of wireless networks were outlined, and the various ways of improving the efficiency of wireless networks were provided in [15]. Also, the techniques and methods of massive MIMO channel modeling with practical and analytical examples were presented. In a related work [16], near field communication (NFC) system channel models were introduced, and a modified model was proposed for application in magnetoinductive communications. The authors demonstrated that the performance of the modified model showed remarkable improvements over the models reported in [17-19]. However, the authors observed that more efficient channel models are required for real-time near field communications.

In recent surveys [20-22], the focus is specifically on $5 \mathrm{G}$, and there is no adequate information about how earlier channel models contributed to the rapid development and deployment of modern channel models, especially for 5G and beyond [23]. In particular, 5G channel measurements and models were reported in [20], the usage scenarios and traffic models in 5G were surveyed in [21], and MIMO localization in $5 \mathrm{G}$ was explored in [22]. In the literature [20-22], it was not adequately emphasized how the knowledge of earlier channel models has influenced the design and development of the current $5 \mathrm{G}$ channel models like the ones reported in [24-26]. Besides, the authors in [20] seem to have overlooked the possibility of applying the $5 \mathrm{G}$ channel models to other environments different from the geographical locations for which they have been designed. Though wireless channel models provide valuable information required for efficient network planning, deployment, and optimization [27], but channel models developed in the past tend to focus only on the modeling aspects that were of key interests at the time. Therefore, the need to explore how the earliest wireless channel models influence the design and development of the emerging MIMO channel models becomes imperative.

This survey attempts to bridge the gap between the conventional and the recently developed channel models by first classifying them into different categories and exploring their interrelationships and enormous potentials. Second, we try to determine why it is quite challenging to develop a generalized channel model, which accurately predicts the path loss, considering different environmental conditions. Third, we explore the literature to examine future channel models with adaptable features for different wireless propagation environments, including several dynamic environmental factors missing in the existing channel models. This survey is set out to explore whether a single channel model fits well to all environments' categorization. The survey also is aimed at knowing whether adequate knowledge of the existing channel models would be indispensable to develop emerging or future learning-based channel models. We also need to know whether the envisioned artificial intelligence or machine learning-based models would be independent of the current channel models.

Further to this, the survey looks at the propagation channel model's testing procedures and validation criteria. The survey also examines the need for actual field measurements to validate customized channel models and assess the impact of the measurements equipment on the developed channel model. The study examines the measurement equipment reported in earlier literature and assesses their capabilities and limitations. Furthermore, the investigation takes an exploratory view of the existing MIMO channel models' strengths and limitations. Last, we present the open research issues and highlight vital take-away lessons for informed decision making by the wireless research community.

The remaining part of this paper is structured as follows. Section 2 presents the general MIMO channel model and its core requirements, multilink channel sounding techniques, MIMO technology classification, and MIMO channel modeling. Section 3 focuses on the physical models comprising of deterministic and stochastic channel models. Analytical models consisting of the correlation-based, propagationmotivated models, and channel models based on space-time coding are reported in Section 4 . Section 5 provides an overview of the standardized channel models, with particular reference to the 3GPP spatial channel models, the COST 2100 model, WINNER II/+ channel models, and the IEEE standards. Section 6 focuses on 3D channel modeling, emphasizing 3D channel models' elevation characteristics and the transitioning from the conventional $2 \mathrm{D}$ to $3 \mathrm{D}$ channel modeling. Open issues for future research are covered in Section 7, lessons learned are briefly outlined in Section 8, and Section 9 gives the conclusion to the paper.

\section{The General MIMO Channel Model}

The basic MIMO channel model and its core requirements, multilink channel sounding techniques, MIMO technology classification, and MIMO channel modeling are presented in this section. First, the generalized MIMO channel model and MIMO channel modeling techniques are introduced. Second, the concept of multilink channel sounders is presented briefly. This section also covers the single-sounder sequential measurements, single-sounder multinode measurements, and multisounder measurements. Further to this, we highlight the basic classification of MIMO channel models, point-to-point, multiuser MIMO, and massive MIMO, and provided a concise description of the multiuser MIMO for the uplink and downlink scenarios. 


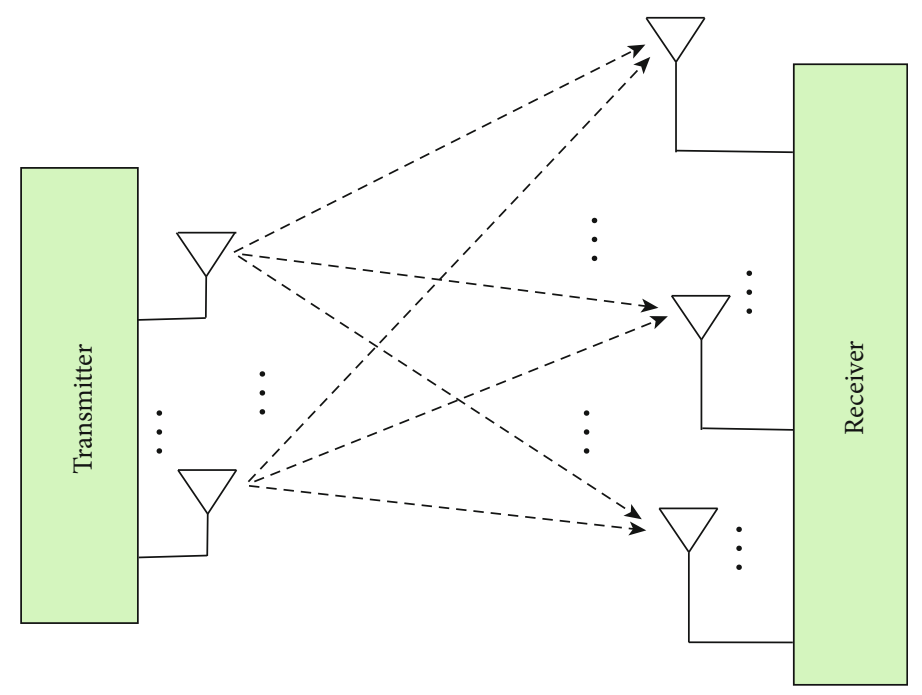

FIgURE 1: A basic MIMO channel showing multiple transmit and receive antennas.

2.1. Basic MIMO Channel Model. The traditional single antenna communication system lacks the required spatial degree of freedom provided by multiple antennas. In MIMO communication systems, the presence of multiple antennas can be of great advantage. The spatial degree of freedom provided by these antennas can be appropriately harnessed to boost the capacity and expand the system's coverage through optimal scheduling of multiple users to simultaneously share the spatial channel. As illustrated in Figure 1, the channel gives useful information about transmitting and receiving antennas in a simple MIMO channel. Considering the case of an $n \times m$ MIMO channel system, where $m$ and $n$ represent the respective numbers of transmitting and receiving antennas, the linear time-variant MIMO channel could be modeled as shown in (1), using an $n \times m$ channel matrix [28].

$H(t, \tau)=\left[\begin{array}{ccccc}h_{11}(t, \tau) & h_{12}(t, \tau) & h_{13}(t, \tau) & \cdots & h_{1 m}(t, \tau) \\ h_{21}(t, \tau) & h_{22}(t, \tau) & h_{23}(t, \tau) & \cdots & h_{2 m}(t, \tau) \\ \vdots & \vdots & \vdots & \ddots & \vdots \\ h_{n 1}(t, \tau) & h_{n 2}(t, \tau) & h_{n 3}(t, \tau) & \cdots & h_{n m}(t, \tau)\end{array}\right]$,

where $h_{n m}(t, \tau)$ is the time-variant channel impulse response between the $m^{\text {th }}$ transmit antenna and the $n^{\text {th }}$ receive antenna.

The channel matrix in (1) includes antenna-type effects, configuration, orientation, and frequency filtering. This knowledge could be instrumental in formulating a general multiple input multiple output relationship between the length- $m$ transmit signal vector $s(t)$ and the length- $n$ receive signal vector $y(t)$ given in (2).

$$
y(t)=\mathbf{H}(t, \tau) s(t-\tau) d \tau+\mathbf{n}(t)
$$

where $\mathbf{n}(t)$ gives the accompanying noise plus interference. For a time-invariant channel, the dependency of the channel matrix $t$ reduces to zero. Therefore, $\mathbf{H}(\tau=) \mathbf{H}(t, \tau)$. For instance, if the channel has a flat frequency, meaning there will be one single tap denoted by $\mathbf{H}$, then (2) reduces to (3).

$$
y(t)=\mathbf{H s}(t)+\mathbf{n}(t)
$$

2.2. Channel Model Requirements. In simple terms, mobile radio propagation models have diverging requirements to meet. On the one hand, the models should be very robust to provide adequate information about the propagation channels' properties. Here, the models' deficiencies should be stated such that there is no ambiguity in applying the models to the desired propagation environments. On the other hand, the models should be designed with simplicity for easy application, rapid implementation, and less computational complexities. Channel models developed in the past focused only on the aspects of key interests at the time. Such models might not give an accurate prediction of the radio channels if other environmental characteristics are considered. Given this, some existing models tend to present data on the received signal level and other key performance indicators (KPIs) [29, 30]. Typical examples of such models are the popular Okumura-Hata model [31], Lee's model [32, 33], and the COST 231 Walfisch-Ikegami model [34-36], which are most relevant to narrowband systems. Similarly, the COST 207-GSM [37], the International Telecommunication Union (ITU-R) [38-41], and the IEEE 802.11a/b models are most suitable for application in the $2 \mathrm{G}$ digital wideband systems [42]. For the $3 \mathrm{G}$ wireless systems, such as the UMTS [43-45], and IEEE 802.11n [46], and 4G systems such as LTE and LTE-A [27, 47-49], requiring smart antenna technologies, channel models that deal with directions of arrival and departure are inevitable for fast simulation, to reduce computational time, simplify system testing, and ease implementation. To this end, quality channel sounders are key requirements for developing more robust and efficient channel models for an environment of interest. 
2.3. Multilink Channel Sounding Techniques. In the past, the capabilities of most channel sounders are limited to singlelink measurements. As the number of links increases, there arises a need to design innovative solutions to characterize multilink propagation experimentally. In recent years, some techniques have been developed to measure multiple links. These include single-sounder sequential measurements, single-sounder multinode measurements, and multisounder measurements.

2.3.1. Single-Sounder Sequential Measurements. This technique focuses entirely on sequentially applying a single channel sounder. This means every connection is classically measured [50]. However, this method has some limitations because the propagation domain can change between runs [51]. The sounder calibration might not be stable, depending on each run's time difference relative to the clock's drift time. If the environment is maintained constant in the unlikely event, it is still challenging to achieve phase synchronism when considering multiuser measurements. However, if the environment is well controlled, this technique could be accurate and relatively cheap to implement [52].

2.3.2. Single-Sounder Multinode Measurements. In this technique, long RF cables can connect the equipment ports to distributed nodes [53]. It is worthy of note that the length of cables and the available signal-to-noise ratio (SNR) could pose severe limitations in using this technique. The measurement is only in the range of outdoor-to-indoor and indooronly conditions. Also, the cable movement may introduce phase jitter in mobile scenarios. Fiber optic cables are used to mitigate these limitations when a long distance is covered, but the cost of fiber and separately calibrating every cable is quite huge. However, this technique guarantees phase synchronization relative to the phase stability of the sounder. Sounders and their various architectures for MIMO channel measurements are discussed in [54].

2.3.3. Multisounder Measurements. The use of multiple MIMO channel sounders has proven to be the best option. Propagation measurements involving a single transmitter with multiple receivers are used in this technique. This technique is closely related to the real-world scenario. Still, the operation of two compatible sounders and maintaining multiple clocks' synchronization are essential requirements that come at a price. Further information on the multisounder measurement technique is provided in [55]. Last, MIMO channel sounders have been used to obtain measured data for model parameterization and validation $[11,56,57]$ and recently for modeling $5 \mathrm{G}$ and beyond $5 \mathrm{G}$ channels $[58,59]$.

2.4. MIMO Technology Classification. MIMO technology can be classified as point-to-point, multiuser, and massive MIMO. These are briefly described as follows.

2.4.1. Point-to-Point. This MIMO technology is the simplest of the three $[15,60]$. The BS is installed with an antenna array communicating with other terminals that are having an antenna array. The various terminals are multiplexed in an orthogonal manner, using time- and frequency-division mul- tiplexing techniques. Practically, point-to-point MIMO's usefulness can be limited even with massive arrays at the link terminals due to three key factors. First, the terminal equipment is quite complex, and it requires independent RF chains per antenna and the use of advanced digital signal processing techniques to separate the data streams. Second, the propagation environment should support minimum base stations and terminal antennas' independent streams. In a real-life scenario, this is difficult to achieve, mainly when compact arrays are used. Last, the spectral efficiency scales slowly with the minimum base station and terminal antennas near the cell edge, where ordinarily many of the terminals are located and where SNR is practically low due to severe path loss in the propagation paths $[6,61,62]$.

2.4.2. Multiuser MIMO. In multiuser MIMO (MU-MIMO), an antenna can provide network services to several UEs utilizing the same frequency resources $[6,63]$. MU-MIMO has some advantages over the point-to-point MIMO (P2PMIMO). MU-MIMO can use only single-antenna terminals. It is practically less affected by the propagation environment's dynamics, unlike point-to-point MIMO, which is easily affected by the conditions of line of sight (LoS). However, the practicality of MU-MIMO is limited by two factors $[64,65]$. The base station and the terminals must know the channel state information (CSI) on the downlink $[66,67]$. This can be very expensive, as enormous resources are required to transmit pilots in both directions efficiently. Second, advanced signal processing is highly needed for the BS and UEs for high spectral efficiency. Several wireless communication-related companies like Nokia Siemens, Eurecom, Intel, and Agilent Technologies, among others, treat multiuser MIMO as an adoptable candidate technology given by the Samurai project report [68]. The system is more feasible for mobile devices with low complexity and few reception antennas. To this end, an introduction to the multiuser MIMO downlink is given in [69]. Here, two algorithms comprising a signal processing with various types of transmitter beamforming and dirty paper coding [70] to solve the problem of signal interference from other users are well explained in simple terms. Multiuser MIMO description for the uplink and downlink scenarios is illustrated in Figure 2 [15].

2.4.3. Massive MIMO. A useful and scalable version of multiuser MIMO is referred to as massive MIMO (mMIMO) $[6,71]$. Massive MIMO is different from MU-MIMO in three instances. First, massive MIMO requires that only the BS needs to know about the channel state information. Second, the number of base stations is typically more significant than the number of terminal antennas. Last, there is no stringent requirement for complex signal processing techniques. This is because simple linear signal processing can be done on the uplink and downlink as indicated in several works $[5,7,72-77]$.

2.5. MIMO Channel Modeling. MIMO is presently a hot topic in modern wireless communication. Good models of the MIMO channel are ideal candidates required to compare 


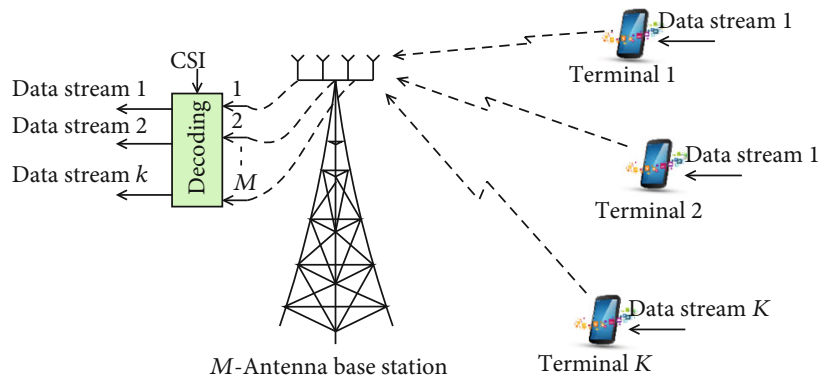

(a)

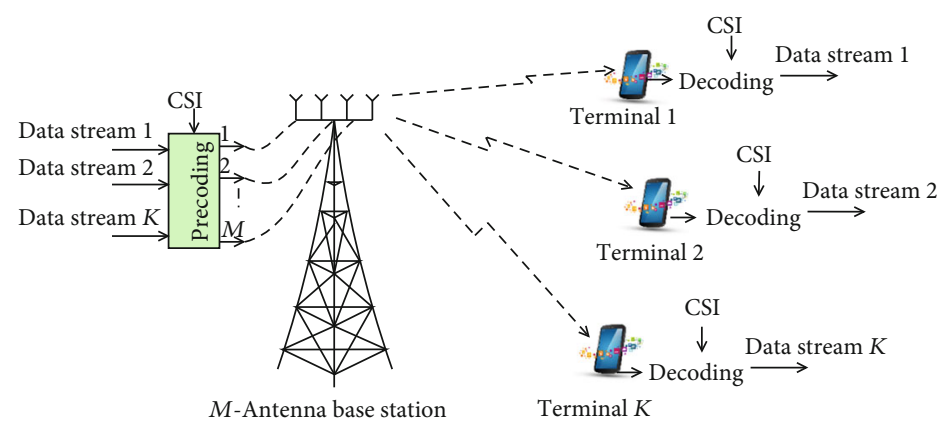

(b)

FIGURE 2: Illustration of multiuser MIMO technology: (a) uplink and (b) downlink.

various coding, modulation, and transmission schemes. These models often require that these models be detailed enough to accommodate the wireless channel's relevant characteristics. Furthermore, the models must be straightforward to ease implementation with speedy computational time. However, it is tough to compromise between simplicity and accuracy in wireless channel modeling [78]. Because of this, two approaches for modeling MIMO channels have been reported [28]. These include physical models and stochastic models. While physical models are aimed at modeling real-world physical parameters of the radio channels like the DoA and the DoD of all multipath components (MPCs), stochastic models present a description of the spatial channel properties by modeling the statistics at the antenna array elements.

A generic MIMO channel model can reproduce a couple of significant effects present in a MIMO channel. These are enumerated as follows. First, the direction of arrivals (DoAs) at the receiving array strongly depends on the direction of departures (DoDs) on the other link end's transmit weights. Second, the spatial properties of the channel differ for different delays of frequency-selective channels. Third, the number of scatterers in the model has to be finite. Fourth, the longterm spatial properties should reflect the clusterization of multipath components (MPCs) in delay and space. Fifth, the instantaneous MIMO transfer matrix can be rank deficient for the spatial covariance matrices at the link ends. Last, keyhole or pinhole channels must be adequately covered [79]. In a similar classification to the report in [80], MIMO channel models are broadly classified, as shown in Figure 3. In the next section, we shall present a detailed overview of the physical channel models.

\section{Physical Models}

Models of physical channels define the propagation environment based on double-directional electromagnetic wave propagation from the transmitting to the receiving arrays. The statistics of the parameters of the MPCs, which make up the total impulse response, can be easily modeled using the physical models. Interestingly, they model the parameters of the radio wave propagation like the direction of arrivals (DoAs) and direction of departures (DoDs) as complex amplitude values and delay of multipath components (MPCs). The models are independent of the antenna configurations, such as antennas, system bandwidth, mutual coupling, antenna pattern, polarization, and array geometry [17]. One of the simplest double-directional model's assumptions is that the DoAs and DoDs are separable [61]. Here, a joint power spectrum of the BS angular spectrum and the mobile spectrum are simply the two spectra' products. This allows directional channel models to be reused immediately. Second, geometrical models can assume single-scattering processes. In this case, the location of the scatterers determines the DoA and the DoD. These models are not suitable for microcellular scenarios. However, the models can be handy for modeling MIMO channels in macrocellular environments [81]. The physical MIMO channel models can be classified into deterministic physical models, geometrybased stochastic channel models, and nongeometrical stochastic channel models.

3.1. Deterministic Physical Models. Deterministic channel models tend to simulate the radio-propagation characteristics in the environment of interest. The simulation is done 




FIgure 3: Basic classification of MIMO channel models.

with a well-defined system setup to replicate the channel's transfer characteristics and the radio coverage specifically for a defined environment. These models find useful applications in the deployment or optimization of site-specific radio systems. They are also instrumental in channel evaluation during system design in crucial reference cases. Deterministic and ray models could be used to visualize the actual propagation mechanism, making them very useful channel modeling tools [28].

These models require the geometrical description of the environment under investigation. On the one hand, the geometric information is obtained from the given environment's database, using a digital file. Here, data for urban environments is saved in 3D vector format. In this case, the building walls and edges are depicted in $3 \mathrm{D}$ cartesian reference structures as collections of points or vectors. It was challenging to obtain such information in the past, but dense urban geographical structures [30] and building databases are fast becoming readily accessible at a reduced cost.

On the other hand, deterministic models could generate field strength outputs and a detailed explanation of the multidimensional channel transfer function over a network location, path, cell, or entire service region. These models provide quality information on time and space distribution, including delays in propagation, ray-paths, departure angles, and multipath arrival [82]. This is quite useful for planning $4 \mathrm{G}$ networks and the recently commercialized 5G wireless communication systems, adopting MIMO technology with multiple antennas and space-time coding schemes. Despite the numerous advantages of deterministic models, inaccuracies in database information, difficulties in modeling diffuse scattering, and high computational complexity are vital factors that limit the usefulness of the models. However, high expectations that the development of modern digital maps with more accurate geographic information systems will lead to significant improvements in GPS navigators in vehicular networks, and other mobile devices will further increase deterministic models' usefulness. In [83], the concept of double ring was used to explain M2M nonisotropic scattering environments, providing a new simulated deterministic channel model with a fair approximation of the desired statistical characteristics of the reference model. The concepts of ray tracing and stored measurements are briefly discussed as follows.

3.1.1. Ray-Tracing Algorithm. The propagation of electromagnetic waves through mobile communication channels is modeled by the ray-tracing algorithm [84]. The Tx and Rx locations are specified, followed by determining all possible routes or paths between the transceivers, using geometrical optics rules. Geometric ray tracing can be very challenging and time-consuming since this aspect of ray tracing requires technical skills and precision. Generally, a visibility tree can be adapted to capture the individual propagation paths. This tree has a well-layered structure, nodes, and branches [28]. Every tree node here portrays an entity of a building wall 


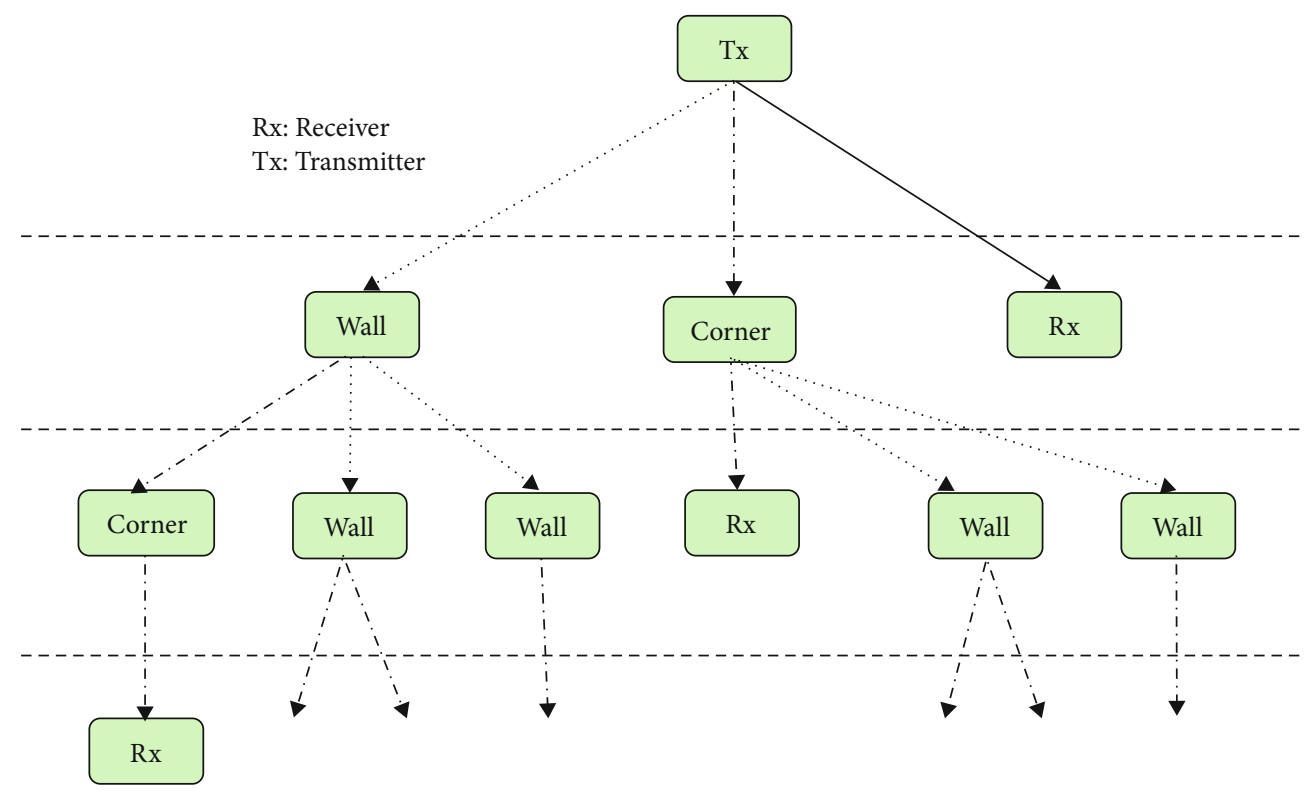

FIgURE 4: An illustration of a ray-tracing visibility tree.

and receives an antenna, and each branch of the tree represents an LoS link between two nodes, and the root nodes represent the transmit antenna as shown in Figure 4.

It is interesting to know that the tree's design follows a recursive approach like the typical tree, starting from the tree's root, from the transmitter to the receiver. In the first layer of the tree, the nodes correspond to all the transmitter has an LoS. A branch links the next two nodes in subsequent layers if there is a line of sight between the respective objects. Practically, a repeat of the process is carried out until reaching the highest layer in the prediction order. Furthermore, the branch corresponding to it is usually terminated with a leaf whenever the $\mathrm{Rx}$ is present in a layer. This means that the whole leaves on the tree equate to the number of tracks detected by the ray-tracing algorithm. Here, it should be noted that the design of a visibility tree can be challenging, especially for a full $3 \mathrm{D}$, if the prediction order is reasonably large. In particular, the concept of diffuse scattering was introduced in the ray-tracing technique to enhance its performance and the accuracy of prediction of the multidimensional propagation parameters and reduce the computational time. In [85], the concept of ray tracing was used to investigate and quantify the elevation characteristics such as height and distance dependency of key channel parameters, mean elevation angle, and elevation spread of a typical urban macrocellular environment. In [86], efficient multielement ray tracing was recorded, and the results were compared with site-specific measurements using calculated MIMO channel data. The ray-tracing algorithm has recently been used for links at $2 \mathrm{GHz}$ in built-up areas [87]. Unfortunately, ray tracing can be too computationally expensive and lacks flexibility since its effects are fundamentally related to the particular site in which they were extracted [88].

3.1.2. Stored Measurements. The concept of stored measurements was utilized in [89]. The sounder used for measurements, and the experimental set up recorded several SIMO snapshots as a Fast Doppler Block (FDB). Each snapshot comprises several complex uniform results along with the uniform linear array (ULA). A similar application of a sounder for measurements and storage is reported in [90]. The channel responses were estimated online and stored as a complex frequency response for subsequent offline postprocessing on the sounders' hard disk. This methodology is greatly affected by many memory requirements, coupled with the understanding that the channel responses obtained are site-specific [88].

3.2. Stochastic Models. These models use statistical means to estimate the spatial features of MIMO-based radio channels. These spatial features or properties are often referred to as the channel's correlation properties [91, 92]. A plane wave that impinges upon an antenna array from a specific direction gives the steering vector. Notably, the correlation properties are dependent on the channels of spatial characteristics and the configurations of the antenna array. Stochastic models tend to model the MIMO channel as experienced by communication systems applying specific antenna arrays. Details on the second-order statistics obtained from the full MIMO correlation matrix can be quite large. The elements can be challenging to interpret directly concerning physical propagation.

3.2.1. Geometry-Based Stochastic Models (GBSMs). In the GBSM model, the scatterers are seen to be stochastically distributed across the two ends of the link. In this case, the fundamental laws governing diffraction, reflection, and dispersion of electromagnetic waves are utilized to derive the channel model from the scatterers' positions. It should be noted that the shape of the scattering area gives information about the tested scenario. Through a careful distribution of scatterers around the UE, macrocells can be appropriately simulated. On the other hand, the microcells include ellipses, which have focal points at the NodeB and the UE [88]. 
Furthermore, some parameters, like the number of scatterers in the scattering region and creating them into clusters, are crucial to achieve a reliable simulation of the particular propagation scenario. In a situation where the scatterers are distributed evenly in and around a circle positioned on the UE, analogous to Clark's Doppler spectrum, it becomes easy to achieve uniform distribution of the DoA in the azimuth, and typical model that accounts for double bounces has been presented in the following reports [93-95].

3.3. Geometry-Based Statistical Propagation Models. The geometry-based statistical propagation models are briefly discussed under the one-ring model, a combined elliptical model, and the one-disk model.

3.3.1. One-Ring Model. The one-ring model appears to be the most popular geometry-based stochastic channel models proposed in line with MIMO systems in $[96,97]$. The model follows an approach that accounts for local dispersion with the subscriber unit from obstacles nearby. This is achieved through the appropriate placement of scatterers on a ring. This model finds useful applications in MIMO systems, as presented in [98]. The one-ring model may not necessarily follow the popular Kronecker model [98]. For instance, when the antenna separation distances are chosen so that the transceivers' correlations are forced to a zero value, the crosscorrelation in the $2 \times 2$ broadside configuration equals -0.24 and 1. This is quite far from the Kronecker assumption. The set of equations detailing the different correlation coefficients for the model are also available in [98].

3.3.2. Combined Elliptical-Ring Model. A combination of isodelay ellipses was explored for the defined tap-delay profile and angular spreads, leading to the creation of the combined elliptical-ring model in [99]. Typically, this model is characterized by a vital parameter called local scattering ratio (LSR). This ratio and the angular spreading properties are directly related. For example, an LSR indicates an omnidirectional spread of scatterers on the local ring, whereas a low LSR represents scatterers' distribution in a given direction [100, 101].

3.3.3. One-Disk Model. The one-disk model is very similar to the geometry-based model for the LoS multipath radio channel reported in [102]. The model consists of a reasonably large single disk located between a transmitter and a receiver and loaded with several evenly spaced scatterers. This model's correlation properties are highly dependent on the path loss exponent, and this property clearly distinguishes the one-disk model from the other geometry-based statistical propagation models. However, the antenna correlation can be relatively low, and the magnitude of the cross-correlations for the one-disk model can be relatively high.

3.4. Nongeometrical Stochastic Physical Models. These models only define paths between transmitter and receiver by statistical means without considering physical environments' geometry. Examples of such models include the SalehValenzuela model, the extended Saleh-Valenzuela model, which uses MPC clusters, and the Thomas Zwick model, which manages MPCs individually.
3.4.1. Saleh-Valenzuela Model. Following the pioneering work reported in [103], Saleh and Valenzuela [104] present a model, which gives information about the arrival time of the MPCs in the delay domain for a wireless time-invariant channel. Leveraging on the pioneering work in [103], SalehValenzuela considered the channel response and broke it into clusters, each comprises several multipath components. Typically measured parameters for the Saleh-Valenzuela model in an indoor, fixed point-to-point office environment are documented [104].

Key contributions of the Saleh-Valenzuela model are worth mentioning. First, the model is among the earliest models to consider the concept of clustering as related to multipath. Second, the clusters' arrival times and the multipath components within each cluster were modeled as two independent Poisson processes, each with a different rate. Third, the model showed the splitting of the received probability density profile (PDP) into several PDP components and an overall PDP. Also, the model revealed that both PDPs could be modeled as exponentially decaying profiles. The model has gained widespread popularity due to its accuracy and simplicity. Key elements of the model serve as foundation blocks for the development of most standard channel models, including the popular 3GPP spatial channel (SCM), COST 259, COST 273, and the IEEE 802.15 ultrawide band (UWB) models [105].

3.4.2. Extended Saleh-Valenzuela Model. While working at Bells Laboratories, Saleh and Valenzuela [104] performed extensive measurement campaign from many fixed indoor point-to-point locations. They provided a heuristic estimation of the parameters using curve-fitting techniques. The results of their investigation revealed that MPC clusters could be modeled in the delay domain through a double exponential-decay process. This modeling approach is often described as the Saleh-Valenzuela (SV) model. However, the sounder used by Saleh and Valenzuela for the radio propagation measurements utilized antennas that were omnidirectional in the azimuth plane.

Consequently, the model omits any AoA and AoD characterization. Several studies have reported extensions to the model to widen its application to include the azimuth and elevation characteristics. For example, in [106], PDPs were measured in the azimuth domain, extending the SalehValenzuela model to include the azimuth AoA. Similar to the SV model, the resulting model is only applicable to the time-invariant channel. Furthermore, the resulting model from work in [107] clustered in the delay domain, and multipath components were seen clustered in the azimuth domain. It is worth mentioning that the azimuth clustering followed a Laplacian distribution. In the extended SV model, the channel impulse response in the azimuth-elevation space reflects a summation of all multipath components' contributions in all clusters (4) given by [108].

$$
h(\tau, \varphi)=\sum_{c=0}^{\infty} \sum_{\ell=0}^{\infty} a_{c}, \ell e^{j \varphi_{c}, \ell} \delta\left(\tau-\tau_{c}-\tau_{c, \ell}\right) \delta\left(\varphi-\varphi_{c}-\varphi_{c, \ell}\right)
$$


where the variables $\varphi_{c}$ and $\varphi^{\prime}$ describe the azimuth AoA of the clusters and the MPCs, respectively. Notably, $\varphi_{c}$ stands for the mean value of the AoA of all multipath components within that cluster. Also, $\varphi_{c}$ and $\varphi^{\prime}$ are random variables. If the AoA of the first cluster is given, the conditional distribution is modeled assuming a uniform distribution within $U(0,2 \pi)$. The multipath component distribution is modeled as a zero-mean Laplacian in (5), also given by [108].

$$
p\left(\varphi_{c, \ell}\right)=\frac{1}{\sqrt{2 \sigma \varphi_{c, \ell}}} \exp \left(-\frac{\sqrt{2}}{\sigma \varphi_{c, \ell}}\left|\varphi_{c, \ell}\right|\right),
$$

where $\sigma_{\varphi_{c, \text { e }}}^{2}$ is the azimuth spread at the receiver, and it is an empirical quantity.

3.4.3. Zwick Model. The Zwick model is typically a stochastic model suitable for indoor channel propagation [109]. The model uses geometry based on a death-birth technique for modeling the appearance and disappearance of the motion of multipath components concerning time. In this case, it becomes straightforward to monitor spatial and temporal correlations. The model uses a ray-tracing tool, which can be very expensive, to allow automated extraction of parameters for new environments and different frequencies without the need for actual field measurements. However, the model enables Monte Carlo to simulate wireless communication systems, especially smart adaptive antennas, coding, and modulation. Here, path properties can be observed following the BS and MS's motion, which results in a practical Doppler activity of the signal. This model also enables smooth transitions and achieves results with lesser computational time, between LoS and the obstructed line of sight (oLoS) configurations, by modeling the LoS portion separately from other multipath components. The model could be used to carry out system simulations for the bit error rates (BERs) of radio links and intelligent antenna configurations such as SDMA, MIMO, and spatial filtering for interference reduction (SFIR) [110].

In the preceding section, we have presented an elaborate description of the physical channel models. Next, we shall explore the analytical channel models.

\section{Analytical Models}

Analytical models tend to model the radio channel's impulse response between the transmitters and receivers on an individual basis, without providing adequate information about the wave propagation. This contrasts with the physical models, which tend to show the statistics of the parameters of MPCs that make up the total impulse response. In this case, the individual channel responses could be taken as the MIMO channel matrix. The analytical channel models find a wide range of useful applications in simulation environments because they are easy to generate and can be very useful in representing various channel responses. Here, it is worth noting that there is no direct correspondence between the physical environment where the MIMO system operates and the channel matrix. Generally, these models are further classified into the correlation-based and the propagationmotivated analytical channel models. Also, the analytical models based on space-time coding are briefly highlighted.

\subsection{Correlation-Based Analytical Models}

4.1.1. The i.i.d Model. The independent and identically distributed (i.i.d) model is often described as the simplest MIMO model. In some literature, it is called the canonical model. In the model, $R_{\mathbf{H}}=p^{2} I$, where $p^{2}$ is referred to as the channel power and $\mathbf{H}$ is used to denote the MIMO channel matrix. The matrix comprises elements, which are uncorrelated and statistically independent and of equal variance $p^{2}$ [111]. This phenomenon is often observed in spatially white MIMO channels over a robust scatterer environment defined by uniformly distributed independent MPCs. This model consists of the channel power used in information theory for analyzing MIMO systems. The i.i.d model describes the application of multiple elements for a single user over the additive Gaussian channel. Due to its characteristic behavior, the model is statistically independent and unable to achieve correlation. However, most systems exist in environments with limited scatterers and small antenna spacing between the Tx and Rx. The implication is that there exists a strong correlation between the channel matrix elements and mutual coupling between the specified elements of the antenna array on the link ends. Hence, it can be suggested that the i.i.d model and its capacity gains are most suitable for theoretical analysis.

4.1.2. Kronecker Model. The Kronecker model assumes that the scatterers at both link ends can be treated independently. Given this separability assumption, the model approximates the full correlation as the Kronecker product of one-sided correlation matrices. Consequently, the number of parameters needed is reduced to provide useful information about the channel and introduces some structure to the model. The correlation matrices are a clear indication of the scattering seen at either link end. Despite the numerous reports suggesting that the Kronecker model is inaccurate for most practical scenarios, it is, to no small extent, the most widely used MIMO channel model because of its ease of application and analytical tractability [112]. The Kronecker model could also be used to model Tx and Rx's correlation properties separately, but it often ignores the dependence of the DoAs on the DoDs [61]. The Kronecker model only presents the full correlation matrix as the Kronecker product of the two marginal correlation properties at both link ends. Any DoD will result in the same DoA. This is usually not valid for most practical MIMO channels since the model is seen to predict the capacity of the channel inconsistently [96, 98, 113-115]. The wideband equivalent synthesis equation of the model is given in (6) by [116].

$$
\mathbf{H}_{\mathrm{Kron}}=\frac{1}{\sqrt{\operatorname{tr}\left\{\mathbf{R}_{\mathrm{Rx}}\right\}}} \mathbf{R}_{\mathrm{Rx}}^{1 / 2} \mathbf{G}\left(\mathbf{R}_{\mathrm{Tx}}^{1 / 2}\right)^{T}
$$

As given in (6), $\mathbf{R}_{\mathrm{Tx}}=E\left\{\mathbf{H}^{T} \mathbf{H}^{?}\right\}$, and $\mathbf{R}_{\mathrm{Rx}}=E\left\{\mathbf{H} \mathbf{H}^{H}\right\}$, represent the $\mathrm{Tx}$ and $\mathrm{Rx}$ correlation matrices, respectively. 
Also, $\mathbf{G}$ is an i.i.d unity-variance random fading matrix with complex Gaussian variables.

4.1.3. Weichselberger Model. To improve the Kronecker model, a more realistic channel model was provided by Weichselberger [117]. Here, the transmitter and the receiver's joint correlation are modeled while preserving the dependencies between the DoAs and DoDs. The Weichselberger model comprises three components. First is the correlation matrix of the spatial eigenbasis of the transmitter and the receiver. Second, the receiver and transmitter eigenmode pair, which spans the SISO channel. Next, the powercoupling matrix of each element is formed by its average energy.

It should be noted that these eigenmode SISO channels are mutually completely not correlated [61]. When the coupling matrix is the same as the transmitter's outer product and receiver eigenvalues, the Weichselberger model approximates the Kronecker model. The implication is that the coupling matrix would reduce to rank one. The eigenvalue decomposition of the receiver and transmitter correlation matrices are given in (7), and the Weichselberger synthesis is given in (8).

$$
\begin{aligned}
\mathbf{R}_{\mathrm{Rx}} & =\mathbf{U}_{\mathrm{Rx}} \Lambda_{\mathrm{Rx}} \mathbf{U}_{\mathrm{Rx}}^{H}, \mathbf{R}_{\mathrm{Tx}}=\mathbf{U}_{\mathrm{Tx}} \Lambda_{\mathrm{Tx}} \mathbf{U}_{\mathrm{Tx}}^{H}, \\
\mathbf{H}_{\text {weichsel }} & =\mathbf{U}_{\mathrm{Rx}}\left(\tilde{\Omega}_{\text {weichsel }} \Theta \mathbf{G}\right) \mathbf{U}_{\mathrm{Tx}}^{T},
\end{aligned}
$$

where $\mathbf{G}$ gives the i.i.d, a complex Gaussian random fading matrix, and $\tilde{\Omega}_{\text {weichsel }}$ describes the element-wise square root of the power coupling matrix $\Omega_{\text {weichsel }}$. Further explanation of the variables in the model is given in [116].

4.1.4. Virtual Channel Representation (VCR). The VCR tends to model the channel in the beam space, using predefined steering vectors given in [118]. In mathematical form, the VCR model is given in (9) by [116].

$$
\mathbf{H}_{\text {virtual }}=\Lambda_{\mathrm{Rx}}\left(\tilde{\Omega}_{\text {virual }} \Theta \mathrm{G}\right) \Lambda_{\mathrm{Tx}}^{T}
$$

with $\boldsymbol{\Lambda}_{\mathrm{Rx}}$ and $\boldsymbol{\Lambda}_{\mathrm{Tx}}$ and $\tilde{\Omega}_{\text {virtual }}$ denoted as the element-wise square root of the power coupling matrix $\Omega_{\text {virtual }}$ in the virtual domain. In this case, the number of virtual angles will help determine the VCR model's accuracy and angular resolution. It is interesting to note that the number of virtual angles cannot be assumed at random but obtained from the antenna array configurations. In practice, the virtual channel representation is seen to restrict the eigenbases shown to be of one-sided correlation matrices to predefined digital Fourier transform (DFT) matrices elaborated in [117]. This concept makes the DFT matrices perform an asymptotic optimal eigenfunction for the channel matrix [118]. Also, samples of the uncorrelated spectral features are formed by the elements of the fading matrix $\tilde{\Omega}_{\text {virtual }} \Theta \mathrm{G}$. One key component of the VCR is the ease with which it can be interpreted. The antenna configuration determines its angular resolution accuracy. The accuracy increases relative to smaller angular bins as the number of antennas grows.
4.1.5. Diagonal-Decorrelation Model. The diagonaldecorrelation model tends to equate all cross-correlations to zero independently with antenna correlation values. Here, the channel matrix $R$ is assumed to remain semipositive definite. This model has been reported in [119-121] with details limited to physical justification.

4.1.6. Rough Surface Model. The rough surface model was proposed in [122] to model multiuser MIMO channels. This model takes its root from Kirchhoffs theory [123] on the derivation of cross-user and intrauser correlations for two near located users. The model enables the study of the antenna pair correlation across users and within a single user. Here, the physical environment's essential features, such as the scattering surface's roughness and geometry, are characterized. The scatterer is modeled such that it resembles a rough surface, having a height defined as a Gaussian process. Points on the uneven surface scatter the incident wave in different directions with a given probability. Correspondingly, this leads to multiple user signal correlation.

This model could apply to model users located within a valley and scatterers located in streets. The model is useful in estimating the spatial correlation relative to the scattering surface profile and the channel gain's theoretical limit, and it could be extended to multiuser channel models. The model could also be advantageous in locations where the scatterer's random rough surface modeling is achievable.

For the model, theoretical derivations for the inter- and intrauser correlations were estimated using numerical methods. Here, LoS signals are seen to enhance the correlation with a flatter trajectory in space for the inter- and intrauser cases. This feature could be explored to distinguish LoS signals from their NLoS counterparts clearly. A comparative analysis of the Kronecker model, VCR model, Weichselberger model, and the rough surface model is reported in Table 1.

4.1.7. The Structured Model. The structured model is an extended version of the Weichselberger model. It adopts the tensor calculus concept in describing the scatterers' coupling across the receiver's delay space and the transmitter. Three correlation matrices that describe the correlation around the receiver, transmitter, and delay space have been highlighted in this model, using tensor calculus. The structured model approximates the correlation across receivetransmit-delay space. There is no independence assumption between the scatterers at the receiver, transmitter, or different delays as in the Kronecker model. This is why this model better approximates the actual structure of the wideband MIMO channel. This model also requires fewer parameters than the Kronecker model for the synthesis of an ensemble of $H$ -tensors. In most practical scenarios, reports show that this model consistently outperforms the well-known Kronecker model, using several metrics [124].

4.2. Propagation-Motivated Analytical Models. Models such as the finite scatterer and the maximum entropy tend to describe the MIMO channel matrix, employing appropriate 
TABle 1: A comparative description of some MIMO channel models.

\begin{tabular}{|c|c|c|c|}
\hline Models & Suitable environments & Benefits & Limitations \\
\hline Kronecker & Local scatters only & Mathematically simple & A special case of VCR \\
\hline $\begin{array}{l}\text { Virtual } \\
\text { channel } \\
\text { representation }\end{array}$ & $\begin{array}{l}\text { Scatters distribute mainly } \\
\text { according to predefined } \\
\text { angular directions }\end{array}$ & $\begin{array}{l}\text { Capturing the impact of scattering parameters on } \\
\text { channel diversity and capacity }\end{array}$ & $\begin{array}{l}\text { Requiring specific scatter } \\
\text { distribution or large array }\end{array}$ \\
\hline Weichselberger & Versatile environments & The limitations on VCR being lifted & $\begin{array}{c}\text { They require clustered antennas at } \\
\text { the Tx and } \mathrm{Rx} \text {. }\end{array}$ \\
\hline $\begin{array}{l}\text { Rough surface } \\
\text { model [122] }\end{array}$ & Large plane scatters & $\begin{array}{l}\text { Theoretically computing the spatial correlation relative } \\
\text { to the scattering surface profile and the channel gain } \\
\text { and extending to multiuser channel models }\end{array}$ & $\begin{array}{l}\text { Restricted to specific environments } \\
\text { and requiring some parameters of } \\
\text { the scattering surface }\end{array}$ \\
\hline
\end{tabular}

propagation parameters. A brief description of these models is as follows.

4.2.1. Finite Scatterer Model. This is a well-known propagation-motivated analytical model. This model uses a limited number of multipath components for the modeling of the channel. In contrast with the GSCM models that accommodate only single and double-bounce scattering, this model gives room for a single-bounce and multiple or several bounce scattering. It should be added that this model allows for split components, having a single direction of departure and then splitting into two or several paths with different directions of arrivals afterward. Here, the split component is seen as multiple components having the same DoA or DoD. The MIMO channel matrix for the narrowband case of this model is presented in [28]. It is worthy of note that this model is fully compatible with the 3GPP spatial channel model [125]. The reader can find additional information on this model in related reports [126-128].

4.2.2. Maximum Entropy Model. Another well-known propagation-motivated analytical model is the maximum entropy model proposed in [111]. This model gives the distribution of the MIMO channel regarding useful information available on the channel. Usually, this preliminary information may consist of the propagation domain features and system parameters such as bandwidths and arrivals' directions, among others. For consistent results from the model, it is reported in [111] that the choice of the model influences the type of application. This means that different channels of the model may be evaluated for capacity estimation than for the simulation of BER. In conclusion, the maximum entropy model is straightforward to apply, mainly when translating physical information into the channel's distribution model that requires consistency.

4.3. Analytical Models Based on Space-Time Coding. These are a class of propagation models that have been developed for theoretical analysis of communication systems based on space-time coding reported in several works of literature [129-134]. These models characterize the communication channel in terms of degrees of freedom, allowing precise analysis of the channel capacity following the reports [135137]. The interest in this class of analytical models is increas- ing due to the development of $6 \mathrm{G}$ wireless networks to achieve the much anticipated ubiquitous connectivity [138].

In particular, the limit on the available number of spatial degrees of freedom is derived for multiple-antenna systems that suffer the limitations posed by the area and geometry of large antenna arrays [135]. The work is aimed at defining the spatial signal space to describe channel propagation measurements better to characterize the physical environment under investigation. The optimal number of antennas required for a given wireless device in a well-defined application environment is determined. A typical illustration of the clustering of transmitting and receiving signals showing the clustering intervals in the multiple-antenna channels is described by [135] in Figure 5.

Similarly, in [136], the authors considered the number of degrees of freedom of the field in MIMO channels. The work is aimed at examining the relationship between the number of degrees of freedom (NDF) of the field and the effective number of degrees of freedom (ENDF) of a MIMO channel. The work also examines the channel capacity of a pure spatial communication system corrupted by AWGN. The spatial band limitation properties of the field were employed in evaluating the maximum ENDF. Results indicate a strong relationship between the NDF of the field and the MIMO system's best performance. More specifically, the field's NDF poses a significant limitation to the largest number of SISO subchannels. The results find useful applications in microwave tomography and inverse source detection.

In [137], a numerical evaluation of the electromagnetic (EM) degrees of freedom (DoF) of a noise-limited system in $2 \mathrm{D}$ with multiple random scattering is presented. The received EM fields were obtained using the fast multipole method (FMM), and the Monte Carlo simulation was adopted to determine the ensemble average of the DoF number. The results show that the transmit and receive volume sizes and the scattering region determine the average EM DoF number. Specifically, the transmit and receive volumes tend to increase the average number of DoF, though the observed increase is a nonlinear process.

\section{Standardized Models}

In the preceding section, we provide an exhaustive survey of the analytical channel models. Next, we shall examine the standardized channel models in this section. 


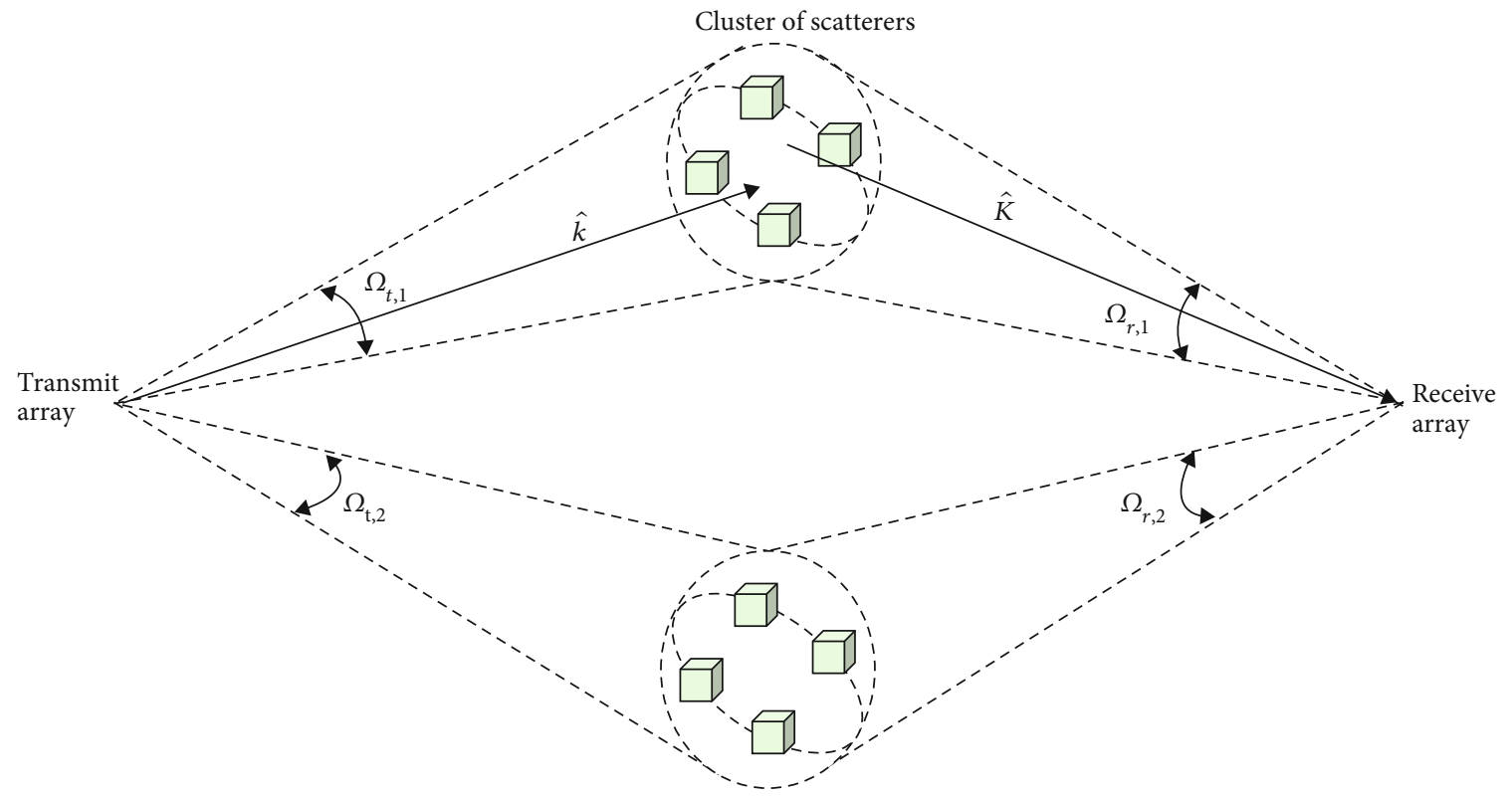

Figure 5: A typical illustration of the clustering of transmitting and receiving signals showing the clustering intervals in the multiple-antenna channels.

The standard models present a unified framework for the development of new radio systems. Different techniques, such as signal processing and multiple access, are incorporated into such models to improve performance and enhance system capacity [61]. An example of the standardized model is the wideband COST 207 power delay profile model. This model is used for GSM development in terms of choosing appropriate modulation and access techniques. Other directional standardized MIMO models include the COST action projects, 3GPP SCM, WINNER channel, IEEE 802.11n [46], IEEE 802.16ac, and the Stanford University Interim (SUI) models. These are briefly described as follows.

5.1. COST Action Projects. The European COST action initiative has developed several models, including the wireless channel's directional features that apply to MIMO and smart antenna simulations. Starting with the COST 207, following rapid improvements leading to the COST 2100 model's emergence, the COST action project has triggered wireless communication systems' growth and development. These models are briefly presented in Table 2.

5.2. 3GPP Spatial Channel Models. In this section, we present the 3GPP/3GPP2 spatial channel model and the extended 3GPP spatial channel model as follows;

5.2.1. 3GPP/3GPP2 Spatial Channel Model. The 3GPP spatial channel (SCM) model was developed to aid the simulations of macrocells and microcell deployment in $3 \mathrm{G}$ networks with a bandwidth of $5 \mathrm{MHz}[148,149]$. In this model, the azimuth spread, DoAs, and DoDs were assigned constant values. Unlike the COST, the model approach is quite unconventional, which allows for continuous large-scale movement of user devices. The model takes into consideration the different aspects of the user device's motion within the coverage area. Tap-delay line parameters are provided for the implementation of this model. Here, each tap-delay line has multiple subpaths sharing different DoAs and DoDs with the same delay. In the model, interference is categorized into strong and weak interference. Weak interferers are modeled as spatially white, and strong interferers are characterized as spatially correlated. Considering multiple mobile device connection to a single BS and a single mobile device connection to multiple base stations, the values of the shadowing correlation are given as 0 and 0.5 , respectively.

5.2.2. Extended 3GPP Spatial Channel Model. An interim channel model for use in emerging technologies other than $3 \mathrm{G}$ systems was reported [150]. It is worthy of note that this model is backward compatible with the 3GPP SCM. Meanwhile, the original SCM is a ray-based model following the stochastic modeling of scatterers. The model has been implemented in MATLAB, following the report in [151].

The model classified the urban micro, urban macro, and suburban macro environments. Here, urban micro has different characteristics for NLoS and LoS propagation. There are six fixed paths for each scenario depicting the Dirac delay function consisting of twenty subpaths spatially separated. This model follows directly from the sum-of-sinusoids method. The SCM is only defined for CDMA at $5 \mathrm{MHz}$ bandwidth, and the model is limited in terms of systemlevel simulations for time-varying channels. The interim beyond the $3 \mathrm{G}$ channel model was proposed partly due to its simplicity and backward compatibility to alleviate these shortcomings. The extension to the SCM focuses on bandwidth, frequency range, and other extensions such as LoS scenarios, time evolution, and tapped-delay line modeling characteristics [152-154].

Some features such as the intrapath delay spread (DS) inherently missing from the SCM model are added on 
TABLE 2: The early COST action projects.

\begin{tabular}{|c|c|c|}
\hline S/N. & COST model & Model description \\
\hline 1 & COST 207 channel model & $\begin{array}{l}\text { COST } 207 \text { is the earliest of the COST action projects [37]. This model proceeds from the Digital Land } \\
\text { Mobile Radio communications project from September } 1984 \text { to September 1988. The project is aimed at } \\
\text { supporting the Confederation of European Posts and Telecommunications (CEPT) GSM to coordinate } \\
\text { research in propagation, modulation, and coding. } \\
\text { The propagation activity themes focused on field strength prediction techniques, radio channel } \\
\text { measurements, characterization, radio channel simulation, antenna, and manufactured noise. During the } \\
\text { project, existing path loss prediction techniques were reviewed, and information from the terrain and } \\
\text { land cover was carefully obtained to form a topographic database. } \\
\text { The study also investigated the design of diversity antennas for base stations, vehicle-mounted, and } \\
\text { handheld applications. The impact of the vehicle structure on the radiation characteristics of the antennas } \\
\text { was analyzed. The study also specified the PDP for the environments classified as typical urban (TU), bad } \\
\text { urban (BU), reduced TU, reduced BU, rural area (RA), and hilly terrain (HT). It is worthy of note that the } \\
\text { COST } 207 \text { project prepared a foundational framework for developing the COST } 231 \text { model. }\end{array}$ \\
\hline 2 & COST 231 Hata model & $\begin{array}{l}\text { The COST } 231 \text { Hata model is based on a proposal for extending the popular Okumura-Hata model } \\
\text { to the } 1500-2000 \mathrm{MHz} \text { band, specifically for propagation environments where the Okumura-Hata } \\
\text { model underestimates the path loss. The specifications of the model can be found in several reports } \\
\qquad[35,139-144]\end{array}$ \\
\hline 3 & $\begin{array}{l}\text { COST } 231 \text { Walfisch- } \\
\text { Ikegami model }\end{array}$ & $\begin{array}{l}\text { Interestingly, the COST } 231 \text { Walfisch-Ikegami model classified the propagation link by distinguishing } \\
\text { between LoS and NLoS suited for microcellular cells. The operating frequency span } 800 \mathrm{MHz} \text { to } \\
2000 \mathrm{MHz} \text { and a receiver-transmitter separation distance of } 0.02-5 \mathrm{~km} \text {. Details of the LoS and NLoS } \\
\text { scenarios and other useful parameters of the model are given in [34, 145-147]. }\end{array}$ \\
\hline
\end{tabular}

bandwidth. This addition is done so that the model's characteristics are unaltered compared with the original SCM at $5 \mathrm{MHz}$ resolution bandwidth. Saleh and Valenzuela earlier proposed this approach of intrapath delay spread, and it has been used for an outdoor environment in the COST 259 model [155, 156]. Further to this, midpaths moved to different delays with the original path were developed by splitting the twenty subpaths into subsets. The midpath specifications, including the delay profile, power, and midpath number, are presented in [150]. The power of the midpaths was estimated by computing the decreasing power relative to the delay with limits taken, more significant than the least number of subpaths. Details on the method used to derive the delays are reported in [150]. Other extensions to the SCM are given in $[148,155,157]$.

\subsection{The COST 259 Directional Channel Model. The COST} 259 is the first model in the COST action projects that considered complex relationships between the propagation distance, angular spread, delay dispersion, and other channel parameters [158]. The channel delay and angular dispersion are seen at the base station, and mobile station under different radio propagation conditions are modeled using this model $[159,160]$.

The objectives of the COST 259 were focused on agreement with measurements, consistency, simplicity, and completeness. First, the model's fundamental goal is to reproduce many of the characteristics derived from measured data. Second, the model should be backward compatible. Since the model is built on existing COST projects like the early COST 207 model, whose constructs are used for remarkable realizations, the model is expected to be consistent with its older versions. Third, the model is developed such that it is mathematically tractable and of low computa- tional complexity. Macro, micro, and picocells are modeled, and frequencies ranging from $800 \mathrm{MHz}$ to $5 \mathrm{GHz}$, covering both outdoor and indoor scenarios, are included in the model [158].

This physical model provides a general overview of the radio propagation site. The coverage includes macrocellular, microcellular, and picocellular scenarios such as the urban, bad urban, open-square, outdoor domain, and indoor office. The modeling approaches for macro-, micro-, and picocell cells vary widely in this model $[158,161]$. Each radio environment is defined in the macro approach by external parameters such as base station positioning, average base station, and mobile station height, radiofrequency, and a set of functions of probability density and statistical moments defining the given propagation site. A Poisson distribution, for example, characterizes the number of scatterers. The model could be treated as both partly stochastic and geometric to evaluate the global parameters. Besides, the MS can be positioned within the BS coverage at random. This positioning makes it easy to calculate the delay and mean angles of the various clusters, which constitute the doubledirectional impulse response. The angular distribution, the delay's distribution, and the shadowing are stochastically determined. These are seen as lognormally distributed, correlated random variables.

For the global parameters' realizations, local parameters are randomly generated in each radio environment comprising multiple propagation environments. Local parameters are presumed to be approximately constant and define the instantaneous channel behavior. The COST 259 model can manage MS's continuous movement through multiple propagation environments and various radio environments.

Modeling constructs: basically, four methods are suggested to simulate the channel impulse response (CIR) 


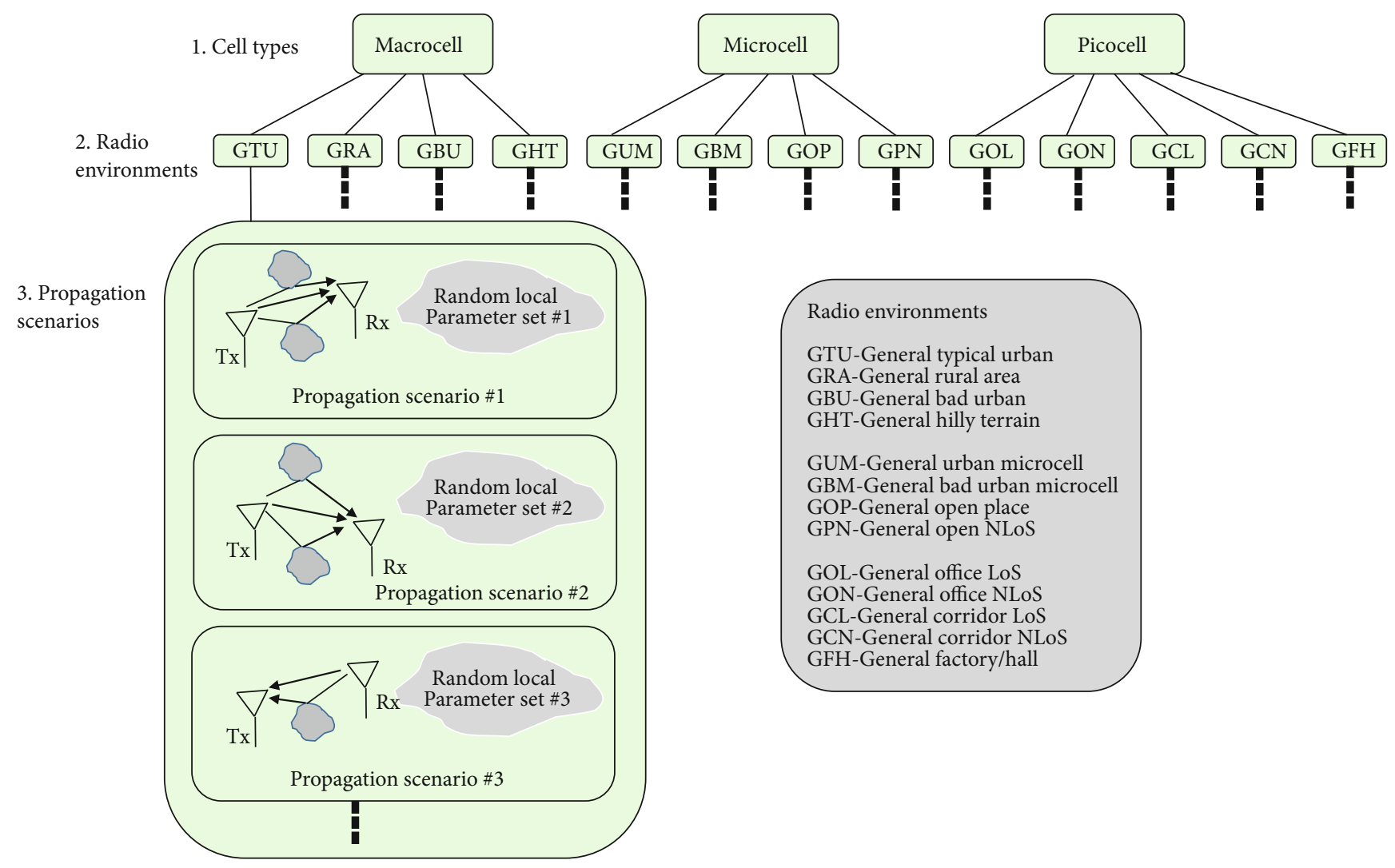

FIGURE 6: A layered structure depicting the COST 259 directional channel model.

functions in the COST 259 model. These are stored ray CIRs, optical approaches, stochastic models of radio channels, and stochastic parametric models, as given in [159].

Stored CIRs are defined to be the most accurate and realistic CIRs. However, it is challenging to incorporate all environmental effects on a particular set of stored CIRs. If a large group of CIRs is used, there is a considerable price for storage. Besides, it is very difficult to share stored measurement results with the public. Second, the ray-tracing outcomes are much easier to derive compared with observed CIRs. However, an extensive database is often required for effective ray tracing, and a precise definition of the propagation effects is of paramount importance.

Appropriate modeling of stochastic radio channels helps to characterize the CIR as the stochastic process's product properly. Also, the Widesense Stationarity Uncorrelated Scattering (WSSUS) model utilizes power spectral densities or correlation functions to classify propagation channels $[162,163]$. This modeling approach was adopted for the COST 207 model, and the parametric method is used for most aspects of the COST 259 model. The WSSUS property for parametric stochastic models, however, is not too necessary. Here, incident waves at the receiver are usually defined by some parameters like the delay and DoA, not their correlation functions.

5.3.1. Propagation Scenarios. Fundamentally, radio wave propagation relies on the topography and electromagnetic characterization of the environment under investigation. A well-defined three-layered structure has been proposed for the model [159]. For example, the popular COST 259 directional channel's layered structure is illustrated in Figure 6. The layered structure eases categorizing channel models according to their applications in different types of systems.

The top layer shows different cell types, such as macro, micro, and pico. For all cell types, radio environments are attached. All radio environments' names start with the word generalized, indicating an extension of the COST 207, as shown in the middle layer. As shown in the third layer, modeling constructs' layered structure comprises the various propagation scenarios (PSs), depicting random realization of multipath conditions. There are three scenarios, which are denoted as 1, 2, and 3 propagation scenarios.

Interestingly, the PSs are not strictly classes, but they represent small-scale channel states, each showing a constant power delay direction profile (PDDP) and scattering function. In most practical scenarios, large-scale parameters within a PS are seen to be constant. This implies that a PS can only represent the propagation conditions within a specified physical area.

Parameter types: here, we look at the parameters that define radio environments. These parameters are external, global, and local parameters. The external parameters include the average heights of the BS and MS antennas, the frequency band, the average distance of the transceivers, average height, and separation of the structures being considered. Further to this, specifying the LoS and NLoS condition requirements is a prerequisite in many REs. However, in 


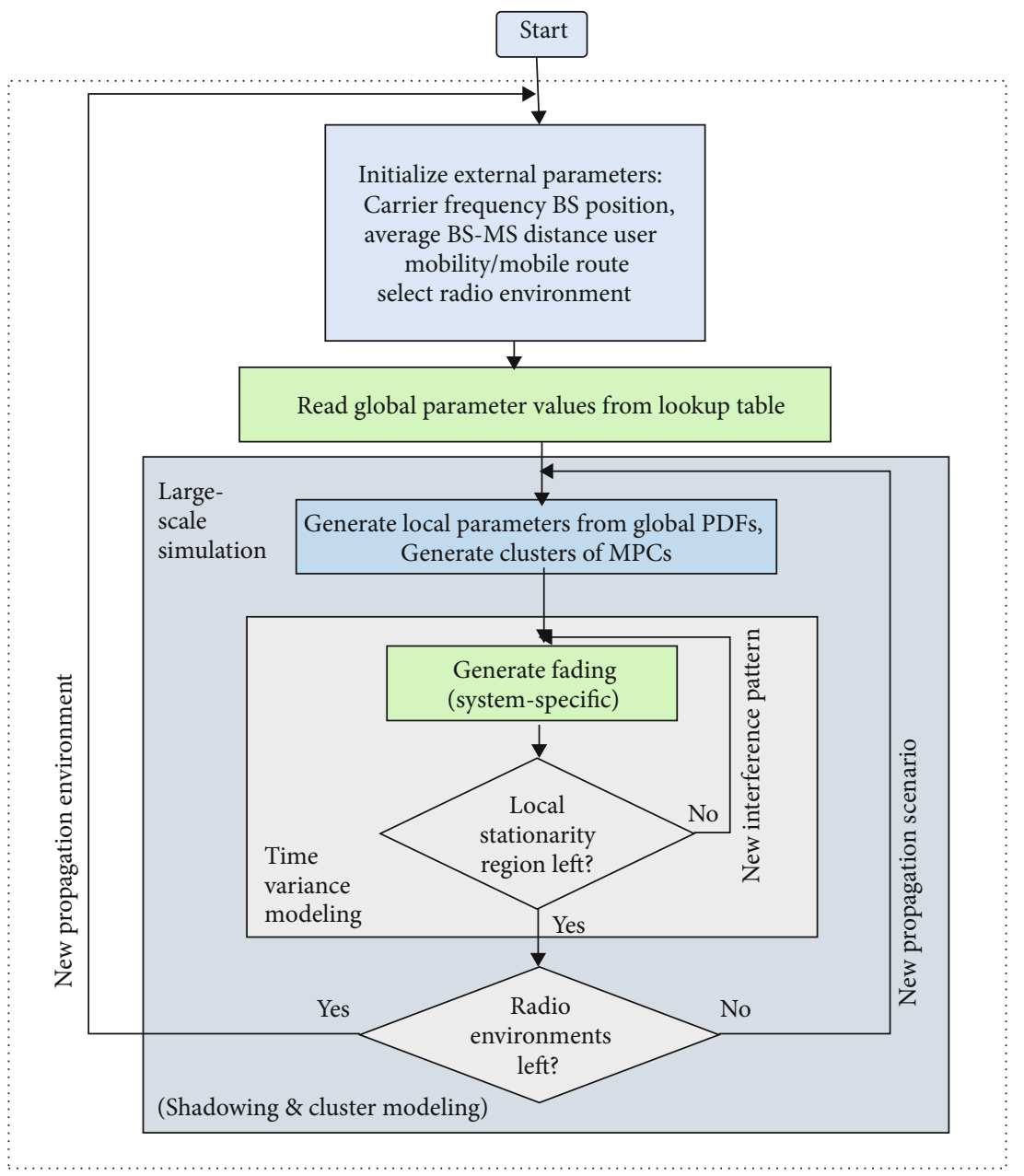

FIgURE 7: Flowchart showing the implementation of the COST 259 directional channel model.

some REs, the LoS and NLoS conditions may occur, following a stochastic pattern.

Global parameters (GPs) help describe the propagation conditions of the entire RE and the number of visible interacting object (IO) clusters defined by a Poisson distribution. In this scenario, the MS within a region of visibility (VR) follows a Poisson process. Accordingly, the GP can be retrieved using ray-tracing techniques, and the associated RE GPs could monitor propagation scenarios.

Last, the local parameters (LPs) are worth mentioning. These are random parameter realizations of the instantaneous channel conditions in a local site. As described in the COST 259 DCM second-layer structure (see Figure 6), LPs' statistical data are given via the collection of GPs [159]. Finally, GPs and LPs are dependent on external parameters, and the flowchart for the model's implementation is shown in Figure 7.

5.4. The COST 273 Model. One of the key models developed by the COST action project is the COST 273 model, and this is the result of an extensive coordinated effort in the European scientific and industrial communities to develop a comprehensive channel model for use in the development of radio communication standards $[108,164]$. A comprehen- sive report on the full framework and implementation of the model for a large urban macrocell environment is presented in [61].

The COST 273 framework's primary focus is on a statistical model for the amplitude, phase, AoA, AoD, and delay of all multipath components. The COST 273, a refinement of the COST 259 model, applies the generic channel model (GCM). The COST 273 model extends the GCM to the case where multipath components can be clustered, depending on the environment for which the model is defined, whether macrocell, microcell, or picocell, the transmitter and receiver can move to different locations $[165,166]$. The most determining factor is the COST 273 environment, the $\mathrm{Tx}$ and $\mathrm{Rx}$ location, including the surrounding buildings' density. It is worthy of note that each environment has different parameters, and the set of all parameters is classified into external and stochastic parameters. On the one hand, the external parameters are user-defined and are maintained constant for a simulation run. On the other hand, the stochastic parameters provide the information required to generate all random variables in the model.

Three types of clusters have been defined in this model [167]. These include the single-interaction clusters, which simulate the scenario when there is a strong correlation 


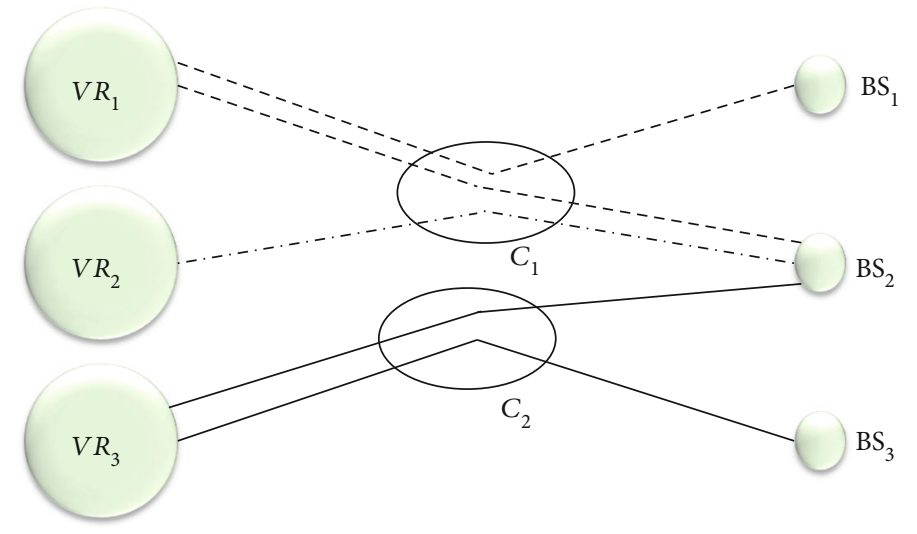

FIgure 8: A simple illustration of the common clusters associated with the COST 273 channel model. A VR must not see more than one cluster, and $\mathrm{Ci}$ denotes clusters that can associate with more than one BS and VR.

between the AoA and AoD statistics. A practical example is when the BS and the MS illuminate the same set of scatterers. Second, local clusters comprise two single-interaction clusters. In this scenario, one cluster is located at the transmitter and the other at the receiver. Third, twin clusters simulate multiple-bounce scattering, where there is rich NLoS scattering between the receiver and transmitter. Figure 8 gives the common clusters' basic concept, consistent with the COST 273 model [55]. Just like any other channel model, the COST 273 has its strengths and limitations.

5.4.1. Strengths. The COST 273 model, compared with the COST 259 double-directional channel model, lends itself to physical interpretations and broad applicability. First, the parameters of the model hold relatively straightforward physical interpretations. This implies that specific parameters of the model can be changed to depict different environments and the effects under study, even without actual measurements campaign. Second, when the parameters are derived from measured data, they show useful information, revealing the investigated environments' characteristics, such as the density of scatterers around each link end. Third, the model has general features making it suitable for applications in several environments.

5.4.2. Limitations. The disadvantages of the COST 273 model circle around complexity, complex stochastic parameters, model specificity, and the inability to implement all aspects of the model. First, the model has so many working parts, and most of these functional parts change rapidly from one environment to another. Keeping track of all the parameters and implementing a general program to accommodate all environments can be computationally expensive. Second, it can be very tasking to derive some of the model's stochastic parameters from measured data, partly due to their sensitivity to calibration errors. For instance, a great deal of care is required in angular power measurements when calibrating for power and antenna gain in the azimuth and elevation. In practice, this can be very difficult due to the dynamic nature of the environment.

However, the model's elevation parameters can be relaxed to simplify the model to ease computation efforts.
Third, the model lacks reliable specifics and does not accurately represent any single channel but instead describes many related environments' average behavior. This can pose a severe problem for a designer who wants to reproduce a single channel, such as a specific path between two buildings in a particular environment. Finally, the model's parameter list is not exhaustive, and it is challenging to implement all aspects of the model [165]. An extension to the COST 273 model is reported in [168].

5.5. The Random Cluster Model. Considering the vast limitations of the COST 273 model, the need to refine the model becomes imperative. In [169], a cluster-based model known as the random cluster model (RCM) was proposed. This model was developed based on a refinement of the existing COST 273 model. The random cluster model presents two characteristics that differentiate it from the COST 273 model. First, the model is capable of automatic identification of cluster parameters from measured data. Second, the identified clusters are characterized using a multivariate PDF called the environment PDF [108].

In contrast with the COST 273 model, RCM targets automatic and faster means of identifying and characterizing clusters from measured data. Analogous to the COST 273 model, the RCM is a system-level cluster model, suitable for evaluating algorithmic and transceiver design trade-offs in specific environments. RCM characterizes each environment using the environment PDF by changing the environment probability density function (PDF). This is unlike the COST 273 model that varies from one environment to the next. This is why the implementation complexity of the RCM is low when compared with the COST 273 model. The RCM model computes the environment PDF automatically from measured data, resulting in a model closely aligned with the investigated environment and measurement routes. This feature makes it very hard to include the model in a standard as the specifics of the environment PDF makes it difficult to describe the average behavior of many channels in a related environment [170].

Another key feature of the RCM is introducing a smoothly time-varying cluster birth-death process to simulate movement. A joint clustering and tracking framework 


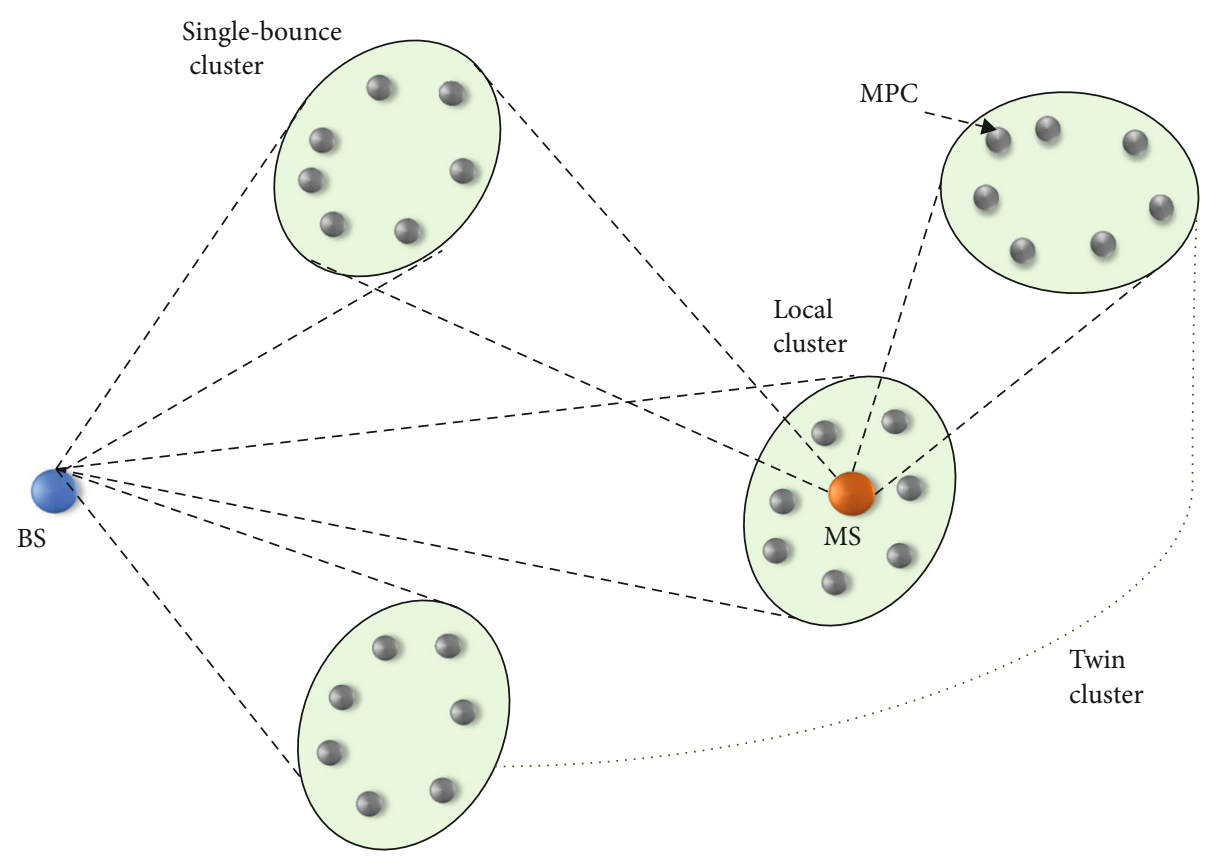

FIGURE 9: A simple structure of the COST 2100 channel model showing the mobile station, base station, single-bounce cluster, local clusters, twin clusters, and multipath components.

has been adopted to compute the cluster movement parameters from experimental data automatically. Just like any other channel model, the RCM model has its strengths and limitations outlined as follows.

5.5.1. Strengths. The advantages of the RCM include time variation, double direction, system independence, and simplicity. First, the RCM focuses on tracking clusters over time, resulting in an accurate channel time variation simulation. Just like the COST 273 model, the RCM is a double-directional model. Similar to the COST 273 model, the RCM is system-independent and models the effects of MPCs independently. Last, it is relatively simple to produce exemplar channels once the environmental PDF has been computed.

5.5.2. Limitations. The computation of environmental PDF is quite a difficult task. Also, several parameters in the clustering and tracking framework affect how the measurement paths are clustered. In this model, the path parameters are specific to the measured path, and the simulation channel will be specific to that particular path. This makes it very difficult to vary the given environment parameters to obtain simulation results for a given environment. Also, the indeterminate number of parameters is worth noting. With every snapshot, it is observed that the total number of parameters in the model changes correspondingly. There is a direct interaction between the clusters' size and the channel's total number of parameters. This means more clusters, more paths, and more significant clusters require more paths to be correctly defined, as remarked in [108].

5.6. COST 2100 MIMO Channel Model. The COST 2100 channel took its root from the COST 259 and COST 273 models [171]. This model can mimic the stochastic properties of multilink MIMO channels over the trio: time, frequency, and space. It is generic and versatile compared to other GSCM models. This makes it suitable for applications relevant to the simulation of multiuser MIMO systems. The basic structure of the COST 2100 model is shown in Figure 9. The model is an extension to the COST 273 model by focusing on the following: the expansion of multicell MIMO scenarios, the multipath contribution polarization model, and the inclusion of dense multipath component specular contribution. Besides, the model defines three ways for the entire set as follows. First, a clear description of the large number of clusters equipped with well-defined stochastic parameters in the simulation relative to the BS's positioning is outlined. Second, identify and derive the scattering of the visible clusters at each channel case. Third, processing large-scale parameters (LSPs) is focused on cluster scattering reported in [172].

In this channel model, the LSP statistics are guaranteed in each channel sequence of instances, which is a vital strength of the model. However, some drawbacks arise from forcing the statistical accuracy of the LSPs. This relates that the model lacks the desired level of flexibility since it does not endorse continuous channel definitions over intervals more significant than the autocorrelation distance. Thus, this distorts the simulation of large MS movements. Secondly, the model dictates that the propagation environment is defined only from the LSPs. The implication is that the inclusion of new LSPs would require a redefinition of the entire initialization of the site, which will result in a distortion in the extension of the model. Next, we take a close look at the model's structure, cluster parameterization, modeling concepts, model developments, parameterization, and implementation. 

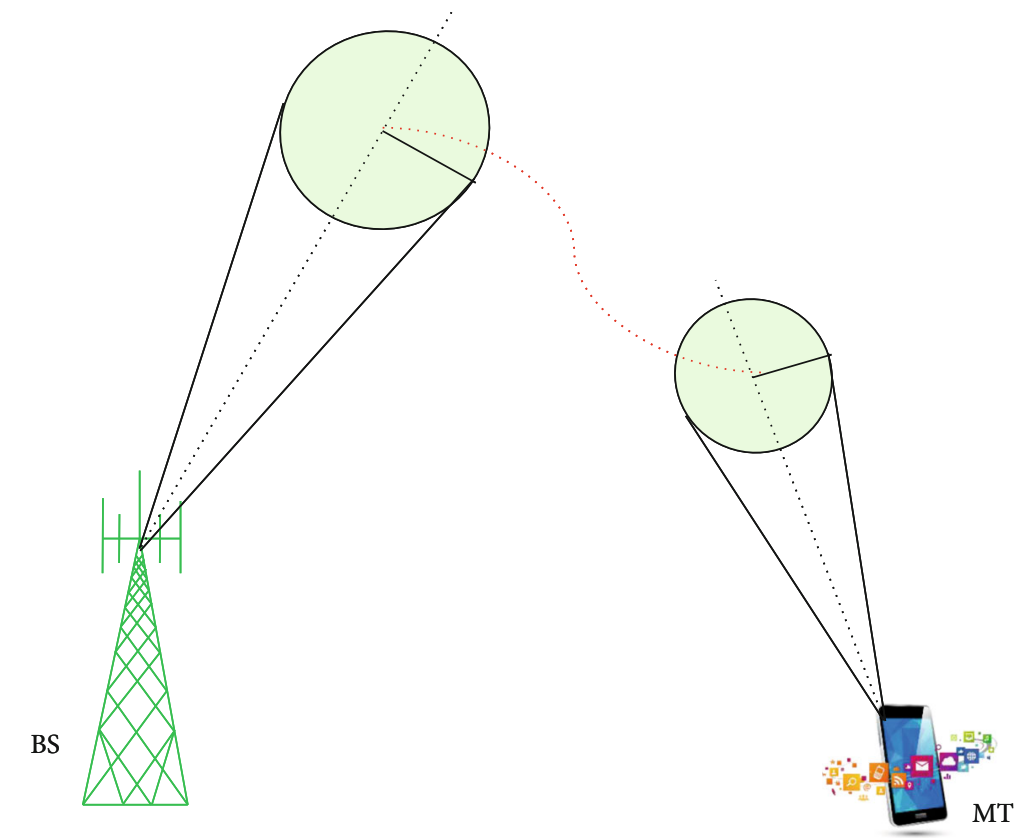

FIGURE 10: A simple illustration depicting the rotation of the twin cluster at the mobile terminal (MT) and the base station (BS) side.

5.6.1. Structure of the COST 2100 Channel Model. This model was developed to replicate the propagation channel between the BS multiple static antennae and the MS multiple antennae [173]. The structure of the COST 2100 channel model is correlated with local, single, and twin-cluster bounces. The local clusters are clustered around the MS or BS, distinguished only by their single-bounce scatterers. This indicates that in the azimuth plane, the local clusters have omnidirectional distribution and their delay and spread of elevation achieve their spatial distribution. Further to this, the singlebounce clusters have a different delay and azimuth spreads than the local clusters [171]. Another cluster variant worth mentioning is the far cluster. This is a mixture of clusters of single and multiple bounces. By matching their delays and angles via geometrical analysis, single-bounce clusters could be mapped to a specific location. The multiple-bounce clusters are represented by their views from the BS and the MS sides, respectively.

This is, in practice, known as twin clusters [95]. Far clusters are distributed all over the simulation environment, having an average density that is consistent with the Poisson distribution. Here, the average number of active state clusters monitors a specific channel's LSPs. This means that the clusters are visible to the MS in the active state, thus contributing to the channel [108]. In reality, the visibility region (VR) definition can be used to know whether there are far-flung clusters accessible [174]. This definition helps to confine the cluster's operation to a given boundary in a geographical site [175]. The twin cluster ratio to the single-bounce cluster is constant in a particular environment [176]. A simple illustration of the twin cluster rotation at the MT and the transmitter end is shown in Figure 10. In [108], the twin-cluster idea was used to suggest a technique for modeling multiple interactions of MPCs with dynamic objects in the BS-MS paths [177]. A standard example of a geometry-based stochastic channel model showing how black dots within each cluster reflects the individual multipath components is shown in Figure 11 [173]. The concept of the model is designed to allow easy adaptation to different environments. Additional information on the interdependence of the MIMO cluster parameters can be found in the literature $[176,178,179]$.

5.6.2. Cluster Parameterization. There is a need for cluster parameterization in channel modeling. Clustering paths helps to define the LSPs of the channel better. These include the delay of the cluster-link, the delay and angular spread of the MPCs in each cluster, the cluster's attenuation, and the level of random shadowing. Whenever the excess delay of the cluster increases, there is an exponential increase in cluster attenuation. Typically, uncorrelated clustering is presumed in most cases. This implies that the LSPs of several clusters are statistically independent. Practical LSP values for the macro-, micro-, and picocellular scenarios are presented in [173].

5.6.3. COST 2100 Modeling Concepts. Visibility regions, clusters, line of sight, multipath elements, and time evolution are primary modeling concepts in the COST 2100 channel model. These principles of modeling are briefly defined as follows.

Visibility regions: a circular region of the simulation area given a fixed scale is the visibility region (VR). A VR specifies only one cluster's visibility. Visibility regions confine the behaviors of clusters within a defined geographic area. In practice, the associated cluster smoothly increases its visibility when MS enters within a VR. A factor named VR gain is used to account for VR. This factor increases from zero to one as the MS gains entry into the VR.

Furthermore, if the MS is at a location in a region where multiple VRs tend to overlap, the effect is the simultaneous 


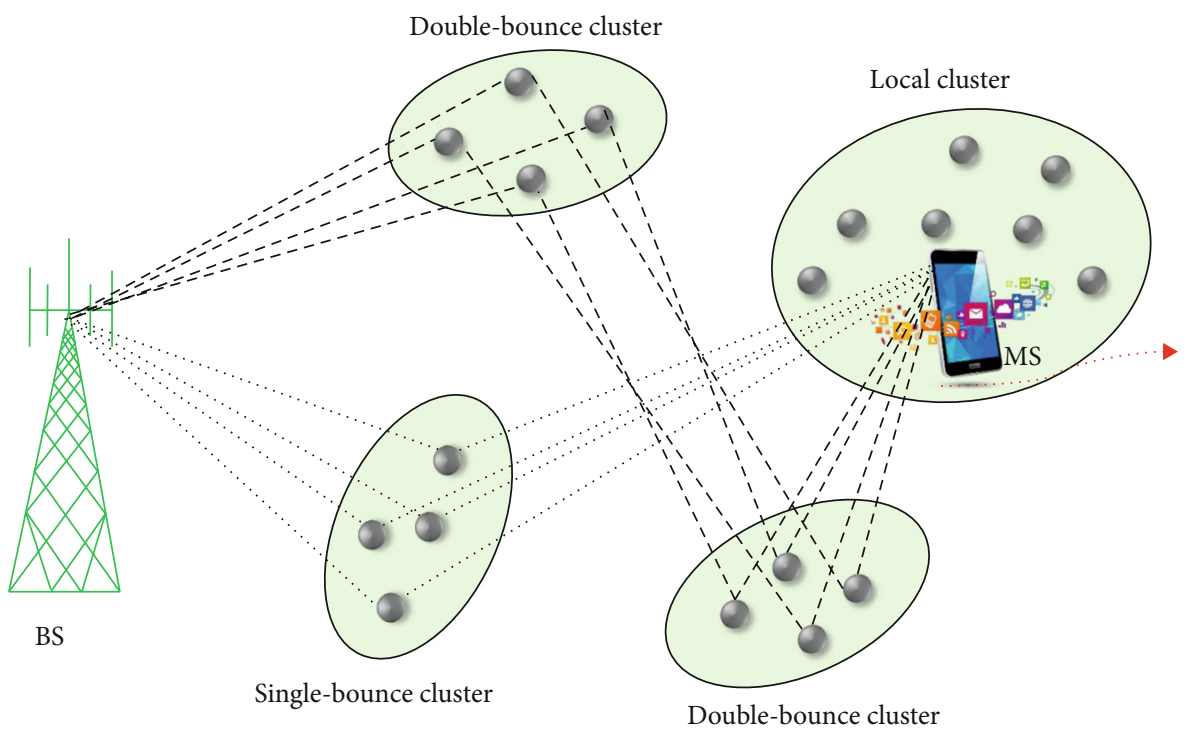

Figure 11: A typical example of a geometry-based stochastic channel model. The black dots inside each cluster represent the individual multipath components.

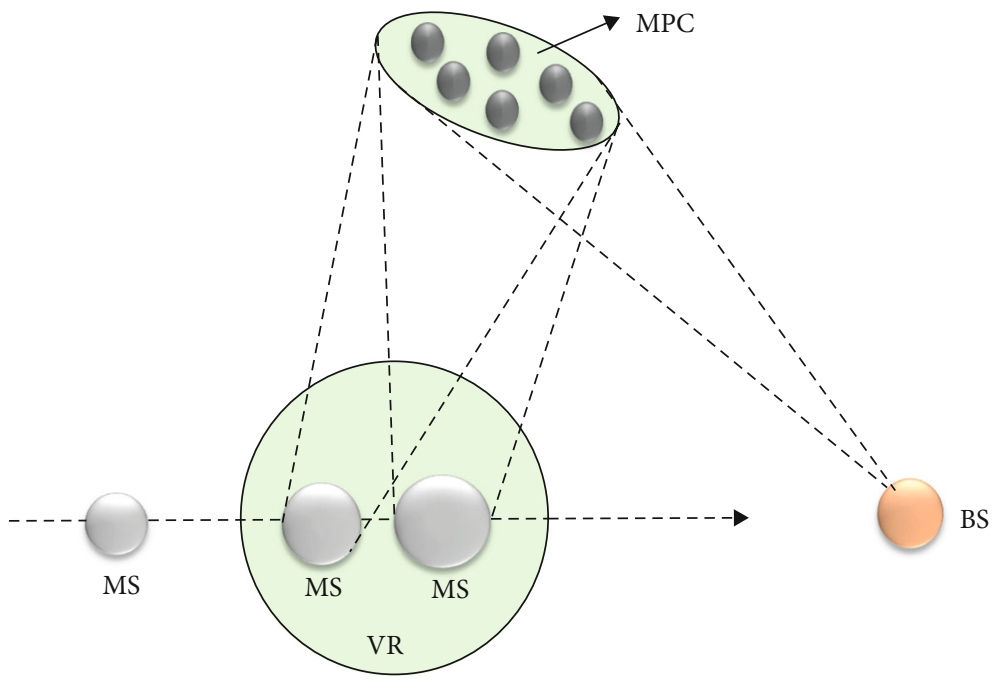

FIGURE 12: Concept of visibility region. BS: base station; MPC: multipath component; MS: mobile station; VR: visibility region.

visibility of multiple clusters. The COST 2100 channel model practically observes a uniform distribution of VRs in and around the simulation region. In this scenario, the VR density is closely aligned to the average number of visible clusters derived from an extensive measurement campaign. As illustrated in Figure 12, after the MS passes through the VR, the MS receives signals dispersed by the corresponding cluster. As it reaches the VR center, the cluster steadily increases its visibility [180]. Mathematically, when going into the VR, a factor called VR gain is used to account for visibility, and it rises from 0 to 1 . Here, the cluster evolution on the receiver side can be defined using the VR size and gain, as noted in [181]. A simple illustration of the concept of cluster visibility regions extended to the BS side. Each of the antenna signs denoting a small MIMO array is shown in Figure 13.

Time evolution of the COST 2100 model: regardless of the MS location, the environment consisting of the clusters and

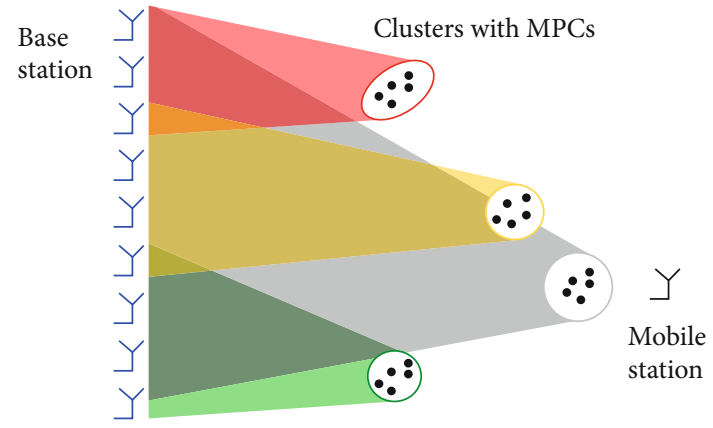

FIGURE 13: A simple illustration of cluster visibility regions extended to the BS side, and each of the antenna sign denotes a small MIMO antenna array. 


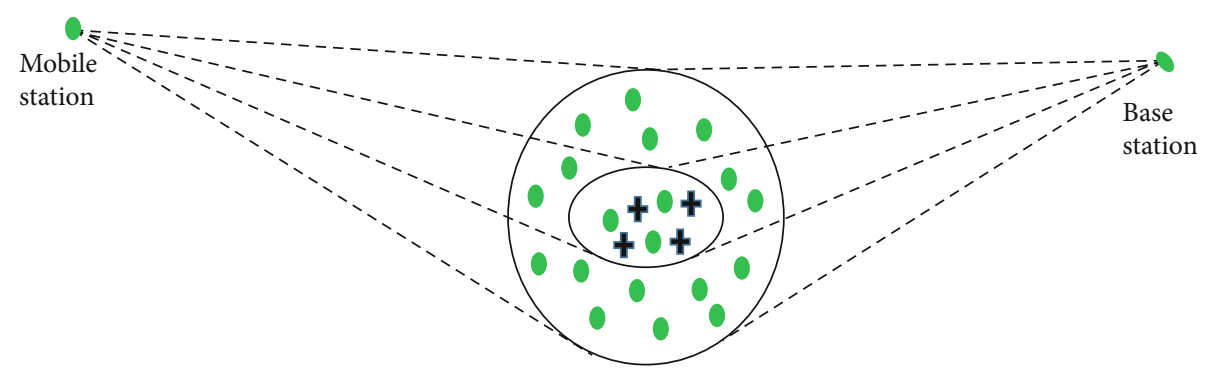

+ Specular MPC

- DMC

Figure 14: A simple description of cluster-based diffused multipath components (DMC).

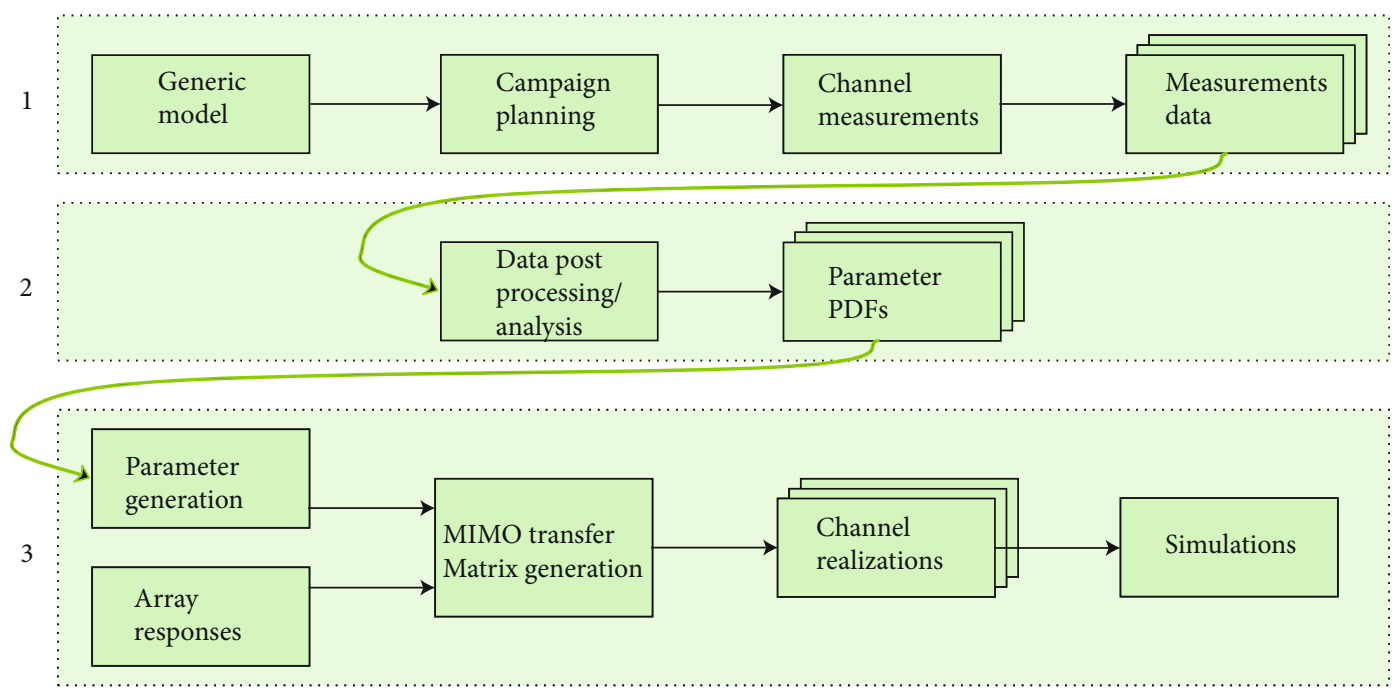

FIGURE 15: Block diagram representation of the procedure for channel coefficient generation for the WINNER channel model.

VRs can be created independently, and virtual sites are created similarly. These conditions are capable of reproducing, in a stochastic manner, the characteristics of an actual site, the exact position, and shapes of scatterers, clusters, and their VRs. Furthermore, the COST 2100 model's layout and parameterization do not depend on the MS speed. The higher the speed, the quicker the MS travels to and fro the VRs, reducing the stationary channel distance. This indicates that the COST 2100 channel model is very fit for the simulation of high-speed MS scenarios.

5.6.4. COST 2100 Developments. Recent developments leading to the extension of the model include but are not limited to polarization, dense multipath components, and multilink aspects. These are briefly described as follows.

Polarization: at the cluster level, the polarization characteristics of the channel are clarified. An MPC is composed of four polarization components $[171,182]$. These polarizations are given as follows: (1) horizontal to horizontal (HH), (2) vertical to vertical (VV), (3) vertical to horizontal (VH), and (4) horizontal to vertical (HV). Besides, a power matrix tends to characterize the power ratios among the given polarization components of each MPC.
Dense multipath components: experimental findings reported in [171] show that specific parameters such as corner diffractions, rough reflections of surfaces, and reflections from a scatterer's different layers result in a significant amount of diffuse scatter. Therefore, an inference is drawn that diffuse scattering can significantly affect the channel's behavior in both delays and angular domains, which may not be adequately captured by a few specular MPCs. To this end, two methods were established for using diffuse scattering.

First, the propagation path can be extended to satisfy diffuse scattering characteristics following a continuous distribution in the delay and angular domains, depending on path dispersion modeling's efficiency. Second, it is possible to superimpose a reasonably large number of specular paths modified with dense multipath components (DMCs). However, this method would increase the whole parameters, especially with large numbers of DMCs, which can give an excellent capture of the residual channel spectrum at the same time [173]. The second approach is regarded in the COST 2100 model as an extension of the MPC definition. A subgroup of DMCs increases the delay and angular spectra from each MPC within the cluster, whose powers decrease in the delay, and angular domains, concerning the MPC. Notice 


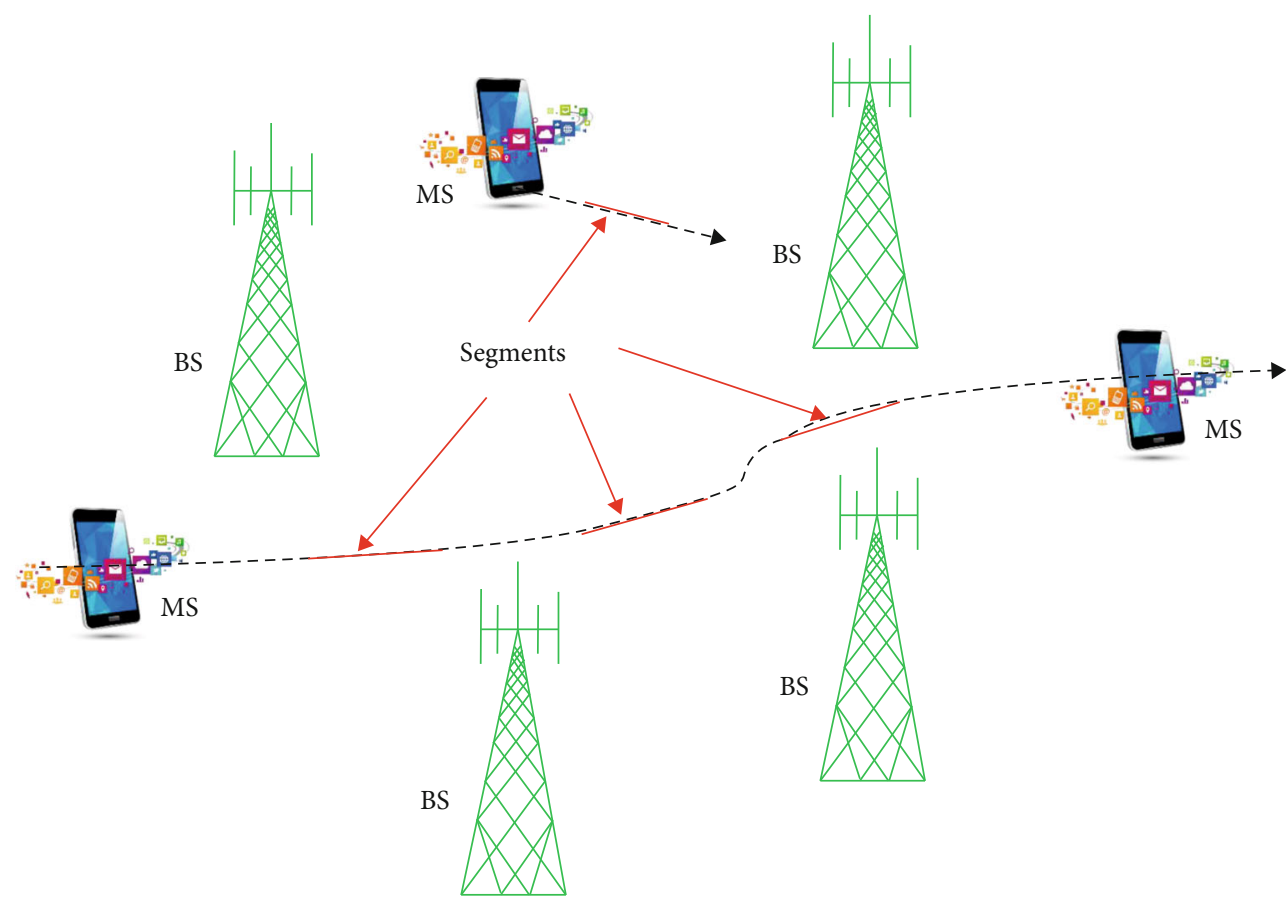

FIGURE 16: Illustration of the WINNER II multilink channel model showing the base stations, mobile terminals, and segments.

TABLE 3: Comparison of the WINNER II, WINNER+, and 3GPP 3D channel models.

\begin{tabular}{|c|c|c|c|}
\hline Parameter & WINNER II & WINNER+ & 3GPP channel model \\
\hline Scenarios & $\begin{array}{l}\text { Urban, macro, and micro outdoor to } \\
\text { indoor, indoor, indoor to outdoor, }\end{array}$ & $\begin{array}{l}\text { Suburban, urban, indoor, macro, and } \\
\text { micro outdoor to indoor scenarios }\end{array}$ & $\begin{array}{l}\text { Urban, outdoor, macro, and } \\
\text { micro outdoor to indoor }\end{array}$ \\
\hline Outdoor height of eNB & $\begin{array}{l}25 \mathrm{~m} \text { and } 10 \mathrm{~m} \text { for macro and } \\
\text { micro, respectively }\end{array}$ & $\begin{array}{c}25 \mathrm{~m} \text { and } 10 \mathrm{~m} \text { for macro and micro, } \\
\text { respectively }\end{array}$ & $\begin{array}{l}25 \mathrm{~m} \text { and } 10 \mathrm{~m} \text { for macro } \\
\text { and micro, respectively }\end{array}$ \\
\hline $\begin{array}{l}\text { Indoor height of user } \\
\text { equipment }\end{array}$ & $\begin{array}{l}1.5 \mathrm{~m} \text { to } 7.5 \mathrm{~m} \text { for three-floor } \\
\text { scenario }\end{array}$ & $1.5 \mathrm{~m}$ to $7.5 \mathrm{~m}$ for three-floor scenario & $\begin{array}{l}1.5 \mathrm{~m} \text { to } 22.5 \mathrm{~m} \text { for eight-floor } \\
\text { scenario }\end{array}$ \\
\hline Line of sight probability & Height independent & Height independent & Depends on height \\
\hline Path loss & Height-gain nonexistent & Models height-gain & Models height-gain \\
\hline $\begin{array}{l}\text { Power angular spectrum } \\
\text { (zenith) }\end{array}$ & Models Gaussian & Models Laplacian & Models Laplacian \\
\hline $\begin{array}{l}\text { Mean zenith angle spread } \\
\text { of departure }\end{array}$ & It is constant & It is constant & Depends on distance and height \\
\hline $\begin{array}{l}\text { Zenith angle of departure } \\
\text { offset }\end{array}$ & It is not modeled & It is constant & Depends on distance and height \\
\hline
\end{tabular}

that the DMC shares the cluster's large-scale properties, such as attenuation of shadowing and cluster control. The overview of cluster-based DMC showing how DMC clustering is superimposed at the same centroid to multipath component clustering is shown in Figure 14.

Multilink aspects: multilink aspects explore the concurrent communications that are spatially isolated between several BSs and MSs. By definition, multiuser scenarios correspond to the single connection in the COST 2100 model. This is due to its ability to accurately characterize one BS's environment regardless of the position where the MS is located.

Various connections can be associated via correlation in a situation where the BSs and MSs are reasonably separated by distance. This concept is described as correlated clustering; clusters exhibit correlated fading or LSPs in separate ties
$[183,184]$. Another way to understand this idea is to consider that clusters are simultaneously visible in various connections. This implies that between multiple ties, clusters are common. This approach's main requirement is to define cluster visibility in various linkages without altering other cluster's physical properties. This will result in a wellmatched extension of the COST 2100 model for single-link scenarios to the current structure. The commonness of cluster connection is an auxiliary function of clusters in multilink systems, and it does not affect cluster definition coverage in terms of power attenuation and spatial distribution. To improve the consistency between the individual connection channels in multilink scenarios, proper modeling of the cluster connection's stochastic properties' commonness becomes imperative. 
TABLE 4: A brief description of the SUI and IEEE 802.xx channel models.

\begin{tabular}{|c|c|c|}
\hline S/N. & Channel models & Brief description \\
\hline 1 & $\begin{array}{l}\text { Stanford University Interim } \\
\text { channel models }\end{array}$ & $\begin{array}{l}\text { Towards the development of the SUI model by researchers at Stanford University, IEEE } 802.16 \\
\text { provided specifics for fixed wireless access (FWA) systems [200]. The group worked on frequency } \\
\text { bands below } 11 \mathrm{GHz} \text { after establishing guidelines for frequency bands above } 11 \mathrm{GHz} \text {. The SUI } \\
\text { models are classified into terrain types A, B, and C. In particular, terrain type A deals with huge path } \\
\text { loss, and it is most suited for hilly terrain with densities of moderate to heavy foliage. Type C is } \\
\text { closely related to minimal path loss, and it is specific to flat terrain with densities of light trees. } \\
\text { Specifically, type B can be suitably applied to hilly terrains with light tree densities or mostly flat } \\
\text { terrains with moderate to heavy tree densities. The path loss equation and the correction factors } \\
\text { relevant to the SUI model are given in [200]. Six SUI tap-delay lines are presented in the SUI channel } \\
\text { models, with three taps valid for a distance of } 7 \text { km between the transmitter and the receiver. For } \\
\text { channels } 1 \text { to } 4 \text { of the SUI model, Ricean is distributed as the first tap, and Rayleigh fading } \\
\text { characterizes the others. Another prominent feature of the SUI models is that a rounded shape } \\
\text { centered very close to zero is given to each tap's Doppler spectrum, and this has limited information } \\
\text { in the Jakes spectrum [126]. }\end{array}$ \\
\hline 2 & IEEE $802.11 \mathrm{n}$ channel models & $\begin{array}{l}\text { The IEEE } 802.11 \mathrm{n} \text { is a simplified form of the extended SV model. This set of models is carefully built } \\
\text { with bandwidths up to } 100 \mathrm{MHz} \text { [201], and the model finds useful applications in indoor MIMO } \\
\text { LAN networks at } 2 \mathrm{GHz} \text { and } 5 \mathrm{GHz} \text {. Here, six canonical channels are modeled systematically to } \\
\text { cover flat fading situations, residential, traditional workplace, large office, small office, and large open } \\
\text { spaces. MATLAB implementation of the model is given in [202]. }\end{array}$ \\
\hline 3 & IEEE 802.16 a channel models & $\begin{array}{l}\text { Following the modification to the popular SUI channel models, which find useful applications in } \\
\text { directional and omnidirectional antennas, the IEEE } 802.16 \text { models were derived. The IEEE } 802.16 \\
\text { models use directional antennas to increase the Ricean taps' K-factor and decrease the spread of } \\
\text { global delay. One key advantage of the IEEE } 802.16 \text { a model is that it does not alter the user terminal's } \\
\text { correlations when reducing the antenna beamwidth. This is contrary to the assumption that as the } \\
\text { beamwidth decreases, the correlation coefficients will increase. Further to this, the IEEE } 802.16 \mathrm{a} \\
\text { includes a path loss model, an appropriate model depicting the Ricean K-factor narrowband, and an } \\
\text { antenna gain reduction factor model. Here, three terrain categories are included in the path loss } \\
\text { model. These are hilly terrain with moderate-to-heavy tree densities as category A, category B as } \\
\text { terrain with intermediate path loss state, and category C is mostly flat terrain with light tree densities. } \\
\text { Category A would find useful applications in models } 5 \text { and } 6 \text { of the SUI, category B for models } 3 \text { and } \\
4 \text { of the SUI, and category C for models } 1 \text { and } 2 \text { of the SUI [28]. A typical example of a spatial channel } \\
\text { model is derived based on this standard [99]. }\end{array}$ \\
\hline 4 & IEEE $802.16 \mathrm{~d} / \mathrm{e}$ channel models & $\begin{array}{l}\text { The IEEE } 802.16 \mathrm{~d} / \mathrm{e} \text { models are an updated version of the interim SUI channel model intended for } \\
\text { fixed macrocellular connectivity. The model is true for the directional and omnidirectional antennas, } \\
\text { which contribute to an increase in the global } K \text {-factor, while the distribution of delays tends to } \\
\text { decrease. The log-normal model of shadowing path loss forms the basis of the IEEE } 802.16 \mathrm{~d} \text {. Three } \\
\text { types of model categorizations (type A, type B, and type C) are derived. These depend on the density } \\
\text { (e.g., tree densities) of the obstacle separating the transmitter from the receiver, considering a } \\
\text { microsuburban environment. The MIMO channel models in the IEEE 802.16e standard have been } \\
\text { established in the WiMAX Forum, following several reports [203-206]. }\end{array}$ \\
\hline
\end{tabular}

5.6.5. Parameterization and Implementation. Propagation measurements have been reported on parameterization for the COST 2100 channel model [11]. These measurements give valuable data, which are required for the parameterization of the model. The model describes the stochastic parameters for relations between (1) VR and cluster, (2) VR, LoS, and cluster powers, (3) cluster shadowing and MPC fading, (4) locations of VR, cluster, LoS, and MPCs, (5) locations, powers, and fading of DMC, and (6) field polarization. In the work of $[171,173]$, a full list of model parameters and suggested distributions for the COST 2100 model is presented. The model implementation requires a complete picture of the propagation site and the MIMO channel matrix synthesis, coupled with the doubledirectional channel and the transmitter and receiver steering vectors of the antenna. Implementing the DMCs is comparable to the MPCs, where each MPC can be projected onto its polarization matrix to obtain the multipolarized subchannels. Finally, the model implementation is well discussed in [11].

Model validation: model validation focuses on angular spread and delay spread, distributions of parameters, and interlink correlation. A comparative study of propagation measurements and simulations on the interlink connection in a dual BS and single MS was used in $[11,171]$ to test the model's validity. Measurements have also been recorded in an indoor $5.3 \mathrm{GHz}$ corridor. Here, the BS and MS are considered vertically polarized planar dipole antenna arrays. Descriptions of the validation results are provided in [55]. Furthermore, as reported in $[75,181]$, the COST 2100 was extended for 3D channel modeling [185], polarization, physically wide array, and closely spaced users. Finally, in terms of modeling and validation, massive MIMO extensions to the COST 2100 channel model are given by [186]. 


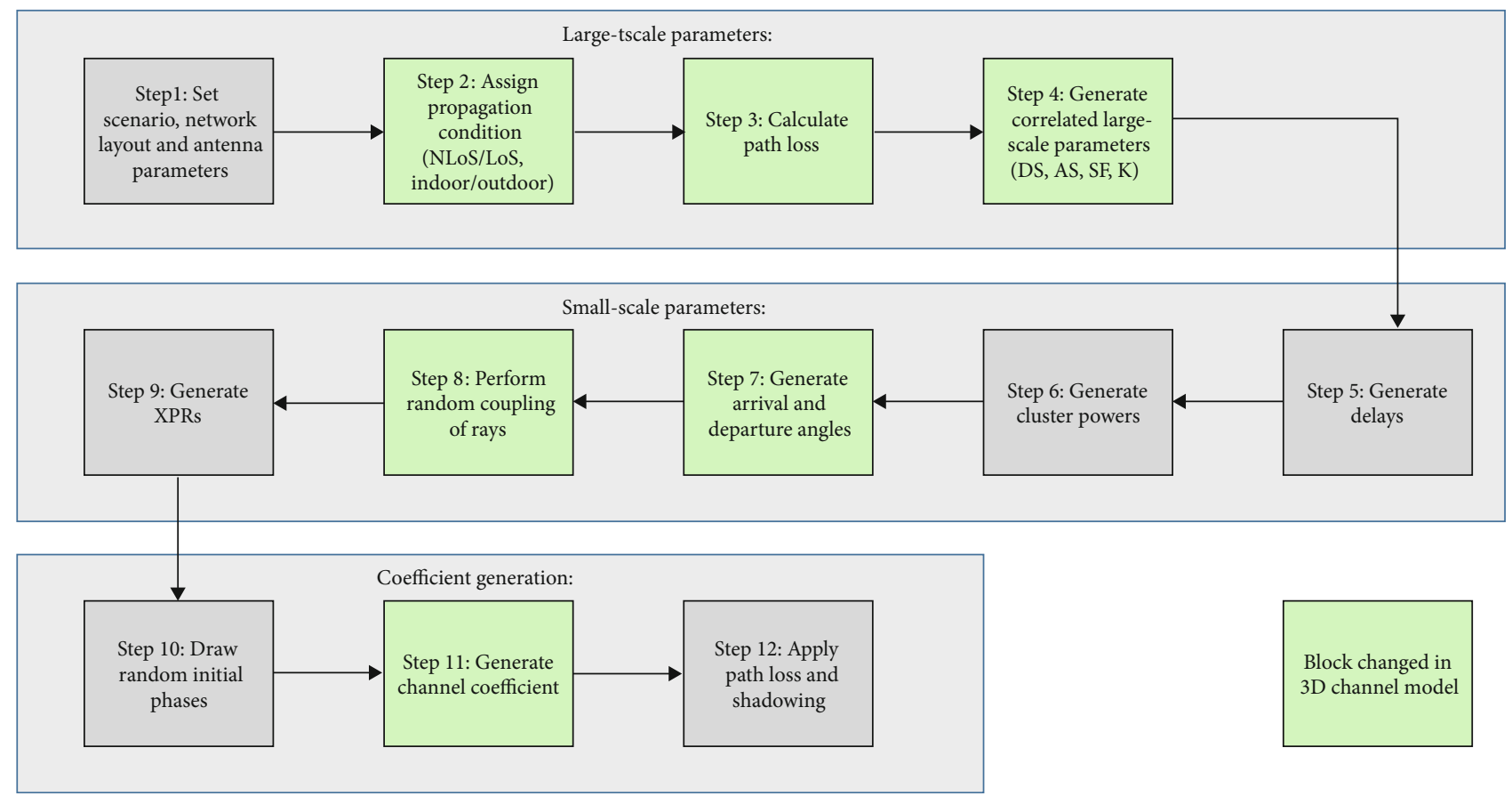

FIGURE 17: Block diagram illustrating the steps for channel generation in the 3GPP 3D model.

\subsection{WINNER Channel Models}

5.7.1. Interim WINNER Channel Model. Considering the minimal parameters available in the 3GPP/3GPP2 SCM model, the interim WINNER model expanded the SCM to include the intracluster delay spreads, LoS, and $K$-factor models, for all scenarios. The implementation was achieved by first specifying a range of large-scale parameters such as the Ricean K-factor, spreading delay, shadowing standard deviation, and spreading directions at both departure and arrival. The model follows a well-defined distribution pattern for a particular connection to fix the large-scale parameters given in [187].

Furthermore, it can be inferred from the WINNER channel model that successive channel matrices can be created in short segments, and a sample of large-scale parameters corresponds to those short segments [188]. In a situation where two segments appear to be quite similar, the clusters are produced independently for each segment. In this case, both segments share large-scale parameters that are strongly correlated but see different clusters [189]. Like the COST 2100 model, the WINNER model produces multipaths for any segment, using clusters and scatterers [190, 191]. For the WINNER model, a block diagram representing the channel coefficient generation procedure is given in Figure 15. A detailed report on model validation is given in [192]. A comparative analysis shows the SCM performances and the extended SCM, and the generic WINNER channel model is given in [193].

5.7.2. WINNER II Multilink MIMO Channel. The revision to the 1st WINNER model gave birth to the WINNER II model [188]. As illustrated in Figure 16, the geometry-based sto- chastic model of the multilink method is a standard-level model. For all the radio links between the BS and MS to be realized, the propagation environment should be well specified. In this model, simultaneous stimulation of the multilinks between the BSs and the MTs can be easily recognized. In contrast, in the SCM model, each simulation is run separately for each connection. When parameters of large scale are correlated, the correlation between multiple links can be introduced. This is because correlation is a distance feature as per implemented multisegment scenarios [194]. One of the fascinating aspects of the modified WINNER model is that large-scale statistics can be derived for any realization scenario [52]. However, in each case, the propagation environment's initialization does not link realizations readily, in an independent manner, which is an essential requirement for evaluating the time variations induced by the users' motion. This implies that, at the system level, the model forces this consistency, making the model lacking in flexibility. This presents problems with the basic extension of the WINNER II multilink MIMO channel model. Typical large-scale parameters for this model at $2.53 \mathrm{GHz}$ in an urban macrocell setting are provided in [195]. Following the description in [196-198], the QuaDRiGa project gave an extension to the WINNER II/+ models, and Table 3 provides a comparative analysis of the $3 \mathrm{D}$ channel models: WINNER II, WINNER+, and 3GPP channel model [199].

5.8. SUI and IEEE 802.xx Channel Models. The SUI and the IEEE 802.xx channel models are presented in Table 4. The IEEE 802.xx channel models comprise the IEEE 802.11n channel model, IEEE 802.16a model, and the IEEE $802.16 \mathrm{~d} / \mathrm{e}$ channel models. 
TABLE 5: Description of critical aspects of the 3GPP 3D channel model. S/N. The aspect of 3D
channel model

1. Applicability of the 3D channel model model has validity in the neighborhood of $1.5 \mathrm{~m}$ to $22.5 \mathrm{~m}$ for UE heights. The $1.5 \mathrm{~m}$ height is essentially the level of a street. As reported in $[199,213,214]$, an indoor UE can be linked with a height denoted by $h_{\mathrm{UE}}=3$

$\left(n_{\text {floor }}-1\right)+1.5$, where $n_{\text {floor }}$ refers to the floor number, which has a uniform distribution between 1 and $N_{\text {floor}}$, and $N_{\text {floor }}$ is the building height in floors. More specifically, this is uniformly distributed between 4 and 8 . The height of the outdoor UEs can typically be taken to be $1.5 \mathrm{~m}$. This aspect demonstrates the extension of the current 3GPP and ITU-R specifications, where a street-level UE is normally modeled.

For each antenna elements in the $2 \mathrm{D}$ channel model, corresponding channel responses are generated in the 3D channel model, among other details. It is worthy to note that $3 \mathrm{D}$ channel modeling helps address some significant limitations of the 2D channel model.

The polarization is another parameter worth noting. It is a crucial feature required in the modeling of antennas. Suppose the cross-polarized transmit antenna pair of $\pm 45 / 45$ degrees is considered. In that case, the constant

2. Antenna modeling polarization model tends to bring about an equal power split for all UE positions in the vertical and horizontal directions. On the other hand, equal power split at the antenna boresight in both the vertical and horizontal directions is seen via the slanted dipole polarization model. However, the power split ratio depends on the UE position in both azimuths and elevation measurements. It can be inferred that the antenna patterns of the simulated eNBs affect the use of a particular polarization model in any simulation scenario.

Line of sight likelihood and path loss are primary parameters often considered in MIMO channel modeling. The effect on LoS likelihood and path loss modeling of various UE heights ranging from $1.5 \mathrm{~m}$ to $22.5 \mathrm{~m}$ for the 3DUMa and 3D-UMi scenarios has been documented [199] [215]. Simulation of the likelihood of LoS, path loss,

3. LoS probability and
path loss modeling and the fast fading channel's characterization requires the inclusion of the channel model's building dimensions. In 3GPP, a decision was reached to adopt the stochastic modeling method used in the SCM and WINNER II, independent entirely, on the building/street orientation dimensions. In the development of the 3D-UMa and $3 \mathrm{D}-\mathrm{UMi}$, some of the $2 \mathrm{D}$ channel model parameters were used. This results in enormous savings in propagation measurement costs and reduces computational complexity for simulations at the machine level [199].

In $3 \mathrm{GPP}$, the LoS path modeling is achieved by applying the 3D distance separating the eNB from the UE. The coefficients for 3D-UMa and 3D-UMi by using LoS path loss equations were specified in the ITU working

4. Path loss modeling documents. For the modeling of NLoS path loss, in a 3D-UMa environment, the dominant propagation paths appear to move over rooftops via multiple diffraction, by diffraction occurring at the edge of a building, following (LoS and NLoS) the report in [214]. Measured data recorded by 3GPP [214] shows that the path loss increases with the diffraction angle as the UE moves from a high floor towards a low floor. A linear term for the height-gain expressed as $\alpha(h-1.5)$ is implemented for functional modeling, and $\alpha(\mathrm{dB} / \mathrm{m})$ is referred to as the gain coefficient.

For a cellular downlink, fast fading can be represented such that the AoA and the AoD are specified on the UE side and eNB side, respectively. The time variance of wireless channels coordinated by a mix of multipath and UE motion is modeled by fast fading channel coefficients.

Further information on fast fading is available in the 3GPP recommendation document [199, 214], explaining how the radio channels are formed. As shown in Figure 16, the channel realizations followed a hierarchical protocol. It is worth noting that there is no description of the propagation between the first and the last interaction. This method could aid in modeling several interactions with the scattering media. For example, this is an indication that the geometry cannot achieve the delay of multipath. Parameters of arrival and departure have to be exchanged for the uplink case, and presumption is made for the downlink scenario. The channel coefficient generation process from steps 4 to 11 for the LoS O2I scenario is the same for the NLoS scenario illustrated in Figure 16.

In general, the channel generation equation is correctly updated in the last step of the channel generation coefficient to account for the ZoAs and ZoDs. A structure for studying 3D channel model extensions and features is given in [216], and preliminary results on 3D channel modeling are presented in [217]. Further to this, the reports in $[218,219]$ also include implementing, validating, and deploying the open-source simulation software model. 
TABLe 6: Parameters for the transitioning from 2D to 3D channel modeling.

\begin{tabular}{|c|c|c|}
\hline S/N. & Parameters & Description \\
\hline 1 & 3D UE dropping & $\begin{array}{l}\text { Earlier channel models specified that the UE is at } 1.5 \mathrm{~m} \text { in height. In this case, the propagation distance } \\
\text { determines the elevation characteristics of the direction of the LoS. The closer the UE to the BS, the higher } \\
\text { the elevation, relative to the direction of the LoS. As a result, users experiencing related slow fading channel } \\
\text { conditions would display closely related elevation in the direction of the LoS, and it will be challenging to } \\
\text { distinguish vertically. Fortunately, TSG-RAN WG1 [214] has given a 3D UE drop to ease the vast } \\
\text { limitations. The latest UE drop allows users to be outside in only } 20 \text { percent of the cases, where their height } \\
\text { is maintained at } 1.5 \mathrm{~m} \text {. }\end{array}$ \\
\hline 2 & $\begin{array}{l}\text { Outdoor to indoor users } \\
\text { for UMa and UMi }\end{array}$ & $\begin{array}{l}\text { According to ITU's modeling specifications, the outdoor to indoor relationship demonstrates a } \\
\text { propagation mode specified in the urban-microcell scenario in [213] to help UEs located within buildings. } \\
\text { The latest 3GPP channel model has expanded this to UMa as well. }\end{array}$ \\
\hline 3 & High-rise scenario & $\begin{array}{l}\text { The high-rise case was suggested to characterize the UE channel on very high floors (more than } 20 \text { ), } \\
\text { following the report [214]. Also, indoor distributed antenna systems can be implemented to assist users in } \\
\text { such buildings. Still, this solution cannot always be extended to high residences, and improved results have } \\
\text { been suggested for uptilt beams. }\end{array}$ \\
\hline 4 & $\begin{array}{l}\text { A new expression for } \\
\text { the path loss }\end{array}$ & $\begin{array}{l}\text { The path loss is dependent on the frequency of transmission, the distance of the UE from the BS, and the } \\
\text { heights of the antennas used for radio signal transmission and reception. Following the results of } \\
\text { comprehensive measurements, the working group, TSG-RAN-WG1 [214], updated the ITU channel } \\
\text { model's path loss expression to accommodate UE height's effects in the NLoS scenario. Finally, the path } \\
\text { loss expression used a 3D distance to replace the 2D distance. }\end{array}$ \\
\hline 5 & $\begin{array}{l}\text { Distance-dependent } \\
\text { large-scale parameters }\end{array}$ & $\begin{array}{c}\text { At the meeting, 3GPP [214] suggested modeling elevation characteristics as lognormal random variables } \\
\text { with specified parameters for UMa scenarios. }\end{array}$ \\
\hline 6 & $\begin{array}{l}\text { The policy used for } \\
\text { UE attachment }\end{array}$ & $\begin{array}{l}\text { In addition to estimating the path loss for the reference signal received power (RSRP), an agreement was } \\
\text { also reached at the meeting to account for fast fading. The RSRP estimation depends only on slow fading } \\
\text { components and path loss before this decision [214]. }\end{array}$ \\
\hline
\end{tabular}

\section{3D Channel Modeling}

Two key performance indicators of great significance in wireless communication systems are the link's SE and reliability [207, 208]. The current 2-dimensional (2D) channel models presented in [209] from the SCM, ITU, and WINNER II are essential candidates for channel modeling and characterization. However, these models cannot capture the elevation's characteristics, and as such, their applications are limited to the azimuth dimension. Thus, there is a need for channel models capable of 3D applications to create and assess multiple antenna techniques involving elevation characteristics [210, 211].

3 GPP recently reported a 3 -dimensional (3D) channel model $[212,213]$ to accommodate the $2 \mathrm{D}$ channel model limitations. Leveraging on the degree of freedom provided by the $3 \mathrm{D}$ channel, it is easy to align the beamforming potentials for every user at various locations, thus enhancing the signal intensity obtained at the $\mathrm{Rx}$ and mitigating the potential impact of interference with colocated users in the same channel [212].

The 3D channel model development follows the WINNER+/WINNER II paradigm with extensions involving some parameters related to height and distance-dependent elevation. This led to the development of multiantenna transmission techniques using large-scale antenna structures, and the potentials of a MIMO channel's 3D spatial dimensions were also exploited by 3GPP [188].

The $3 \mathrm{D}$ channel model discussed here is a geometrybased stochastic model. COST 259, COST 273, COST 2100, SCM, and WINNER II/+ channel models follow the cluster-based approach and extend the 2D channels from the ITU/WINNER II models. Channel generation steps for the 3GPP 3D model are illustrated in Figure 17. Further information about the 3D channel model applicability, antenna modeling, probability and path loss of LoS, and model fast fading are given in Table 5.

6.1. Transitioning from $2 D$ to $3 D$ Channel Modeling. The transition from $2 \mathrm{D}$ to $3 \mathrm{D}$ channel models has seen remarkable improvements worth noting in this paper. The changes observed include UMa and UMi outdoor to indoor users, 3D UE dropping, distinct path loss expressions, high-rise scenario, large-scale distance-dependent parameters, and UE attachment policy. These are briefly described in Table 6.

\section{Open Research Issues}

There is no doubt that some studies have been carried out in wireless communications, especially as it relates to radio propagation channel modeling in MIMO communication systems. However, there are still open research areas in this field yet to be adequately explored. While this list is not exhaustive, critical areas of current relevance to wireless communications are highlighted briefly in Table 7.

\section{Lessons Learned}

In this survey, several lessons are drawn. These include but not limited to (1) difficulty in the development of generalized propagation channel models, (2) dependency of emerging wireless channel models on earlier channel models, (3) 
TABLE 7: Open research issues.

\begin{tabular}{lll}
\hline $\mathrm{S} / \mathrm{N}$. & Open research issues & Brief explanation
\end{tabular}

First, conducting large-scale channel measurements is a major concern. It is still very difficult to carry out large-scale channel measurements, due partly to the complex nature of the dynamic environment, especially for outdoor scenarios, and the sophisticated equipment that is required for such measurements in the mmWave $[177,220]$ and Terahertz $(\mathrm{THz})$

[221] bands. In the case of double-directional channel measurements, switched channel sounders have been used. To the various antenna elements, and in a sequential manner, the

1. Large-scale measurements at mmWave and Terahertz bands

2. Multilink channel measurements
High-precision measurements equipment

4.

Directional characterization

5. Channel model parameterization

6. Channel elevation characteristics sounding signal produced from a single waveform generator is applied. The received signal measurements follow this in a fashion similar to the transmitted signal, and this measurement type has some inherent limitations. First, radio signals transmitted via cables to the antennas from the waveform generator, usually located several miles away, is costly and quite tasking, contributing to large signal attenuation [222]. Fiber optic cables have been deployed for electrooptical conversion and transmission of signals to solve this problem. However, proper calibration and temporal stability are still crucial issues that require further investigation.

In practice, it can be very tedious to establish critical parameters required for multilink modeling. These parameters include correlation information on the angular spreads and joint cluster identification. Most measurements cannot give the necessary joint doubledirectional evaluations of multiple transmitter and receiver locations. Consequently, system designs requiring such parameters are seriously hampered. There is a need for a comprehensive model to accommodate these key parameters. Multilink channel models often present some difficulties related to modeling the correlation properties between the different links. This is because the different links appear to identify both joint clusters and disjoint clusters. This scenario can be very worrisome due to the challenging correlated shadowing processes. Future research could explore high-reliability techniques to simplify the modeling of the correlation properties of multilink channels.

Mobile devices and applications have witnessed a tremendous surge in usage by millions of subscribers in recent years. This poses the need for large-scale measurements. Larger bandwidth fuels the demand for near precise frequency references, leading to an increase in the amount of data collected, usually in thousands of megabytes. The measurement campaign will most likely continue to be the method of investigating channel models. If reliable information related to the channel is required, the measurement equipment must be well designed with high precision and reliability. Future research could be directed towards more sophisticated, efficient, and high-precision measurement equipment.

Directional characterization is still a major problem in mmWave channels. Although the IEEE 802.15.3c [223] and IEEE 802.11ad [224] models provide useful information on directional characterization, there is still an urgent need for extensive measurement verification in different environments. Future studies could also be directed towards investigating the impact of moving objects and human beings' movement obstructing the LoS on the polarization properties. Also, in mmWave and Terahertz channels, the characterization of temporal variations remains an open issue.

Channel model parameterization is still a critical area that needs to be addressed. Most channel models have been parameterized for easy characterization of the stochastic radio channel responses, especially in multilink MIMO systems with spatial correlation properties [92]. For example, it was reported in [171] that the COST 2100 channel model gives practical results, which include several channel properties for proper modeling. However, this model's extension will require much effort in new field measurements to parameterize adequately and validate the extended model [225]. Future research could also be channeled towards investigating how the various modeling approaches and parameterizations affect the systems' performance and how they can be designed for the most efficient operation in those channels. A better understanding of the propagation channels will provide a very good platform for efficient system designs, to ease the applicability of channel models, especially in outdoor-to-indoor scenarios.

Most findings on elevation characteristics $[226,227]$ of the channel are based on ray-tracing simulations. There is a need for a model, especially for outdoor to indoor links. This will help the mobile station's proper positioning since its height is critical to the studies related to wireless channels' elevation characteristics. Also, there is a need to investigate further how the indoor floor plan and the horizontal positioning of the mobile inside the building impact the channel elevation. A summary of previous research work focusing on 3D modeling, with particular interests in elevation characteristics, is reported [228]. 
TABLE 7: Continued.

\begin{tabular}{|c|c|c|}
\hline $\mathrm{S} / \mathrm{N}$. & Open research issues & Brief explanation \\
\hline 7. & Massive MIMO systems & $\begin{array}{l}\text { Conducting large-scale measurements in massive MIMO systems is still a major issue. This } \\
\text { is because the temporal variability is relatively small, but many antenna elements require } \\
\text { switching. The design of switches for such applications is still a huge issue. It is challenging } \\
\text { to design switches for mmWave frequencies [72, 229]. This is why antennas that require } \\
\text { mechanical forces to be moved or rotated are in use at such frequencies, and this method is } \\
\text { time-consuming and computationally expensive. Future work could explore more efficient } \\
\text { and low-cost techniques for conducting large-scale massive MIMO measurements. }\end{array}$ \\
\hline 8. & Vehicular communications & $\begin{array}{l}\text { Several research works related to vehicle-to-vehicle communications have been reported } \\
\text { [230-235], especially in the developed cities of the world. Critical parameters, such as path } \\
\text { loss and delay spread in different environments, have been reported [236, 237]. The vehicle } \\
\text { types in use and the antennas' positioning call for major concerns [238]. In the first place, } \\
\text { there are several hundreds of heavy-duty trucks and buses plowing the highways, but much } \\
\text { has not been reported about such vehicles. Besides, there is a need to carefully investigate the } \\
\text { impact of such heavy-duty trucks on the transmitters and receivers. We need to know how } \\
\text { the shadowing will be impacted if a big truck or a bus is positioned between the transmitter } \\
\text { and receiver. Also, we need to investigate how cars' parking on the sides of the road could } \\
\text { impact communication systems. Hopefully, these findings will provide viable insights } \\
\text { required for the design of safety-critical applications. }\end{array}$ \\
\hline 9. & $\begin{array}{l}\text { Machine learning-based } \\
\text { channel modeling }\end{array}$ & $\begin{array}{l}\text { The design and development of machine learning-based signal prediction algorithms are } \\
\text { still a significant issue. This is due partly to the lack of a comprehensive dataset for the } \\
\text { development and testing of the models, especially at the Terahertz bands. For example, in } \\
\text { [239], the proposed artificial neural network path loss (PL) model's environmental features } \\
\text { could not give the desired descriptions of the actual propagation characteristics due to } \\
\text { inadequate information about the height. This ultimately affects the accuracy of the } \\
\text { proposed PL prediction models. }\end{array}$ \\
\hline
\end{tabular}

extensive measurement campaign requirements, (4) in-depth knowledge of the wireless propagation environment, and (5) high cost of the design of high-precision measurement equipment. These are briefly discussed as follows.

\subsection{Difficulty in Developing Generalized Channel Models.} This survey has revealed no single channel model fits all environmental conditions. Currently, it is still challenging to include all environmental requirements in one channel model. This is because the radio environment is quite dynamic, and the geographical characterization of one environment is different from another environment due partly to the terrain and structural differences. Toward this end, wireless communication engineers need to improve on the existing design techniques to include the required environmental factors or features to design highly robust channel models for future wireless communication systems.

\subsection{Dependency of Emerging Wireless Channel Models on} Earlier Channel Models. This survey has also revealed that the development of recent and future learning-based predictive channel models would require, to a considerable extent, adequate knowledge of the existing channel models. This implies that a thorough knowledge or deep understanding of the earlier channel models will inevitably develop new learning-based channel models to support future wireless communication systems.

8.3. Extensive Measurement Campaign Is Required for Accurate Model Validation. This survey has shown that an extensive measurement campaign is an essential requirement for channel model validation. It is usually very hard to validate theoretical models without testing the models with real-world propagation measurements. The need for extensive propagation measurements, especially at the higherfrequency bands, is very crucial to proper model validation.

8.4. Deep Knowledge of the Wireless Propagation Environment. Another lesson learned from this survey is that adequate knowledge of the wireless propagation environment is an essential requirement for effective channel modeling. More accurate prediction models are achievable when sufficient information about the investigated environment are included in the model's design.

8.5. High Cost of the Design of High-Precision Measurement Equipment. Another major lesson learned from this survey is that it is costly to obtain high-precision measurement equipment. For example, the design of cutting-edge measurement equipment for the mmWave and Terahertz bands is still a big issue [240]. This is due mainly to the high design cost of such equipment and the high-level research and development requirements. Further research efforts would be required for designing highly efficient and cost-effective measurement equipment.

\section{Conclusion}

An extensive survey on standard radio propagation channel models for MIMO communication systems has been presented in this paper. Starting with the fundamental MIMO channel model, the highlights of critical requirements for 
MIMO channel modeling, channel sounding, and MIMO technology classifications are presented. A general overview of some well-known channel models, which are of vital interest in wireless communication systems, is discussed, emphasizing their strengths and limitations. The models are broadly categorized into physical, analytical, and standardized channel models. The physical models appear to propagate radio signals in double directions, independent of the antenna properties. The analytical models seem to focus more on the impulse response of the channel and antenna characteristics. The standard models often described as reference models are most popular and find practical applications in emerging wireless communication systems. Additionally, the recent $3 \mathrm{D}$ channel model provided by $3 \mathrm{GPP}$ was evaluated, and the transitioning from $2 \mathrm{D}$ to $3 \mathrm{D}$ channel models was highlighted. Finally, a comparative analysis of some emerging channel models was presented. Several open issues and key lessons learned for future research in wireless propagation measurements, channel modeling, and MIMO communication systems are discussed extensively.

\section{Abbreviations}

$\begin{array}{ll}\text { 2D: } & \text { 2-dimensional } \\ \text { 2G: } & \text { Second generation } \\ \text { 3D: } & \text { 3-dimensional } \\ \text { 3G: } & \text { Third generation } \\ \text { 3GPP: } & \text { Third Generation Partnership Project } \\ \text { 4G: } & \text { Fourth generation } \\ \text { 5G: } & \text { Fifth generation } \\ \text { 6G: } & \text { Sixth generation } \\ \text { AoA: } & \text { Azimuth angle of arrival } \\ \text { AoD: } & \text { Azimuth angle of departure } \\ \text { AWGN: } & \text { Additive white Gaussian noise } \\ \text { BS: } & \text { Base station } \\ \text { BU: } & \text { Bad urban } \\ \text { CDMA: } & \text { Code division multiple access } \\ \text { CEPT: } & \text { European Conference of Postal and Telecom- } \\ & \text { munications Administrations } \\ \text { CIR: } & \text { Channel impulse response } \\ \text { COST: } & \text { European Cooperation in Science and } \\ \text { DFT: } & \text { Technology } \\ \text { DL: } & \text { Digital Fourier transform } \\ \text { DMCs: } & \text { Downlink } \\ \text { DoA: } & \text { Diffused multipath components } \\ \text { DoD: } & \text { Direction of arrival } \\ \text { DoF: } & \text { Direction of departure } \\ \text { DS: } & \text { Degree of freedom } \\ \text { EE: } & \text { Delay spread } \\ \text { EMs: } & \text { Energy efficiency } \\ \text { eNB: } & \text { Electromagnetics } \\ \text { ENDF: } & \text { Enhanced node base station } \\ \text { FDB: } & \text { Effective Number of Degree of Freedom } \\ \text { FD-MIMO: } & \text { Full dimensional MiMO } \\ \text { FMM: } & \text { Fast multipole method } \\ \text { FWA: } & \text { Fixed wireless access } \\ \text { GBSMs: } & \text { Geometry-based stochastic models } \\ \text { GCM: } & \text { Generic channel model } \\ & \end{array}$

GPs: $\quad$ Global parameters

GPS: Global Positioning System

HT: Hilly terrain

HV: $\quad$ Horizontal to vertical

i.i.d: $\quad$ Independent and identically distributed

IEEE: Institute of Electrical and Electronics Engineers

IOs: Interacting objects

ITU-R: International Telecommunication UnionRadio

KPI: $\quad$ Key performance indicator

LAN: $\quad$ Local area network

LoS: $\quad$ Line of sight

LPs: $\quad$ Local parameters

LSP: $\quad$ Large-scale parameters

LSR: Local scatterer ratio

LTE: Long-term evolution

LTE-A: LTE-advanced

M2M: $\quad$ Mobile-to-mobile

MIMO: $\quad$ Multiple input multiple output

mMIMO: Massive MIMO

mmWave: Millimetre-wave

MPCs: Multipath components

MS: $\quad$ Mobile station

MT: $\quad$ Mobile terminal

MU-MIMO: Multiuser MIMO

NDF: $\quad$ Number of degree of freedom

NLoS: $\quad$ Nonline of sight

PDDP: $\quad$ Power delay direction profile

PDF: $\quad$ Probability distribution function

PDP: $\quad$ Probability density profile

PS: $\quad$ Propagation scenario

QoS: $\quad$ Quality of service

RA: Rural area

RCM: $\quad$ Random channel model

RE: $\quad$ Radio environment

RSRP: $\quad$ Received signal received power

Rx: Receiver

SCM: $\quad$ Spatial channel model

SE: $\quad$ Spectral efficiency

SFIR: $\quad$ Spatial filtering for interference reduction

SIMO: $\quad$ Single input multiple output

SISO: $\quad$ Single input single output

SNR: $\quad$ Signal to noise ratio

SUI model: Stanford University Interim model

SV model: Saleh-Valenzuela model

THz: $\quad$ Terahertz

TU: Typical urban

Tx: $\quad$ Transmitter

UE: User equipment

UL: Uplink

ULA: Uniform linear array

UMa: Urban macro

UMi: Urban micro

UMTS: Universal Mobile Telecommunications Service

UWB: $\quad$ Ultrawide band

VCR: Virtual channel representation

VH: $\quad$ Vertical to horizontal

VR: $\quad$ Visibility region 


$\begin{array}{ll}\text { WiMAX: } & \text { Worldwide Interoperability for Microwave } \\ & \text { Access } \\ \text { WSSUS: } & \text { Widesense Stationarity Uncorrelated } \\ & \text { Scattering } \\ \text { ZoA: } & \text { Zenith angles of arrival } \\ \text { ZoD: } & \text { Zenith angles of departure. }\end{array}$

\section{Data Availability}

No data were used to support this study.

\section{Conflicts of Interest}

The authors declare that they have no conflicts of interest.

\section{Acknowledgments}

Agbotiname Lucky Imoize is partly supported by a Fulbright Fellowship and the United States International Institute of Education (IIE) Scholarship under Grants E0566501 and PS00246077 as a Visiting Research Scholar at the Wireless@VT Lab, Bradley Department of Electrical and Computer Engineering, Virginia Polytechnic Institute and State University, Blacksburg, VA, USA. We thank Prof. R. Michael Buehrer for his kind support and guidance. This work was carried out in collaboration with the IoT-enabled Smart and Connected Communities (SmartCU) Research Cluster of Covenant University. The Article Processing Charges is sponsored by Covenant University Centre for Research, Innovation, and Development (CUCRID), Covenant University, Ota, Nigeria.

\section{References}

[1] J. O. Ogbebor, A. L. Imoize, and A. A.-A. Atayero, "Energy efficient design techniques in next-generation wireless communication networks: emerging trends and future directions," Wireless Communications and Mobile Computing, vol. 2020, Article ID 7235362, 19 pages, 2020.

[2] O. Alamu, A. Gbenga-Ilori, M. Adelabu, A. Imoize, and O. Ladipo, "Energy efficiency techniques in ultra-dense wireless heterogeneous networks: an overview and outlook," Engineering Science and Technology, an International Journal, vol. 23, no. 6, pp. 1308-1326, 2020.

[3] E. Björnson, J. Hoydis, and L. Sanguinetti, "Massive MIMO networks: spectral, energy, and hardware efficiency," Foundations and Trends in Signal Processing, vol. 11, no. 3-4, pp. 154-655, 2017.

[4] Hien Quoc Ngo, E. G. Larsson, and T. L. Marzetta, "Energy and spectral efficiency of very large multiuser MIMO systems," IEEE Transactions on Communications, vol. 61, no. 4, pp. 1436-1449, 2013.

[5] F. Rusek, D. Persson, B. K. Lau, E. G. Larsson, T. L. Marzetta, and F. Tufvesson, "Scaling up MIMO: opportunities and challenges with very large arrays," IEEE Signal Processing Magazine, vol. 30, no. 1, pp. 40-60, 2013.

[6] T. Marzetta, Massive MIMO: An Introduction, vol. 20, no. 1538-7305/14, 2015Bell Labs Tech. J., 2015.

[7] E. Björnson, E. G. Larsson, and T. L. Marzetta, "Massive MIMO: ten myths and one critical question," IEEE Communications Magazine, vol. 54, no. 2, pp. 114-123, 2016.
[8] T. S. Rappaport, Wireless Communications: Principles and Applications, Prentice Hall, Upper Saddle River, New Jersey, 2nd edition, 2002.

[9] A. F. Molisch, Wireless Communications, John Wiley \& Sons, Inc., 2nd edition, 2012.

[10] A. J. Goldsmith, Wireless Communications, Cambridge University Press, 2005.

[11] M. Zhu, G. Eriksson, and F. Tufvesson, "The COST 2100 channel model: parameterization and validation based on outdoor MIMO measurements at $300 \mathrm{MHz}$," IEEE Transactions on Wireless Communications, vol. 12, no. 2, pp. 888 897, 2013.

[12] M. Zhu, Geometry-Based Radio Channel Characterization and Modeling: Parameterization, Implementation and Validation, Lund University, 2014.

[13] C. Oestges and B. Clerckx, MIMO Wireless Communications: From Real-World Propagation to Space-time Code Design, vol. 17, no. 2-3, 2007Ann. ICRP, 2007.

[14] B. Clerckx and C. Oestges, MIMO Wireless Networks: Channels, Techniques and Standards for Multi-Antenna, MultiUser and Multi-Cell Systems, Academic Press, 2nd edition, 2013.

[15] T. L. Marzetta, E. G. Larsson, H. Yang, and H. Q. Ngo, Fundamentals of Massive MIMO, Cambridge University Press, 2016.

[16] A. Charaf and G. Rodriguez-Guisantes, "Channel models in the near field," in 2017 11th European Conference on Antennas and Propagation, EUCAP 2017, pp. 2861-2865, Paris, France, 2017.

[17] J. Agbinya and M. Masihpour, "Magnetic induction channel models and link budgets: a comparison between two Agbinya-Masihpour models," in IEEE International Conference on Communications and Electronics, pp. 400-405, Nha Trang, Vietnam, 2010.

[18] M. C. Domingo, "Magnetic induction for underwater wireless communication networks," IEEE Transactions on Antennas and Propagation, vol. 60, no. 6, pp. 2929-2939, 2012.

[19] B. Gulbahar and O. B. Akan, "A communication theoretical modeling and analysis of underwater magneto-inductive wireless channels," IEEE Transactions on Wireless Communications, vol. 11, no. 9, pp. 3326-3334, 2012.

[20] C. X. Wang, J. Bian, J. Sun, W. Zhang, and M. Zhang, "A survey of $5 \mathrm{~g}$ channel measurements and models," IEEE Communication Surveys and Tutorials, vol. 20, no. 4, pp. 3142-3168, 2018.

[21] J. Navarro-Ortiz, P. Romero-Diaz, S. Sendra, P. Ameigeiras, J. J. Ramos-Munoz, and J. M. Lopez-Soler, "A survey on 5G usage scenarios and traffic models," IEEE Communication Surveys and Tutorials, vol. 22, no. 2, pp. 905-929, 2020.

[22] F. Wen, H. Wymeersch, B. Peng, W. P. Tay, H. C. So, and D. Yang, "A survey on 5G massive MIMO localization," Digital Signal Processing, vol. 94, pp. 21-28, 2019.

[23] I. Yildirim, A. Uyrus, E. Basar, and I. F. Akyildiz, "Propagation modeling and analysis of reconfigurable intelligent surfaces for indoor and outdoor applications in $6 \mathrm{G}$ wireless systems," pp. 1-10, 2019, https://arxiv.org/abs/1912.07350.

[24] Y. Xing and T. S. Rappaport, "Propagation measurement system and approach at $140 \mathrm{GHz}$-moving to $6 \mathrm{G}$ and above 100 GHz," in 2018 IEEE Glob. Commun. Conf. GLOBECOM 2018 - Proceedings, pp. 1-6, Abu Dhabi, United Arab Emirates, December 2018. 
[25] S. Ju, O. Kanhere, Y. Xing, and T. S. Rappaport, “A millimeter-wave channel simulator NYUSIM with spatial consistency and human blockage," in 2019 IEEE Global Communications Conference (GLOBECOM), pp. 1-6, Waikoloa, HI, USA, 2019.

[26] T. Anggita and M. Suryanegara, "Outdoor to indoor propagation model of glass material building at $26 \mathrm{GHz}$ for $5 \mathrm{G}$ mobile technology," in 2020 8th International Conference on Information and Communication Technology (ICoICT), Yogyakarta, Indonesia, 2020.

[27] A. R. Mishra, Fundamentals of Cellular Network Planning and Optimisation, John Wiley \& Sons, Inc., 2018.

[28] P. Almers, E. Bonek, A. Burr et al., "Survey of channel and radio propagation models for wireless MIMO systems," EURASIP Journal on Wireless Communications and Networking, vol. 2007, no. 1, Article ID 019070, 2007.

[29] A. L. Imoize and O. D. Adegbite, "Measurements-based performance analysis of a $4 \mathrm{G}$ Lte network in and around shopping malls and campus environments in Lagos Nigeria," Arid Zone Journal of Engineering, Technology and Environment, vol. 14, no. 2, pp. 208-225, 2018.

[30] A. L. Imoize, K. Orolu, and A. A.-A. Atayero, "Analysis of key performance indicators of a $4 \mathrm{G}$ LTE network based on experimental data obtained from a densely populated smart city," Data in Brief, vol. 29, no. 105304, article 105304, 2020.

[31] M. Hata, "Empirical formula for propagation loss in land mobile radio services," IEEE Transactions on Vehicular Technology, vol. 29, no. 3, pp. 317-325, 1980.

[32] W. C. Y. Lee, Mobile Communications Engineering, McGrawHill Professional, 1982.

[33] W. C. Y. Lee, Mobile Communications Design Fundamentals, John Wiley \& Sons, Inc., 2010.

[34] J. Walfisch and H. L. Bertoni, "A theoretical model of UHF propagation in urban environments," IEEE Transactions on Antennas and Propagation, vol. 36, no. 12, pp. 1788-1796, 1988.

[35] E. Damasso and L. M. Correia, COST 231-Digital Mobile Radio Towards Future Generation Systems, European Union, 1999.

[36] R. A. Valenzuela, "Antennas and propagation for wireless communications," in IEEE Vehicular Technology Conferenceno. 1, pp. 1-5, Atlanta, GA, USA, 1996.

[37] M. Failli, Digital Land Mobile Radio Communications: Final Report (14 March 1984-13 September 1988), Commission of the European Communities, Directorate-General Telecommunications, Information Industries and Innovation, 1989.

[38] P. S. Jonas Medbo, Channel Models for HIPERLAN/2 in Different Indoor Scenarios, ETSI/BRAN, 1998.

[39] ITU-R, Guidelines for Evaluation of Radio Mobile Activites of ETRI Interface Technologies for IMT Advanced, Vol. 1, International Telecommunication Union (ITU), 2009.

[40] ITUP, Prediction Methods for the Terrestrial Land-Mobile Service Using the Frequency Range $30 \mathrm{MHz}$ to $3 \mathrm{GHz}$, vol. 567-4, CCIR Report, 1995.

[41] J. Scourias, Overview of the Global System for Mobile Communications, Univ. Waterloo, 1995.

[42] M. Tolstrup, Indoor Radio Planning: A Practical Guide for 2G, $3 G$ and 4G, John Wiley \& Sons, Inc., 2015.

[43] J. Laiho, A. Wacker, and T. Novosad, Radio Network Planning and Optimisation for UMTS, John Wiley \& Sons, Inc., New York, USA, 2nd edition, 2002.
[44] H. Holma, J. Melero, J. Vainio, T. Halonen, and J. Mäkinen, "Performance of adaptive multirate (AMR) voice in GSM and WCDMA," in IEEE Vehicular Technology Conference, vol. 57no. 4, pp. 2177-2181, Jeju, South Korea, 2003.

[45] W. Tuttlebee, "Software Defined Radio - Baseband Technology for 3G Handsets and Basestations [Book Review]," Commun. Eng., vol. 2, no. 2, pp. 46-47, 2004.

[46] IEEE Working Group, "IEEE Standard for Information Technology-Telecommunications and information exchange between systems-local and metropolitan area networks," November 2010, https://ieeexplore.ieee.org/xpls/abs_all .jsp?arnumber $=1237563$.

[47] E. Dahlman, S. Parkvall, and J. Sköld, 4G: LTE/LTEAdvanced for Mobile Broadband, Academic Press, 2013.

[48] C. Cox, An Introduction to LTE: LTE, LTE-Advanced, SAE and $4 G$ Mobile Communications, John Wiley \& Sons, Inc., 2012.

[49] A. Elnashar, M. A. El-Saidny, and M. R. Sherif, Design, Deployment and Performance of 4G-LTE Networks: A Practical Approach, John Wiley \& Sons, Inc., 2014.

[50] R. Irmer, H. P. May, A. Weber et al., "Multisite field trial for LTE and advanced concepts," IEEE Communications Magazine, vol. 47, no. 2, pp. 92-98, 2009.

[51] N. Czink, B. Bandemer, G. Vazquez-Vilar, L. Jalloul, C. Oestges, and A. Paulraj, "Spatial separation of multi-user MIMO channels," in IEEE International Symposium on Personal, Indoor and Mobile Radio Communications, PIMRC, pp. 1059-1063, Tokyo, Japan, 2009.

[52] C. Oestges, "Multi-link propagation modeling for beyond next generation wireless," in 2011 Loughborough Antennas \& Propagation Conference, Loughborough, UK, 2011.

[53] C. Oestges, P. Castiglione, and N. Czink, "Empirical modeling of nomadic peer-to-peer networks in office environment," in 2011 IEEE 73rd Vehicular Technology Conference (VTC Spring), Budapest, Hungary, 2011.

[54] S. Salous, S. Feeney, N. Razavi-Ghods, and M. Abdalla, "Sounders for MIMO channel measurements," in 13th European Signal Processing Conference, EUSIPCO 2005, pp. $1963-$ 1966, Antalya, Turkey, 2005.

[55] K. Haneda, J. Poutanen, F. Tuvfesson et al., "Development of multi-link geometry-based stochastic channel models," in 2011 Loughborough Antennas \& Propagation Conference, pp. 1-7, Loughborough, UK, November 2011.

[56] G. Eriksson, F. Tufvesson, and A. F. Molisch, "Propagation channel characteristics for peer-to-peer multiple antenna systems at $300 \mathrm{MHz}$," in IEEE Globecom 2006, San Francisco, CA, USA, 2006.

[57] K. Wiklundh and G. Eriksson, "A study of the capacity for different element spacing on compact MIMO platforms," in 2008 IEEE 19th International Symposium on Personal, Indoor and Mobile Radio Communications, Cannes, France, 2008.

[58] J. Kim, E. A. Lee, C. S. Kim, Y. J. Chong, and J. H. Cho, “An efficient calibration of MIMO channel sounders with internal crosstalk," IEEE Transactions on Vehicular Technology, vol. 69, no. 9, pp. 9445-9458, 2020.

[59] J. Sun, H. Chang, X. Gao, C. X. Wang, and J. Huang, "Multifrequency multi-scenario millimeter wave MIMO channel measurements and modeling for B5G wireless communication systems," IEEE Journal on Selected Areas in Communications, vol. 38, no. 9, pp. 2010-2025, 2020. 
[60] A. Grundinger, M. Joham, and W. Utschick, "Stochastic transceiver design in point-to-point MIMO channels with imperfect CSI," in 2011 Int. ITG Work. Smart Antennas, WSA 2011, Aachen, Germany, May 2011.

[61] L. M. Correia, "COST 273 - towards mobile broadband multimedia networks," in Twelfth International Conference on Antennas and Propagation (ICAP 2003), pp. 569-572, Exeter, United Kingdom, 2003.

[62] J. G. Andrews, S. Buzzi, W. Choi et al., "What will 5G be?," IEEE Journal on Selected Areas in Communications, vol. 32, no. 6, pp. 1065-1082, 2014.

[63] G. Caire and S. Shamai, "On the achievable throughput of a multiantenna Gaussian broadcast channel," IEEE Transactions on Information Theory, vol. 49, no. 7, pp. 1691-1706, 2003.

[64] S. Vishwanath, N. Jindal, and A. Goldsmith, "Duality, achievable rates, and sum-rate capacity of Gaussian MIMO broadcast channels," IEEE Transactions on Information Theory, vol. 49, no. 10, pp. 2658-2668, 2003.

[65] D. Gesbert, M. Kountouris, R. W. Heath, C. B. Chae, and T. Sälzer, "Shifting the MIMO paradigm," IEEE Signal Processing Magazine, vol. 24, no. 5, pp. 36-46, 2007.

[66] G. Caire, N. Jindal, M. Kobayashi, and N. Ravindran, "Multiuser MIMO achievable rates with downlink training and channel state feedback," IEEE Transactions on Information Theory, vol. 56, no. 6, pp. 2845-2866, 2010.

[67] N. Ravindran and N. Jindal, "Multi-user diversity vs. accurate channel state information in MIMO downlink channels," IEEE Transactions on Wireless Communications, vol. 11, no. 9, pp. 3037-3046, 2012.

[68] B. Badic, A. F. Cattoni, M. Dieudonne et al., Advances in Carrier Aggregation and Multi-User MIMO for LTE-Advanced: Outcomes from SAMURAI Project, White paper, 2012.

[69] Q. H. Spencer, C. B. Peel, A. L. Swindlehurst, and M. Haardt, "An introduction to the multi-user MIMO downlink," IEEE Communications Magazine, vol. 42, no. 10, pp. 60-67, 2004.

[70] M. H. M. Costa, "Writing on dirty paper (Corresp.)," IEEE Transactions on Information Theory, vol. 29, no. 3, pp. 439441, 1983.

[71] T. L. Marzetta, "How much training is required for multiuser MIMO?," in Conference Record - Asilomar Conference on Signals, Systems and Computers, pp. 359-363, Pacific Grove, CA, USA, 2006

[72] F. Boccardi, R. Heath, A. Lozano, T. L. Marzetta, and P. Popovski, "Five disruptive technology directions for 5G," IEEE Communications Magazine, vol. 52, no. 2, pp. 74-80, 2014.

[73] E. G. Larsson, O. Edfors, F. Tufvesson, and T. L. Marzetta, "Massive MIMO for next generation wireless systems," IEEE Communications Magazine, vol. 52, no. 2, pp. 186195, 2014.

[74] L. Lu, G. Y. Li, A. L. Swindlehurst, A. Ashikhmin, and R. Zhang, "An overview of massive MIMO: benefits and challenges," IEEE Journal on Selected Topics in Signal Processing, vol. 8, no. 5, pp. 742-758, 2014.

[75] X. Gao, M. Zhu, F. Tufvesson, F. Rusek, and O. Edfors, "Extension of the COST 2100 channel model for massive MIMO,” 2015, https://lup.lub.lu.se/search/publication/ 5114789.

[76] J. Hoydis, S. Ten Brink, and M. Debbah, "Massive MIMO in the UL/DL of cellular networks: how many antennas do we need?," IEEE Journal on Selected Areas in Communications, vol. 31, no. 2, pp. 160-171, 2013.

[77] K. Zheng, S. Ou, and X. Yin, "Massive MIMO channel models: a survey," International Journal of Antennas and Propagation, vol. 2014, Article ID 848071, 10 pages, 2014.

[78] S. Ojo, A. Imoize, and D. Alienyi, "Radial basis function neural network path loss prediction model for LTE networks in multitransmitter signal propagation environments," International Journal of Communication Systems, vol. 34, no. 3, pp. 1-26, 2021.

[79] P. Almers, F. Tufvesson, and A. F. Molisch, "Keyhole effect in MIMO wireless channels: measurements and theory," IEEE Transactions on Wireless Communications, vol. 5, no. 12, pp. 3596-3604, 2006.

[80] R. Mahey and D. J. Malhotra, "On MIMO channel modeling for the mobile wireless systems," International Journal of Future Generation Communication and Networking, vol. 8, no. 5, pp. 23-38, 2015.

[81] A. J. Goldsmith and L. J. Greenstein, "A measurement-based model for predicting coverage areas of urban microcells," IEEE Journal on Selected Areas in Communications, vol. 11, no. 7, pp. 1013-1023, 1993.

[82] D. Gesbert, H. Bölcskei, D. A. Gore, and A. J. Paulraj, "Outdoor MIMO wireless channels: models and performance prediction," IEEE Transactions on Communications, vol. 50, no. 12, pp. 1926-1934, 2002.

[83] X. Cheng, C. X. Wang, D. I. Laurenson, S. Salous, and A. V. Vasilakos, "New deterministic and stochastic simulation models for non-isotropic scattering mobile-to-mobile Rayleigh fading channels," Wireless Communications and Mobile Computing, vol. 11, no. 7, pp. 829-842, 2011.

[84] Z. Yun, Z. Zhang, and M. F. Iskander, "A ray-tracing method based on the triangular grid approach and application to propagation prediction in urban environments," IEEE Transactions on Antennas and Propagation, vol. 50, no. 5, pp. 750-758, 2002.

[85] R. Wang, S. Sangodoyin, A. F. Molisch, J. Zhang, Y. H. Nam, and J. Lee, "Elevation characteristics of outdoor-to-indoor macrocellular propagation channels," in IEEE Vehicular Technology Conference, Seoul, South Korea, 2014.

[86] K. H. Ng, E. K. Tameh, A. Doufexi, M. Hunukumbure, and A. R. Nix, "Efficient multielement ray tracing with sitespecific comparisons using measured MIMO channel data," IEEE Transactions on Vehicular Technology, vol. 56, no. 3, pp. 1019-1032, 2007.

[87] J. Zeleny, F. Perez-Fontan, and P. Pechac, “Generalized propagation channel model for $2 \mathrm{GHz}$ low elevation links using a ray-tracing method," Radioengineering, vol. 24, no. 4, pp. 1044-1049, 2015.

[88] L. Schumacher, L. T. Berger, J. Ramiro-Moreno, and T. B. Sørensen, "Propagation Characterization and MIMO Channel Modeling for 3G," in Adaptive Antenna Arrays, pp. 377-393, Springer, 2004.

[89] R. S. Thoma, D. Hampicke, A. Richter et al., "Identification of time-variant directional mobile radio channels," IEEE Transactions on Instrumentation and Measurement, vol. 49, no. 2, pp. 357-364, 2000.

[90] M. G. Roozbahani and E. Jedari, "A new statistical wideband spatio-temporal channel model for $5-\mathrm{GHz}$ band WLAN systems [Comments and corrections on]," IEEE Journal on Selected Areas in Communications, vol. 26, no. 7, pp. 1328 1330, 2008. 
[91] C. Tepedelenlioğlu and G. B. Giannakis, "On velocity estimation and correlation properties of narrow-band mobile communication channels," IEEE Transactions on Vehicular Technology, vol. 50, no. 4, pp. 1039-1052, 2001.

[92] X. Cheng, C. X. Wang, H. Wang et al., "Cooperative MIMO channel modeling and multi-link spatial correlation properties," IEEE Journal on Selected Areas in Communications, vol. 30, no. 2, pp. 388-396, 2012.

[93] A. F. Molisch, "A generic model for MIMO wireless propagation channels in macro- and microcells," IEEE Transactions on Signal Processing, vol. 52, no. 1, pp. 61-71, 2004.

[94] A. F. Molisch, "Ultrawideband propagation channels-theory, measurement, and modeling," IEEE Transactions on Vehicular Technology, vol. 54, no. 5, pp. 1528-1545, 2005.

[95] H. Hofstetter, A. F. Molisch, and N. Czink, "A twin-cluster MIMO channel model," in European Space Agency, (Special Publication) ESA SP, Nice, France, 2006.

[96] Jianmin Gong, J. F. Hayes, and M. R. Soleymani, “The effect of antenna physics on fading correlation and the capacity of multielement antenna systems," EEE Transactions on Vehicular Technology, vol. 56, no. 4, pp. 1591-1599, 2007.

[97] A. Abdi and M. Kaveh, "A space-time correlation model for multielement antenna systems in mobile fading channels," IEEE Journal on Selected Areas in Communications, vol. 20, no. 3, pp. 550-560, 2002.

[98] C. Oestges, "Validity of the Kronecker model for MIMO correlated channels," in IEEE Vehicular Technology Conference, vol. 6, pp. 2818-2822, Melbourne, Vic., Australia, 2006.

[99] C. Oestges, V. Erceg, and A. J. Paulraj, “A physical scattering model for MIMO macrocellular broadband wireless channels," IEEE Journal on Selected Areas in Communications, vol. 21, no. 5, pp. 721-729, 2003.

[100] M. Souden, S. Affes, and J. Benesty, "A two-stage approach to estimate the angles of arrival and the angular spreads of locally scattered sources," IEEE Transactions on Signal Processing, vol. 56, no. 5, pp. 1968-1983, 2008.

[101] A. Amar, "The effect of local scattering on the gain and beamwidth of a collaborative beampattern for wireless sensor networks," IEEE Transactions on Wireless Communications, vol. 9, no. 9, pp. 2730-2736, 2010.

[102] C. Oestges, B. Clerckx, D. Vanhoenacker-Janvier, and A. J. Paulraj, "Impact of fading correlations on MIMO communication systems in geometry-based statistical channel models," IEEE Transactions on Wireless Communications, vol. 4, no. 3, pp. 1112-1120, 2005.

[103] G. L. Turin, F. D. Clapp, T. L. Johnston, S. B. Fine, and D. Lavry, "A statistical model of urban multipath propagation," IEEE Transactions on Vehicular Technology, vol. 21, no. 1, pp. 1-9, 1972.

[104] A. A. M. Saleh and R. A. Valenzuela, "A statistical model for indoor multipath propagation," IEEE Journal on Selected Areas in Communications, vol. 5, no. 2, pp. 128-137, 1987.

[105] Z. Sahinoglu, Ultra-Wideband Positioning Systems, Cambridge University Press, 2008.

[106] Q. H. Spencer, B. D. Jeffs, M. A. Jensen, and A. L. Swindlehurst, "Experiments in modeling the space-time indoor wireless communication channel," in IEEE Signal Processing Workshop on Signal Processing Advances in Wireless Communications, SPAWC, pp. 297-300, Paris, France, 1997.
[107] Q. Spencer, M. Rice, B. Jeffs, and M. Jensen, "A statistical model for angle of arrival in indoor multipath propagation," in Adaptive Antennas for Wireless Communications, pp. 4448, Phoenix, AZ, USA, 2009.

[108] N. Costa and S. Haykin, Multiple-Input Multiple-Output Channel Models: Theory and Practice, John Wiley \& Sons, Inc., 2010.

[109] T. Zwick, C. Fischer, and W. Wiesbeck, "A stochastic multipath channel model including path directions for indoor environments," IEEE Journal on Selected Areas in Communications, vol. 20, no. 6, pp. 1178-1192, 2002.

[110] B. D. Van Veen and K. M. Buckley, "Beamforming: a versatile approach to spatial filtering," IEEE ASSP Magazine, vol. 5, no. 2, pp. 4-24, 1988.

[111] M. Debbah and R. R. Müller, "MIMO channel modeling and the principle of maximum entropy," IEEE Transactions on Information Theory, vol. 51, no. 5, pp. 1667-1690, 2005.

[112] H. Özcelik, M. Herdin, W. Weichselberger, J. Wallace, and E. Bonek, "Deficiencies of 'Kronecker' MIMO radio channel model," Electronics, vol. 39, no. 16, pp. 1209-1210, 2003.

[113] M. Lienard, J. Baudet, D. Degardin, and P. Degauque, "Capacity of multi-antenna array systems in tunnel environment," in Vehicular Technology Conference. IEEE 55th Vehicular Technology Conference. VTC Spring 2002 (Cat. No.02CH37367), vol. 2, pp. 552-555, Birmingham, AL, USA, 2002.

[114] D. P. Mcnamara, M. A. Beach, and P. N. Fletcher, "Spatial correlation in indoor MIMO channels," in The 13th IEEE International Symposium on Personal, Indoor and Mobile Radio Communications, Pavilhao Altantico, Lisboa, Portugal, 2002.

[115] J. P. Kermoal, L. Schumacher, K. I. Pedersen, P. E. Mogensen, and F. Frederiksen, "A stochastic MIMO radio channel model with experimental validation," IEEE Journal on Selected Areas in Communications, vol. 20, no. 6, pp. 12111226, 2002.

[116] H. Özcelik, N. Czink, and E. Bonek, "What makes a good MIMO channel model?," in IEEE Vehicular Technology Conference, vol. 61no. 1, pp. 156-160, Stockholm, Sweden, 2005.

[117] W. Weichselberger, M. Herdin, H. Özcelik, and E. Bonek, “A stochastic MIMO channel model with joint correlation of both link ends," IEEE Transactions on Wireless Communications, vol. 5, no. 1, pp. 90-100, 2006.

[118] A. M. Sayeed, "Deconstructing multiantenna fading channels," IEEE Transactions on Signal Processing, vol. 50, no. 10, pp. 2563-2579, 2002.

[119] R. U. Nabar, H. Bölcskei, V. Erceg, D. Gesbert, and A. J. Paulraj, "Performance of multiantenna signaling techniques in the presence of polarization diversity," IEEE Transactions on Signal Processing, vol. 50, no. 10, pp. 2553-2562, 2002.

[120] C. Oestges, B. Clerckx, D. Vanhoenacker-Janvier, and A. J. Paulraj, "Impact of diagonal correlations on MIMO capacity: application to geometrical scattering models," in IEEE Vehicular Technology Conference, vol. 58no. 1, pp. 394-398, Orlando, FL, USA, 2003.

[121] C. Oestges, H. Özcelik, and E. Bonek, "On the practical use of analytical MIMO channel models," in IEEE Antennas and Propagation Society, AP-S International Symposium (Digest), vol. 3B, pp. 406-409, Washington, DC, USA, 2005.

[122] W. Xu, S. A. Zekavat, and H. Tong, "A novel spatially correlated multiuser MIMO channel modeling: impact of surface 
roughness," IEEE Transactions on Antennas and Propagation, vol. 57, no. 8, pp. 2429-2438, 2009.

[123] M. Katzin, "The scattering of electromagnetic waves from rough surfaces," Proceedings of the IEEE, vol. 52, no. 11, pp. 1389-1390, 1964.

[124] N. Costa and S. Haykin, "A novel wideband MIMO channel model and McMaster's wideband MIMO SDR," in Conference Record - 2006 Fortieth Asilomar Conference on Signals, Systems and Computers, pp. 956-960, Pacific Grove, CA, USA, 2006.

[125] C. X. Wang, X. Hong, H. Wu, and W. Xu, "Spatial-temporal correlation properties of the $3 \mathrm{GPP}$ spatial channel model and the Kronecker MIMO channel model," EURASIP Journal on Wireless Communications and Networking, vol. 2007, Article ID 39871, 9 pages, 2007.

[126] W. C. Jakes, Microwave Mobile Communications, WileyIEEE Press, 1994.

[127] S. E. Foo, M. A. Beach, and A. G. Burr, "Wideband outdoor MIMO channel model derived from directional channel measurements at $2 \mathrm{GHz}$," in Proceedings of the 7th International Symposium on Wireless Personal Multimedia Communications (WPMC '04), Abano Terme, Padova, Italy, 2004.

[128] A. G. Burr, "Capacity bounds and estimates for the finite scatterers MIMO wireless channel," IEEE Journal on Selected Areas in Communications, vol. 21, no. 5, pp. 812-818, 2003.

[129] D. A. Gore and A. J. Paulraj, "MIMO antenna subset selection with space-time coding," IEEE Transactions on Signal Processing, vol. 50, no. 10, pp. 2580-2588, 2002.

[130] I. Berenguer and X. Wang, "Space-time coding and signal processing for MIMO communications," Journal of Computer Science and Technology, vol. 18, no. 6, pp. 689-702, 2003.

[131] M. R. Amin and S. D. Trapasiya, "Space time coding scheme for MIMO system-literature survey," Procedia Engineering, vol. 38, pp. 3509-3517, 2012.

[132] A. H. Alqahtani, A. I. Sulyman, and A. Alsanie, "Rateless space time block code for massive MIMO systems," International Journal of Antennas and Propagation, vol. 2014, Article ID 154261, 10 pages, 2014.

[133] G. Caire, P. Elia, and K. R. Kumar, "Space-time coding: an overview," Journal of Communications Software and Systems, vol. 2, no. 3, p. 212, 2017.

[134] B. M. Eldowek, S. M. Abd El-atty, E. S. M. El-Rabaie, and F. E. Abd El-Samie, "3D modeling and analysis of the space-time correlation for $5 \mathrm{G}$ millimeter wave MIMO channels," Wireless Personal Communications, vol. 104, no. 2, pp. 783-799, 2019.

[135] A. S. Y. Poon, R. W. Brodersen, and D. N. C. Tse, "Degrees of freedom in multiple-antenna channels: a signal space approach," IEEE Transactions on Information Theory, vol. 51, no. 2, pp. 523-536, 2005.

[136] M. D. Migliore, "On the role of the number of degrees of freedom of the field in MIMO channels," IEEE Transactions on Antennas and Propagation, vol. 54, no. 2, pp. 620-628, 2006.

[137] J. Xu and R. Janaswamy, "Electromagnetic degrees of freedom in 2-D scattering environments," IEEE Transactions on Antennas and Propagation, vol. 54, no. 12, pp. 3882-3894, 2006.

[138] W. Saad, M. Bennis, and M. Chen, "A vision of $6 \mathrm{G}$ wireless systems: applications, trends, technologies, and open research problems," IEEE Network, vol. 34, no. 3, pp. 134-142, 2020.
[139] E. Damosso, "Action COST 231: a commitment to the transition from GSM to UMTS," in IEEE International Conference on Personal Wireless Communications, pp. 234-238, Bangalore, India, 1994.

[140] E. Damosso and G. De Brito, "COST 231 achievements as a support to the development of UMTS: a look into the future," IEEE Communications Magazine, vol. 34, no. 2, pp. 90-96, 1996.

[141] P. E. Mogensen, K. I. Pedersen, P. Leth-Espensen et al., "Preliminary measurement results from an adaptive antenna array testbed for GSM/UMTS," in Adaptive Antennas for Wireless Communications, pp. 690-694, Phoenix, AZ, USA, 2009.

[142] COST231, Digital Mobile Radio: COST231 View on the Evolution towards Third Generation Systems, Eur. Telecommun., 2005.

[143] P. W. Huish, A Review of the Radio Propagation Work Undertaken under the COST 207 Programme, Propag. Factors Interf. Model. Mob. Radio Syst. IEE Colloq., 1988.

[144] A. L. Imoize and T. E. Ogunfuwa, "Propagation measurements of a 4G LTE network in Lagoon environment," Nigerian Journal of Technological Development, vol. 16, no. 1, pp. 1-9, 2019.

[145] S. O. Ajose and A. L. Imoize, "Propagation measurements and modelling at $1800 \mathrm{MHz}$ in Lagos Nigeria," International Journal of Wireless and Mobile Computing, vol. 6, no. 2, pp. 165-174, 2013.

[146] F. Ikegami, S. Yoshida, T. Takeuchi, and M. Umehira, "Propagation factors controlling mean field strength on urban streets," IEEE Transactions on Antennas and Propagation, vol. 32, no. 8, pp. 822-829, 1984.

[147] D. Har, A. M. Watson, and A. G. Chadney, "Comment on diffraction loss of rooftop-to-street in COST 231-WalfischIkegami model," IEEE Transactions on Vehicular Technology, vol. 48, no. 5, pp. 1451-1452, 1999.

[148] G. Calcev, D. Chizhik, B. Goransson et al., "A wideband spatial channel model for system-wide simulations," IEEE Transactions on Vehicular Technology, vol. 56, no. 2, pp. 389-403, 2007.

[149] D. Reed, J. Smith, A. Rodriguez, and G. Calcev, "Spatial channel models for multi-antenna systems," in IEEE Vehicular Technology Conference, vol. 58, pp. 99-103, Orlando, FL, USA, 2003.

[150] D. S. Baum, J. Hansen, G. Del Galdo, M. Milojevic, J. Salo, and P. Kyösti, "An interim channel model for beyond-3G systems: extending the 3GPP spatial channel model (SCM)," in IEEE Vehicular Technology Conference, vol. 61no. 5, pp. 3132-3136, Stockholm, Sweden, 2005.

[151] J. Salo, G. Del Galdo, J. Salmi et al., "MATLAB implementation of the 3GPP spatial channel model," 3GPP TR25.996, 3rd Generation Partnership Project (3GPP), 2005.

[152] F. Adachi and T. T. Tjhung, "Tapped delay line model for band-limited multipath channel in DS-CDMA mobile radio," Electronics Letters, vol. 37, no. 5, pp. 318-319, 2001.

[153] X. Zhao, J. Kivinen, and P. Vainikainen, "Tapped delay line channel models at $5.3 \mathrm{GHz}$ in indoor environments," in IEEE Vehicular Technology Conference, vol. 1, pp. 1-5, Boston, MA, USA, 2000.

[154] J. Sykora, "Tapped delay line model of linear randomly timevariant WSSUS channel," Electronics Letters, vol. 36, no. 19, pp. 1656-1657, 2000. 
[155] H. Xiao, A. G. Burr, and L. Song, "A time-variant wideband spatial channel model based on the 3GPP model," in IEEE Vehicular Technology Conference, pp. 75-79, Montreal, QC, Canada, 2006.

[156] F. Talebi, Stochastic Multipath Modeling of Wideband Polarized MIMO Channels, University of Notre Dame, 2015.

[157] J. Medbo, M. Riback, and J. E. Berg, "Validation of 3GPP spatial channel model including WINNER wideband extension using measurements," in IEEE Vehicular Technology Conference, pp. 119-123, Montreal, QC, Canada, 2006.

[158] H. Asplund, A. A. Glazunov, A. F. Molisch, K. I. Pedersen, and M. Steinbauer, "The COST 259 directional channel model-part II: macrocells," IEEE Transactions on Wireless Communications, vol. 5, no. 12, pp. 3434-3450, 2006.

[159] A. F. Molisch, H. Asplund, R. Heddergott, M. Steinbauer, and T. Zwick, "The COST259 directional channel model-part I: overview and methodology," IEEE Transactions on Wireless Communications, vol. 5, no. 12, pp. 3421-3433, 2006.

[160] H. Asplund, A. F. Molisch, M. Steinbauer, and N. B. Mehta, "Clustering of scatterers in mobile radio channels - evaluation and modeling in the COST259 directional channel model," in IEEE International Conference on Communications, vol. 2, pp. 901-905, New York, NY, USA, 2002.

[161] D. Lingaiah, "Wireless flexible personalised communications: COST 259: European co-operation in mobile radio reseach," Computer Communications, vol. 26, no. 18, article 2106, 2003.

[162] P. Hoeher, "A statistical discrete-time model for the WSSUS multipath channel," IEEE Transactions on Vehicular Technology, vol. 41, no. 4, pp. 461-468, 1992.

[163] G. Matz, "On non-WSSUS wireless fading channels," IEEE Transactions on Wireless Communications, vol. 4, no. 5, pp. 2465-2478, 2005.

[164] L. Smoczyński, A. S. Gallo, and M. Marciniak, "COST Action 273 at the National Institute of Telecommunications," in International Conference on Transparent Optical Networks, vol. 2, pp. 137-140, Warsaw, Poland, 2003.

[165] I. D. Sirkova, Overview of COST 273 Part II: Parabolic Equation Method Application, Sofia, Bulgaria, 2006.

[166] I. Sousa, M. P. Queluz, and A. Rodrigues, "Implementation of the COST 273 directional channel model in microcell scenarios," in SIGMAP 2012, WINSYS 2012 - Proceedings of the International Conference on Signal Processing and Multimedia Applications and Wireless Information Networks and Systems, pp. 349-356, Rome, Italy, 2012.

[167] N. Czink and C. Oestges, "The COST 273 MIMO channel model: three kinds of clusters," in IEEE International Symposium on Spread Spectrum Techniques and Applications, pp. 282-286, Bologna, Italy, 2008.

[168] P. Vieira, P. Queluz, and A. Rodrigues, "Clustering of scatterers over an irregular clutter environment: an extension of COST 273 MIMO channel model," in IEEE Vehicular Technology Conference, vol. 38, pp. 824-828, Baltimore, MD, USA, 2007.

[169] N. Czink, E. Bonek, L. Hentilä, J. P. Nuutinen, and J. Ylitalo, "WLC06-2: cluster-based MIMO channel Mmodel parameters extracted from indoor time-variant measurements," in IEEE Globecom, San Francisco, CA, USA, 2006.

[170] N. Czink, X. Yin, H. OZcelik, M. Herdin, E. Bonek, and B. H. Fleury, "Cluster characteristics in a MIMO indoor propagation environment," IEEE Transactions on Wireless Communications, vol. 6, no. 4, pp. 1465-1475, 2007.
[171] L. Liu, C. Oestges, J. Poutanen et al., "The COST 2100 MIMO channel model," IEEE Wireless Communications, vol. 19, no. 6, pp. 92-99, 2012.

[172] R. Verdone and A. Zanella, Pervasive Mobile and Ambient Wireless Communications: COST Action 2100, Springer Science \& Business Media, 2012.

[173] J. Poutanen, K. Haneda, L. Liu, C. Oestges, F. Tufvesson, and P. Vainikainen, "Parameterization of the COST 2100 MIMO channel model in indoor scenarios," in Proceedings of the 5th European Conference on Antennas and Propagation, EUCAP 2011, pp. 3606-3610, Rome, Italy, 2011.

[174] C. F. Lopez and C. X. Wang, "Novel 3-D non-stationary wideband models for massive MIMO channels," IEEE Transactions on Wireless Communications, vol. 17, no. 5, pp. 28932905, 2018.

[175] A. Ali, E. D. Carvalho, and R. W. Heath, "Linear receivers in non-stationary massive MIMO channels with visibility regions," IEEE Wireless Communications Letters, vol. 8, no. 3, pp. 885-888, 2019.

[176] J. Poutanen, K. Haneda, J. Salmi et al., "Significance of common scatterers in multi-link indoor radio wave propagation," in EuCAP 2010 - The 4th European Conference on Antennas and Propagation, pp. 1-5, Barcelona, Spain, 2010.

[177] P. Zhang, B. Yang, H. Wang, and C.-X. Wang, Radio Propagation Measurements and Cluster-Based Analysis for $5 G$ Millimeter-Wave Cellular Systems in Dense Urban Environments, IEEE Trans. Commun., 2020.

[178] N. Czink, E. Bonek, L. Hentilä, P. Kyösti, J. P. Nuutinen, and J. Ylitalo, "The interdependence of cluster parameters in MIMO channel modeling," in European Space Agency, (Special Publication) ESA SP, vol. 626, Nice, France, 2006.

[179] O. Gordon, Z. Dou, A. P. Morales, and S. Shrestha, "Interlink multi-user MIMO channel capacity and the effects of clusters," in 2011 IEEE 3rd International Conference on Communication Software and Networks, ICCSN 2011, pp. 16-20, Xi'an, China, 2011.

[180] Z. Ping, C. Jianqiao, and T. Tian, "An overview of nonstationary property for massive MIMO channel modeling," ZTE Communications, vol. 15, no. 1, pp. 3-7, 2017.

[181] X. Gao, F. Tufvesson, and O. Edfors, "Massive MIMO channels - measurements and models," in Conference Record - Asilomar Conference on Signals, Systems and Computers, pp. 280-284, Pacific Grove, CA, USA, 2013.

[182] C. Guo, F. Liu, S. Chen, C. Feng, and Z. Zeng, “Advances on exploiting polarization in wireless communications: channels, technologies, and applications," IEEE Communication Surveys and Tutorials, vol. 19, no. 1, pp. 125-166, 2017.

[183] A. Forenza, D. J. Love, and R. W. Heath, "Simplified spatial correlation models for clustered MIMO channels with different array configurations," IEEE Transactions on Vehicular Technology, vol. 56, no. 4, pp. 1924-1934, 2007.

[184] R. He, B. Ai, A. F. Molisch et al., "Clustering enabled wireless channel modeling using big data algorithms," IEEE Communications Magazine, vol. 56, no. 5, pp. 177-183, 2018.

[185] S. Ju, Y. Xing, O. Kanhere, and T. S. Rappaport, "3-D statistical indoor channel model for millimeter-wave and subterahertz bands," pp. 1-7, 2020, https://arxiv.org/abs/2009 .12971 .

[186] J. Flordelis, X. Li, O. Edfors, and F. Tufvesson, "Massive MIMO extensions to the COST 2100 channel model: 
modeling and validation," IEEE Transactions on Wireless Communications, vol. 19, no. 1, pp. 380-394, 2020.

[187] Y. S. Cho, J. Kim, W. Y. Yang, and C. G. Kang, MIMO-OFDM Wireless Communications with MATLAB ${ }^{\circledR}$, John Wiley \& Sons, Inc., 2010.

[188] P. Kyösti, J. Meinilä, L. Hentilä et al., IST-4-027756 WINNER II D1.1.2 Part II Channel Models Radio Channel Measurement and Analysis Results, v1. 0. Tech. rep. WINNER II IST project, 2007.

[189] S. L. H. Nguyen, K. Haneda, J. Järveläineny, A. Karttuneny, and J. Putkonen, "Large-scale parameters of spatiotemporal short-range indoor backhaul channels at 140 GHz," 2020, https://arxiv.org/abs/2009.13209.

[190] M. Narandzic, M. Kaske, C. Schneider et al., "3D-antenna array model for IST-WINNER channel simulations," in IEEE Vehicular Technology Conference, pp. 319-323, Dublin, Ireland, 2007.

[191] M. Narandzic, C. Schneider, and R. S. Thomä, "WINNER wideband MIMO system-level channel model-comparison with other reference models," 2008, https://www .researchgate.net/publication/43941009.

[192] P. Kyösti, D. Laselva, T. Jämsä, and L. Hentilä, Validating IST-WINNER Indoor MIMO Radio Channel Model ITU-R WP5D IMT-2020 Channel Models View Project OTA Testing View Project Validating IST-WINNER Indoor MIMO Radio Channel Model, IST Mobile and Wireless Summit, Mykonos, Greece, 2006.

[193] M. Narandžić, C. Schneider, R. Thomä, T. Jämsä, P. Kyösti, and X. Zhao, "Comparison of SCM, SCME, and WINNER channel models," in IEEE Vehicular Technology Conference, pp. 413-417, Dublin, Ireland, 2007.

[194] M. Döttling, W. Mohr, and A. Osseiran, Radio Technologies and Concepts for IMT-Advanced, John Wiley \& Sons, Inc., 2010.

[195] C. Schneider, M. Narandzic, M. Käske, G. Sommerkorn, and R. S. Thomä, "Large scale parameter for the WINNER II channel model at $2.53 \mathrm{GHz}$ in urban macro cell," in 2010 IEEE 71st Vehicular Technology Conference, Taipei, Taiwan, 2010.

[196] S. Jaeckel, L. Raschkowski, K. Börner, and L. Thiele, "QuaDRiGa - quasi deterministic radio channel generator, user manual and documentation," V1.2.3-307, Fraunhofer Heinrich Hertz Institute, Berlin, Germany, 2014.

[197] F. Burkhardt, S. Jaeckel, E. Eberlein, and R. Prieto-Cerdeira, "QuaDRiGa: a MIMO channel model for land mobile satellite," in The 8th European Conference on Antennas and Propagation (EuCAP 2014), pp. 1274-1278, The Hague, Netherlands, April 2014

[198] Z. Fu, X. Zhao, S. Geng et al., "Channel simulation and validation by QuaDRiGa for suburban microcells under 6 GHz," in 2018 12th International Symposium on Antennas, Propagation and EM Theory (ISAPE), pp. 2018-2021, Hangzhou, China, Decemeber 2018.

[199] B. Mondal, T. Thomas, E. Visotsky et al., "3D channel model in 3GPP," IEEE Communications Magazine, vol. 53, no. 3, pp. 16-23, 2015.

[200] V. S. Abhayawardhana, I. J. Wassellt, D. Crosby, M. P. Sellars, and M. G. Brown, "Comparison of empirical propagation path loss models for fixed wireless access systems," in 2005 IEEE 61st Vehicular Technology Conference, vol. 61no. 1, pp. 73-77, Stockholm, Sweden, 2005.
[201] V. Erceg, L. Schumacher, and P. Kyritsi, "IEEE 802.11 document 03/940r4 (TGN channel models)," 2004.

[202] L. Schumacher and B. Dijkstra, "Description of a MATLAB implementation of the indoor MIMO WLAN channel model proposed by the IEEE 802.11 TGn channel model special committee," Implementation note version 3.1, FUNDP. The University of Namur Computer Science Institute, Namur, Belgium, 2004.

[203] D. Pareit, B. Lannoo, I. Moerman, and P. Demeester, "The history of WiMAX: a complete survey of the evolution in certification and standardization for IEEE 802.16 and WiMAX," IEEE Communication Surveys and Tutorials, vol. 14, no. 4, pp. 1183-1211, 2012.

[204] A. Ghosh, D. R. Wolter, J. G. Andrews, and R. Chen, "Broadband wireless access with WiMax/802.16: current performance benchmarks and future potential," IEEE Communications Magazine, vol. 43, no. 2, pp. 129-136, 2005.

[205] S. J. Vaughan-Nichols, "Achieving wireless broadband with WiMax,” Computer, vol. 37, no. 6, pp. 10-13, 2004.

[206] D. Niyato, E. Hossain, and J. Diamond, "IEEE 802.16/WiMAX-based broadband wireless access and its application for telemedicine/E-health services," IEEE Wireless Communications, vol. 14, no. 1, pp. 72-83, 2007.

[207] C. X. Wang, F. Haider, X. Gao et al., "Cellular architecture and key technologies for $5 \mathrm{G}$ wireless communication networks," IEEE Communications Magazine, vol. 52, no. 2, pp. 122-130, 2014.

[208] X. Hong, J. Wang, C. X. Wang, and J. Shi, "Cognitive radio in 5G: a perspective on energy-spectral efficiency trade-off," IEEE Communications Magazine, vol. 52, no. 7, pp. 46-53, 2014.

[209] R. Almesaeed, A. S. Ameen, A. Doufexi, N. Dahnoun, and A. R. Nix, "A comparison study of 2D and 3D ITU channel model," in 2013 IFIP Wireless Days (WD), Valencia, Spain, November 2013.

[210] L. Hentilä, P. Kyösti, and J. Meinilä, “Elevation extension for a geometry-based radio channel model and its influence on MIMO antenna correlation and gain imbalance," in Proceedings of the 5th European Conference on Antennas and Propagation (EUCAP), pp. 2175-2179, Rome, Italy, 2011.

[211] Y. Yu, J. Zhang, M. Shafi, M. Zhang, and J. Mirza, "Statistical characteristics of measured 3-dimensional MIMO channel for outdoor-to-indoor scenario in China and New Zealand," Chinese Journal of Engineering, vol. 2016, 10 pages, 2016.

[212] T. A. Thomas, F. W. Vook, E. Visotsky, E. Mellios, G. S. Hilton, and A. R. Nix, "3D extension of the 3GPP/ITU channel model," in 2013 IEEE 77th Vehicular Technology Conference (VTC Spring), Dresden, Germany, June 2013.

[213] 3rd Generation Partnership Project, "Study on 3D channel model for LTE (Release 12) 3GPP TR 36.873 V12.1.0," 2015.

[214] 3GPP, "Draft report of 3GPP TSG RAN WG1 \#84bis v0.1.0," 2016.

[215] H. Tataria, K. Haneda, A. F. Molisch, M. Shafi, and F. Tufvesson, "Standardization of propagation models: 800 $\mathrm{MHz}$ to $100 \mathrm{GHz}$ - a historical perspective," 2020, http:// arxiv.org/abs/2006.08491.

[216] M. Jiang, M. Hosseinian, M. Il Lee, and J. Stern-Berkowitz, "3D channel model extensions and characteristics study for future wireless systems," in 2013 IEEE 24th Annual International Symposium on Personal, Indoor, and Mobile Radio 
Communications (PIMRC), pp. 41-46, London, UK, September 2013 .

[217] A. Kammoun, H. Khanfir, Z. Altman, M. Debbah, and M. Kamoun, "Preliminary results on 3D channel modeling: from theory to standardization," IEEE Journal on Selected Areas in Communications, vol. 32, no. 6, pp. 1219-1229, 2014.

[218] F. Ademaj, M. Taranetz, and M. Rupp, "Implementation, validation and application of the 3GPP 3D MIMO channel model in open source simulation tools," in 2015 International Symposium on Wireless Communication Systems (ISWCS), pp. 721-725, Brussels, Belgium, August 2015.

[219] F. Ademaj, M. Taranetz, and M. Rupp, “3GPP 3D MIMO channel model: a holistic implementation guideline for open source simulation tools," EURASIP Journal on Wireless Communications and Networking, vol. 2016, no. 1, Article ID 55, p. 14, 2016.

[220] E. Basar and I. Yildirim, "Indoor and outdoor physical channel modeling and efficient positioning for reconfigurable intelligent surfaces in mmWave bands," pp. 1-10, 2020, http://arxiv.org/abs/2006.02240.

[221] D. Serghiou, M. Khalily, S. Johny et al., "Ultra-wideband Terahertz channel propagation measurements from 500 to 750 $\mathrm{GHz}$," in 2020 International Conference on UK-China Emerging Technologies (UCET), pp. 1-4, Glasgow, United Kingdom, August 2020.

[222] A. R. Ekti, A. Boyaci, A. Alparslan et al., "Statistical modeling of propagation channels for Terahertz band," in 2017 IEEE Conference on Standards for Communications and Networking (CSCN), pp. 275-280, Helsinki, Finland, September 2017.

[223] S. Yong, IEEE 802.15.3c TG3c Channel Modeling Subcommittee Report Final Report, Institute of Electrical and Electronics Engineers (IEEE), 2006.

[224] E. Perahia, C. Cordeiro, M. Park, and L. L. Yang, "IEEE 802.11ad: defining the next generation multi-Gbps Wi-Fi," in 2010 7th IEEE Consumer Communications and Networking Conference, Las Vegas, NV, USA, January 2010.

[225] A. L. Imoize, Analysis of Propagation Models for Mobile Radio Reception at $1800 \mathrm{MHz}$, University of Lagos, Nigeria, 2011.

[226] Z. Hu, R. Liu, S. Kang, X. Su, and J. Xu, "Work in progress: 3D beamforming methods with user-specific elevation beamfoming," in 9th International Conference on Communications and Networking in China, pp. 383-386, Maoming, China, August 2014.

[227] Q. Hong, J. Zhang, H. Zheng et al., "The impact of antenna height on 3D channel: a ray launching based analysis," Electronics (Basel), vol. 7, no. 1, p. 2, 2018.

[228] J. Zhang, C. Pan, F. Pei, G. Liu, and X. Cheng, “Three-dimensional fading channel models: a survey of elevation angle research," IEEE Communications Magazine, vol. 52, no. 6, pp. 218-226, 2014.

[229] T. A. Thomas, H. C. Nguyen, G. R. Maccartney, and T. S. Rappaport, "3D mmWave channel model proposal," in 2014 IEEE 80th Vehicular Technology Conference (VTC2014-Fall), Vancouver, BC, Canada, September 2014.

[230] I. Sen and D. W. Matolak, "Vehicle-vehicle channel models for the 5-GHz band," IEEE Transactions on Intelligent Transportation Systems, vol. 9, no. 2, pp. 235-245, 2008.

[231] C. X. Wang, X. Cheng, and D. Laurenson, "Vehicle-to-vehicle channel modeling and measurements: recent advances and future challenges," IEEE Communications Magazine, vol. 47, no. 11, pp. 96-103, 2009.

[232] A. Chelli and M. Pätzold, "A non-stationary MIMO vehicleto-vehicle channel model based on the geometrical Tjunction model," in 2009 International Conference on Wireless Communications \& Signal Processing, Nanjing, China, November 2009.

[233] A. F. Molisch, F. Tufvesson, J. Karedal, and C. Mecklenbrauker, "A survey on vehicle-to-vehicle propagation channels," IEEE Wireless Communications, vol. 16, no. 6, pp. 12-22, 2009.

[234] A. Chelli and M. Pätzold, "A non-stationary MIMO vehicleto-vehicle channel model derived from the geometrical street model," in 2011 IEEE Vehicular Technology Conference (VTC Fall), San Francisco, CA, USA, September 2011.

[235] J. Karedal, N. Czink, A. Paier, F. Tufvesson, and A. F. Molisch, "Path loss modeling for vehicle-to-vehicle communications," IEEE Transactions on Vehicular Technology, vol. 60 , no. 1, pp. 323-328, 2011.

[236] G. Acosta, K. Tokuda, and M. A. Ingram, "Measured joint doppler-delay power profiles for vehicle-to-vehicle communications at $2.4 \mathrm{GHz}$," in IEEE Global Telecommunications Conference, 2004. GLOBECOM '04, pp. 3813-3817, Dallas, TX, USA, 2004.

[237] D. W. Matolak, "Channel modeling for vehicle-to-vehicle communications," IEEE Communications Magazine, vol. 46, no. 5, pp. 76-83, 2008.

[238] D. Solomitckii, Y. Koucheryavy, V. Semkin et al., "Characterizing radio wave propagation in urban street canyon with vehicular blockage at $28 \mathrm{GHz}$," IEEE Transactions on Vehicular Technology, vol. 69, no. 2, pp. 1227-1236, 2020.

[239] L. Wu, D. He, B. Ai et al., "Artificial neural network based path loss prediction for wireless communication network," IEEE Access, vol. 8, pp. 199523-199538, 2020.

[240] V. Petrov, J. M. Eckhardt, D. Moltchanov, Y. Koucheryavy, and T. Kurner, "Measurements of reflection and penetration losses in low terahertz band vehicular communications," in 2020 14th European Conference on Antennas and Propagation (EuCAP), Copenhagen, Denmark, March 2020. 